\title{
SAPHIRE 8 Quality Assurance \\ Software Metrics Report
}

Kurt G. Vedros

August 2011
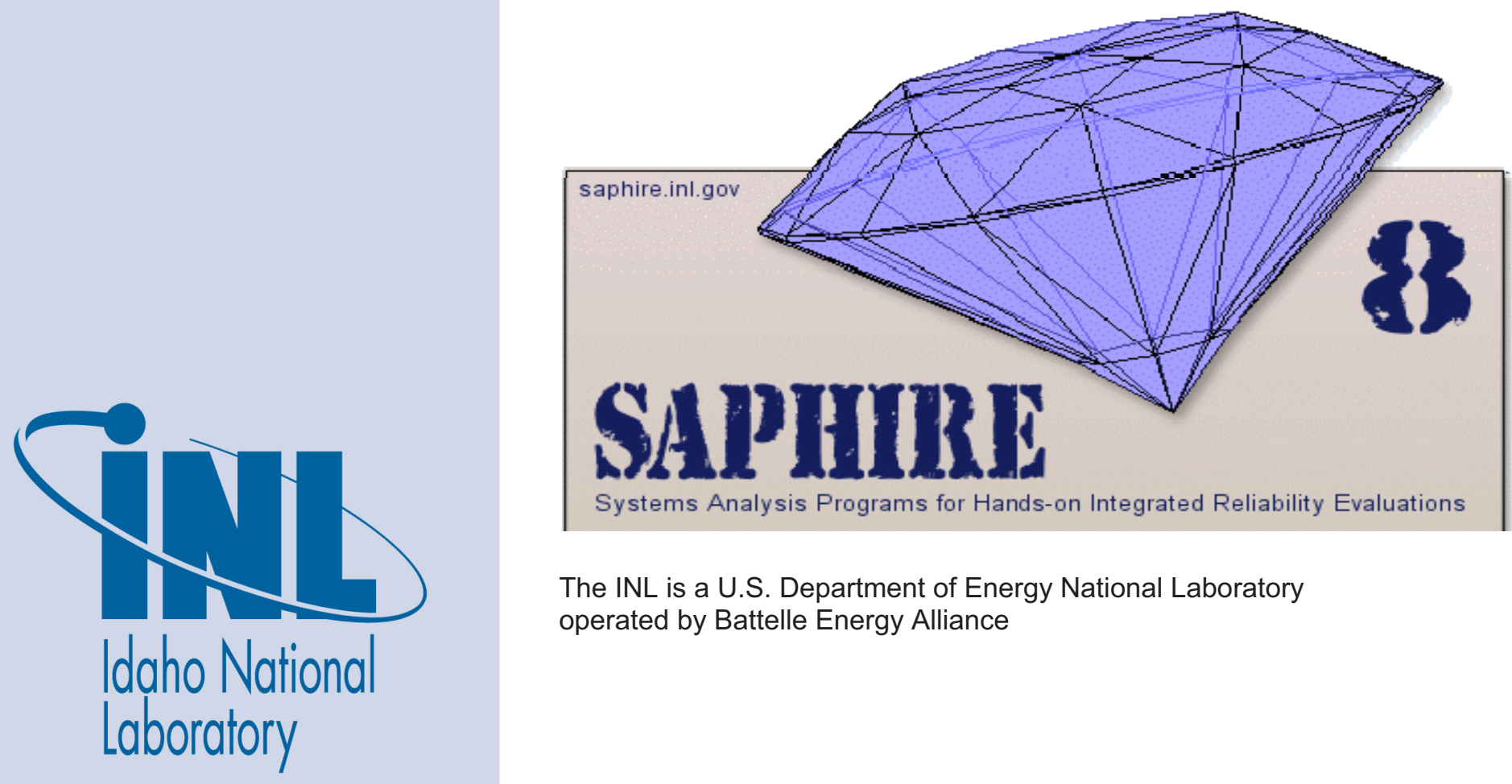

The INL is a U.S. Department of Energy National Laboratory operated by Battelle Energy Alliance 
INL/EXT-11-23138

\title{
SAPHIRE 8 Quality Assurance Software Metrics Report
}

\author{
Kurt G. Vedros
}

August 2011

Idaho National Laboratory

Idaho Falls, Idaho 83415

http://www.inl.gov

Prepared for the

U.S. Nuclear Regulatory Commission

Washington, DC 20555

Project Number V6059 


\section{Table of Contents}

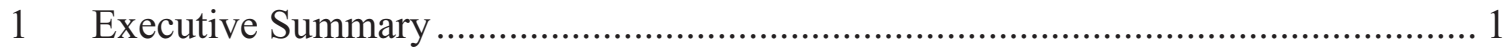

2 Background Information ................................................................................. 2

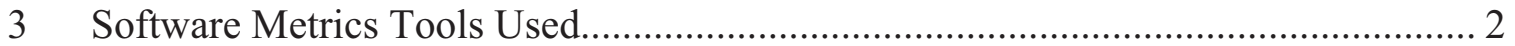

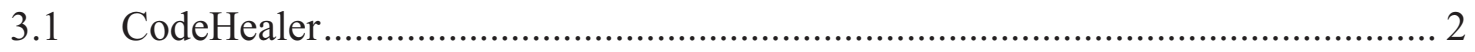

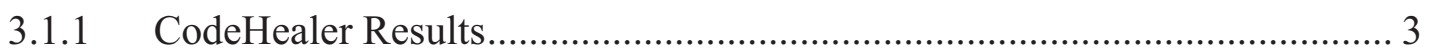

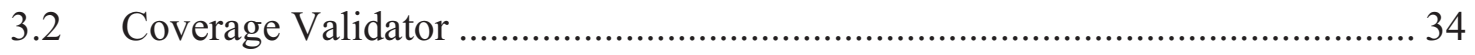

3.2.1 Coverage Validator Results ................................................................ 34

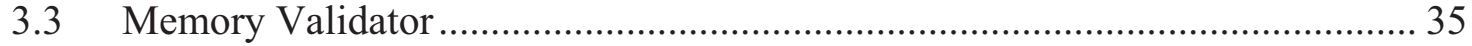

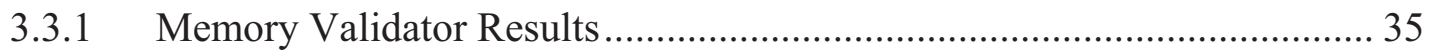

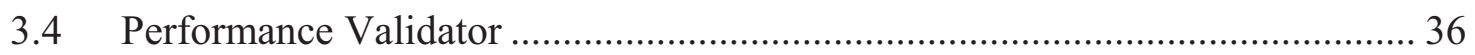

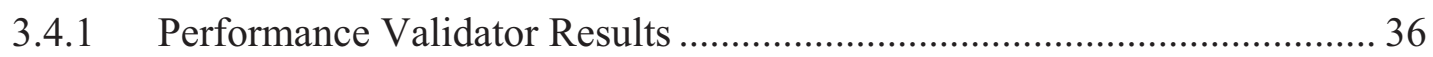

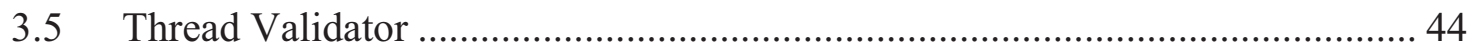

3.5.1 Thread Validator Results ....................................................................... 44

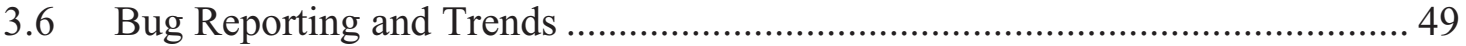

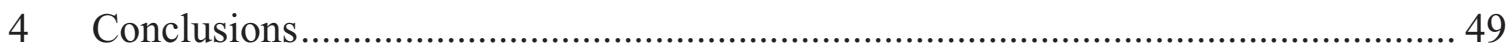

\section{Table of Figures}

Figure 3-1 Source Code Analysis for CodeHealer SAPHIRE 8 v8.06.01 ....................... 31

Figure 3-2 Audits Category for CodeHealer Project SAPHIRE 8 Analysis..................... 32

Figure 3-3 Checks Category Settings for CodeHealer Project SAPHIRE 8 Analysis..... 33

Figure 3-4 Memory Validator Memory Leak and Handle Leak Detection v8.06.01 ...... 36

Figure 3-5 Memory Validator Memory Leak and Handle Leak Detection v8.07.17 ..... 36

Figure 3-6 SAPHIRE 8 v8.06.01 Performance Validator SDP Statistics Report - Left.. 38

Figure 3-7 SAPHIRE 8 v8.06.01 Performance Validator SDP Statistics Report - Right 39

Figure 3-8 SAPHIRE 9 v8.06.01 Performance Validator ECA Statistics Report - Left . 40

Figure 3-9 SAPHIRE 8 v8.06.01 Performance Validator ECA Statistics Report - Right41

Figure 3-10 SAPHIRE 8 v8.06.01 Performance Validator General Analysis Statistics

Report - Left. 
Figure 3-11 SAPHIRE 8 v8.06.01 Performance Validator General Analysis Statistics

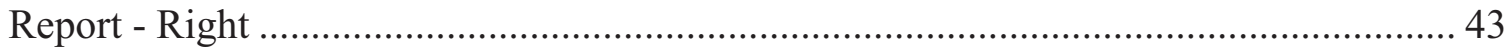

Figure 3-12 Thread Validator Lock Colors Definitions ................................................ 45

Figure 3-13 SAPHIRE 8 v8.06.01Thread Validator SDP Critical Sections and Waits

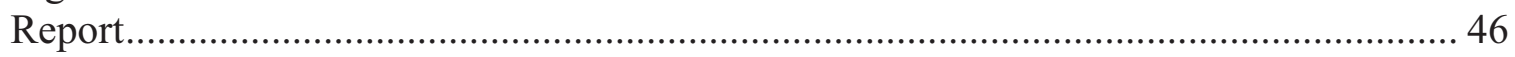

Figure 3-14 SAPHIRE 8 v8.06.01 Thread Validator ECA Critical Sections and Waits

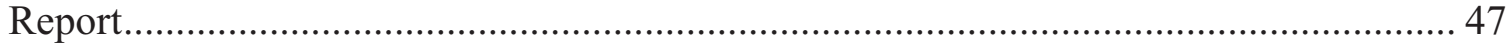

Figure 3-15 SAPHIRE 8 v8.06.01 Thread Validator General Analysis Critical Sections

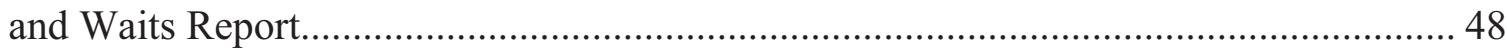




\section{Executive Summary}

The purpose of this report of the software metrics review is to review the results of the 2010 IV\&V and to set an outline for results of updated metrics runs to be performed going forward.

The purpose of the software metrics review is to show maintenance of accepted quality standards presented in SAPHIRE 8's Independent Verification and Validation (IV\&V) of April, 2010. Recommendations from the final IV\&V were to continue periodic measurable metrics such as McCabe's complexity measure to ensure quality is maintained. The four software tools used to measure quality in the IV\&V were CodeHealer, Coverage Validator, Memory Validator, Performance Validator, and Thread Validator. These will be re-run with the latest revision of SAPHIRE 8 for results comparison. 


\section{Background Information}

NUREG/BR-0167, Software Quality Assurance Program and Guidelines notes that "no metrics to support an IEEE-1012 classification higher than a '1' currently exists"; however, Section 5.2.4 requires that "metrics be part of the Tracking and Oversight function". The metrics chosen for quality in the IV\&V were from the software tools CodeHealer, Coverage Validator, Memory Validator, Performance Validator, and Thread Validator.

The IV\&V team suggested a couple of metrics that could be implemented to further track and approve quality. A metric considered at the time of the final report was an "error tracking" metric which was the errors discovered by IV\&V while evaluating the Significance Determination Process (SDP). This potentially could be compared to other development module error tracking. This would require the development team to track code errors for comparison. Bug resolution tracking was suggested as well. It was suggested that a metric of a Pareto diagram of the bug resolutions could prove useful.

\section{Software Metrics Tools Used}

\subsection{CodeHealer}

Table 7-1 provides an overview of the basic constructs used in the SAPHIRE version 8 source code using the source code analysis and verification tool CodeHealer for Delphi (C) Version 2.5.0. Any and all extra characters after the terminating "end." of a program, unit or project file are ignored, and any extra lines are always treated as blank lines, regardless of what they contain. It is possible for the total number of lines to be greater than the sum of the number of blank lines, the number of code lines and the number of comment lines if one or more lines contain a number of spaces or tab characters and nothing else, since these lines do not fit in any of the counted categories.

The majority of the metrics values are self explanatory from their name, such as the number of characters, lines, units or other basic item, so are not defined further here. Certain metrics values, however, justify further explanation, and these are listed below.

Note that because the metric values are displayed on a module basis, the Branches, Loops and Cyclomatic complexity metrics values are calculated and shown as an average of the values for each function, procedure and (class) method in the module, rather than as a total for the module.

Branches - This metric value is the average number of branches per function or procedure that occur within the source code of the selected items. A branch in the source code is an "if", "goto", "try .. except" statement or a case item or "else" value of a "case" statement, and represents a point where the flow of control may take one of two alternative routes. The number of branches within a function, procedure or module is (historically) considered as a very basic indicator of its complexity.

Loops - This metric value is the total number of loops that occur within the source code of the selected items. A loop in the source code is a "for", "repeat" or "while" statement, and represents a point where the flow of control may take one of two alternative routes on more than one occasion. The number of loops within a function, procedure or module is (historically) considered as a very basic indicator of its complexity. 
Cyclomatic Complexity - This metric value was introduced by Thomas McCabe in 1976, and it measures the number of possible paths through a source code item. It is widely considered a broad measure of soundness and confidence for a program, and also gives an indication of the minimum number of test cases that are necessary to properly test each item of the program. The cyclomatic complexity of a module is calculated as the average of the cyclomatic complexity values of each function, procedure and (class) method of the module. Table 7-2 provides the individual SAPHIRE Version 8 source code files Cyclomatic Complexity. The cyclomatic complexity of a function, procedure or (class) method is calculated as:

$1+$ Branches + Loops + Number of logical "and" and "or" operations within conditional expressions where Branches and Loops are calculated as defined above.

The Carnegie Mellon Software Engineering Institute definition of Cyclomatic Complexity can be viewed at "CMU - Cyclomatic Complexity", and part of that definition is the following suggested set of threshold values that may be used to assess the risk associated with modifying a module:

Complexity $\quad$ Risk evaluation for the module or program

$1-10 \quad$ Relatively simple, without much risk

$11-20 \quad$ More complex, moderate risk

$21-50 \quad$ Complex, high risk

Greater than $50 \quad$ Untestable (very high risk)

\subsubsection{CodeHealer Results}

Table 3-1 Source Code Metrics for CodeHealer Project SAPHIRE 8

\begin{tabular}{|c|c|c|}
\hline Metric Value & Total v8.06.01 & Total v8.07.17 \\
\hline Source Files & 1,291 & \\
\hline Programs & 6 & \\
\hline Libraries & 12 & \\
\hline Packages & 1 & \\
\hline Units & 1,272 & \\
\hline Forms & 405 & \\
\hline Main Used Units & 1,428 & \\
\hline Used Units (not unique) & 20,981 & \\
\hline Characters & $53,035,946$ & \\
\hline Lines & $1,846,865$ & \\
\hline Blank Lines & 371,863 & \\
\hline Branches (average) & 2 & \\
\hline
\end{tabular}




\begin{tabular}{|c|c|c|}
\hline Metric Value & Total v8.06.01 & Total v8.07.17 \\
\hline Loops (average) & 1 & \\
\hline Cyclomatic Complexity (average) & 4 & \\
\hline Commented Code Lines & 41,869 & \\
\hline Pure Code Lines & $1,182,202$ & \\
\hline Code Lines & $1,224,071$ & \\
\hline Significant Comment Lines & 242,243 & \\
\hline Blank Comment Lines & 7,711 & \\
\hline Comment Lines & 249,954 & \\
\hline Total Comments (\%) & $19 \%$ & \\
\hline Significant Comments (\%) & $19 \%$ & \\
\hline Classes & 1,775 & \\
\hline Interfaces & 27 & \\
\hline Objects & 0 & \\
\hline Properties & 5,346 & \\
\hline Constructors & 1,295 & \\
\hline Destructors & 736 & \\
\hline Functions & 12,439 & \\
\hline Procedures & 32,047 & \\
\hline Initializations & 84 & \\
\hline Finalizations & 35 & \\
\hline Keywords & 796,973 & \\
\hline Directives & 65,281 & \\
\hline Identifiers & $2,168,392$ & \\
\hline Hex Integers & 8,665 & \\
\hline Integers & 174,790 & \\
\hline Labels & 2 & \\
\hline Reals & 11,735 & \\
\hline String Constants & 75,985 & \\
\hline
\end{tabular}


Table 3-2 Individual SAPHIRE 8 Source Code File Cyclomatic Complexity

\begin{tabular}{|c|c|c|c|}
\hline \multirow[t]{2}{*}{ File Name } & \multirow[t]{2}{*}{ File Path } & \multicolumn{2}{|c|}{$\begin{array}{l}\text { Cyclomatic } \\
\text { Complexity }\end{array}$} \\
\hline & & v8.06.01 & v8.07.17 \\
\hline AboutUnit & C: $\mid$ Saphire 8\Debug $\backslash$ source $\backslash$ schemaObjects $\backslash$ & 1 & \\
\hline AdjListPanel & C: \Saphire $8 \backslash$ Debug $\backslash$ source $\backslash$ DelphiSource $\backslash$ & 6 & \\
\hline AnalysisPointETObject.pas & $\begin{array}{l}\text { C: } \backslash \text { Saphire } \\
\text { 8\Debug } \backslash \text { source } \backslash \text { DelphiSource } \backslash \text { GraphicalEditi } \\
\text { ng } \backslash\end{array}$ & 4 & \\
\hline AnalysisPointFTCSPathObject.pas & $\begin{array}{l}\text { C: } \mid \text { Saphire } \\
8 \backslash \text { Debug } \mid \text { source } \backslash \text { DelphiSourcelGraphicalEditi } \\
\text { ng } \backslash\end{array}$ & 7 & \\
\hline AnalysisPointFTObject.pas & $\begin{array}{l}\text { C: } \backslash \text { Saphire } \\
\text { 8\Debug } \backslash \text { source } \backslash \text { DelphiSource } \backslash \text { GraphicalEditi } \\
\text { ng } \backslash\end{array}$ & 6 & \\
\hline AnalysisPointObject.pas & $\begin{array}{l}\text { C: } \backslash \text { Saphire } \\
8 \backslash \text { Debug } \backslash \text { source } \backslash \text { DelphiSource } \backslash \text { GraphicalEditi } \\
\text { ng } \backslash\end{array}$ & 2 & \\
\hline AnalyzeGeneral.pas & C:|Saphire 8\Debug \source $\backslash$ DelphiSource $\backslash$ & 3 & \\
\hline AnalyzeSDP.pas & C:|Saphire 8\Debug $\mid$ source $\backslash$ DelphiSource $\backslash$ & 3 & \\
\hline API_AnalysisType.pas & $\begin{array}{l}\text { C: } \backslash \text { Saphire } \\
\text { 8\Debug } \backslash \text { source } \backslash \text { DelphiSource } \backslash \text { RiskApi } \backslash\end{array}$ & 2 & \\
\hline API_Assess.pas & C:|Saphire 8\Debug $\mid$ source $\mid$ DelphiSource $\backslash$ & 0 & \\
\hline API_Assess.pas & $\begin{array}{l}\text { C: } \backslash \text { Saphire } \\
\text { 8\Debug } \backslash \text { source } \backslash \text { DelphiSource } \backslash \text { RiskApi } \backslash\end{array}$ & 1 & \\
\hline API_BlockDiagram.pas & $\begin{array}{l}\text { C: } \backslash \text { Saphire } \\
8 \backslash \text { Debug } \backslash \text { source } \backslash \text { DelphiSource } \backslash \text { RiskApi } \backslash\end{array}$ & 0 & \\
\hline API_Cat.pas & $\begin{array}{l}\text { C: } \backslash \text { Saphire } \\
8 \backslash \text { Debug } \backslash \text { source } \backslash \text { DelphiSource } \backslash \text { RiskApi } \backslash\end{array}$ & 1 & \\
\hline API_Category.pas & $\begin{array}{l}\text { C: } \backslash \text { Saphire } \\
8 \backslash \text { Debug } \backslash \text { source } \backslash \text { DelphiSource } \backslash \text { RiskApi } \backslash\end{array}$ & 1 & \\
\hline API_ChgSet.pas & $\begin{array}{l}\text { C: } \backslash \text { Saphire } \\
8 \backslash \text { Debug } \backslash \text { source } \backslash \text { DelphiSource } \backslash \text { RiskApi } \backslash\end{array}$ & 2 & \\
\hline API_CompareProject.pas & 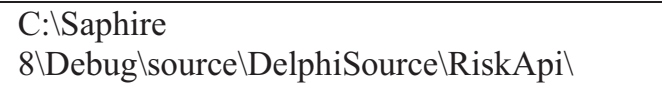 & 1 & \\
\hline API_Constants.pas & $\begin{array}{l}\text { C: } \backslash \text { Saphire } \\
8 \backslash \text { Debug } \backslash \text { source } \backslash \text { DelphiSource } \backslash \text { RiskApi } \backslash\end{array}$ & 1 & \\
\hline API_DefSolve.pas & $\begin{array}{l}\text { C: \Saphire } \\
\text { 8\Debug \source \DelphiSource\RiskApi }\end{array}$ & 1 & \\
\hline API_EndState.pas & $\begin{array}{l}\text { C: } \backslash \text { Saphire } \\
8 \backslash \text { Debug } \backslash \text { source } \backslash \text { DelphiSource } \backslash \text { RiskApi } \backslash\end{array}$ & 1 & \\
\hline API_ESCat.pas & $\begin{array}{l}\text { C: } \backslash \text { Saphire } \\
\text { 8\Debug } \backslash \text { source } \backslash \text { DelphiSource } \backslash \text { RiskApi } \backslash\end{array}$ & 1 & \\
\hline API_EvCat.pas & $\begin{array}{l}\text { C: } \backslash \text { Saphire } \\
\text { 8\Debug } \backslash \text { source } \backslash \text { DelphiSource } \backslash \text { RiskApi } \backslash\end{array}$ & 1 & \\
\hline
\end{tabular}




\begin{tabular}{|c|c|c|c|}
\hline \multirow[t]{2}{*}{ File Name } & \multirow[t]{2}{*}{ File Path } & \multicolumn{2}{|c|}{$\begin{array}{l}\text { Cyclomatic } \\
\text { Complexity }\end{array}$} \\
\hline & & v8.06.01 & v8.07.17 \\
\hline API_EvCmpLib.pas & $\begin{array}{l}\text { C: } \backslash \text { Saphire } \\
\text { 8\Debug } \backslash \text { source } \backslash \text { DelphiSource } \backslash \text { RiskApi } \backslash\end{array}$ & 2 & \\
\hline API_Event.pas & C:|Saphire 8\Debug \source\DelphiSource $\backslash$ & 0 & \\
\hline API_Event.pas & $\begin{array}{l}\text { C: } \backslash \text { Saphire } \\
\text { 8\Debug } \backslash \text { source } \backslash \text { DelphiSource } \backslash \text { RiskApi } \backslash\end{array}$ & 2 & \\
\hline API_EventSet.pas & $\begin{array}{l}\text { C: } \backslash \text { Saphire } \\
8 \backslash \text { Debug } \backslash \text { source } \backslash \text { DelphiSource } \backslash \text { RiskApi } \backslash\end{array}$ & 1 & \\
\hline API_EventTree.pas & C:|Saphire 8\Debug\source\DelphiSource $\backslash$ & 0 & \\
\hline API_EventTree.pas & $\begin{array}{l}\text { C: } \backslash \text { Saphire } \\
8 \backslash \text { Debug } \backslash \text { source } \backslash \text { DelphiSource } \backslash \text { RiskApi } \backslash\end{array}$ & 2 & \\
\hline API_Extract.pas & $\begin{array}{l}\text { C:ISaphire } \\
8 \backslash \text { Debug } \backslash \text { source } \backslash \text { DelphiSource } \backslash \text { RiskApi } \backslash\end{array}$ & 2 & \\
\hline API_Gate.pas & $\begin{array}{l}\text { C: } \backslash \text { Saphire } \\
8 \backslash \text { Debug } \backslash \text { source } \backslash \text { DelphiSource } \backslash \text { RiskApi } \backslash\end{array}$ & 1 & \\
\hline API_Gather.pas & $\begin{array}{l}\text { C: } \mid \text { Saphire } \\
\text { 8\Debug } \backslash \text { source } \backslash \text { DelphiSource } \backslash \text { RiskApi } \backslash\end{array}$ & 1 & \\
\hline API_Generate.pas & $\begin{array}{l}\text { C: } \mid \text { Saphire } \\
8 \backslash \text { Debug } \backslash \text { source } \backslash \text { DelphiSource } \backslash \text { RiskApi } \backslash\end{array}$ & 1 & \\
\hline API_Histogram.pas & $\begin{array}{l}\text { C: } \backslash \text { Saphire } \\
8 \backslash \text { Debug } \backslash \text { source } \backslash \text { DelphiSource } \backslash \text { RiskApi } \backslash\end{array}$ & 1 & \\
\hline API_Importance.pas & $\begin{array}{l}\text { C: } \backslash \text { Saphire } \\
8 \backslash \text { Debug } \backslash \text { source } \backslash \text { DelphiSource } \backslash \text { RiskApi } \backslash\end{array}$ & 0 & \\
\hline API_Load.pas & $\begin{array}{l}\text { C: } \backslash \text { Saphire } \\
8 \backslash \text { Debug } \backslash \text { source } \backslash \text { DelphiSource } \backslash \text { RiskApi } \backslash\end{array}$ & 1 & \\
\hline API_Logic.pas & $\begin{array}{l}\text { C: } \mid \text { Saphire } \\
8 \backslash \text { Debug } \backslash \text { source } \backslash \text { DelphiSource } \backslash \text { RiskApi } \backslash\end{array}$ & 1 & \\
\hline API_MergeProjects.pas & $\begin{array}{l}\text { C: } \backslash \text { Saphire } \\
\text { 8\Debug } \backslash \text { source } \backslash \text { DelphiSource } \backslash \text { RiskApi } \backslash\end{array}$ & 1 & \\
\hline API_Partition.pas & $\begin{array}{l}\text { C: } \mid \text { Saphire } \\
\text { 8\Debug } \mid \text { source } \backslash \text { DelphiSource } \backslash \text { RiskApi } \backslash\end{array}$ & 0 & \\
\hline API_Phase.pas & $\begin{array}{l}\text { C: } \backslash \text { Saphire } \\
8 \backslash \text { Debug } \backslash \text { source } \backslash \text { DelphiSource } \backslash \text { RiskApi } \backslash\end{array}$ & 1 & \\
\hline API_Project.pas & $\begin{array}{l}\text { C: } \mid \text { Saphire } \\
\text { 8\Debug } \backslash \text { source } \backslash \text { DelphiSource } \backslash \text { RiskApi } \backslash\end{array}$ & 2 & \\
\hline API_Quantify.pas & $\begin{array}{l}\text { C: } \mid \text { Saphire } \\
\text { 8\Debug } \backslash \text { source } \backslash \text { DelphiSource } \backslash \text { RiskApi } \backslash\end{array}$ & 0 & \\
\hline API_RaspCCF.pas & $\begin{array}{l}\text { C: } \mid \text { Saphire } \\
\text { 8\Debug|source } \backslash \text { DelphiSource } \backslash \text { RiskApi }\end{array}$ & 1 & \\
\hline API_Recover.pas & $\begin{array}{l}\text { C:|Saphire } \\
\text { 8\Debug|source } \backslash \text { DelphiSource } \backslash \text { RiskApi } \backslash\end{array}$ & 0 & \\
\hline API_ReportCust.pas & $\begin{array}{l}\text { C: } \backslash \text { Saphire } \\
\text { 8\Debug } \backslash \text { source } \backslash \text { DelphiSource } \backslash \text { RiskApi } \backslash\end{array}$ & 1 & \\
\hline API_Reports7.pas & C:ISaphire & 4 & \\
\hline
\end{tabular}




\begin{tabular}{|c|c|c|c|}
\hline \multirow[t]{2}{*}{ File Name } & \multirow[t]{2}{*}{ File Path } & \multicolumn{2}{|c|}{$\begin{array}{l}\text { Cyclomatic } \\
\text { Complexity }\end{array}$} \\
\hline & & v8.06.01 & v8.07.17 \\
\hline & $8 \backslash$ Debug $\backslash$ source $\backslash$ DelphiSource\RiskApi & & \\
\hline API_Risk.pas & $\begin{array}{l}\text { C: } \backslash \text { Saphire } \\
\text { 8\Debug } \backslash \text { source } \backslash \text { DelphiSource } \backslash \text { RiskApi } \backslash\end{array}$ & 2 & \\
\hline API_RiskErr.pas & $\begin{array}{l}\text { C: } \backslash \text { Saphire } \\
8 \backslash \text { Debug } \backslash \text { source } \backslash \text { DelphiSource\RiskApi } \backslash\end{array}$ & 0 & \\
\hline API_RiskMacro.pas & $\begin{array}{l}\text { C: } \backslash \text { Saphire } \\
\text { 8\Debug } \backslash \text { source } \backslash \text { DelphiSource } \backslash \text { RiskApi } \backslash\end{array}$ & 1 & \\
\hline API_RulesCode.pas & $\begin{array}{l}\text { C: } \backslash \text { Saphire } \\
\text { 8\Debug } \backslash \text { source } \backslash \text { DelphiSource } \backslash \text { RiskApi }\end{array}$ & 2 & \\
\hline API_Sequence.pas & C: $\mid$ Saphire $8 \backslash$ Debug $\backslash$ source $\backslash$ DelphiSource $\backslash$ & 0 & \\
\hline API_Sequence.pas & $\begin{array}{l}\text { C:ISaphire } \\
8 \backslash \text { Debug } \backslash \text { source } \backslash \text { DelphiSource } \backslash \text { RiskApi } \backslash\end{array}$ & 1 & \\
\hline API_Simulation.pas & $\begin{array}{l}\text { C: } \backslash \text { Saphire } \\
\text { 8\Debug } \backslash \text { source } \backslash \text { DelphiSource\RiskApi } \backslash\end{array}$ & 1 & \\
\hline API_Slice.pas & $\begin{array}{l}\text { C: } \backslash \text { Saphire } \\
\text { 8\Debug } \backslash \text { source } \backslash \text { DelphiSource } \backslash R i s k A p i \backslash\end{array}$ & 1 & \\
\hline API_System.pas & $\begin{array}{l}\text { C: } \backslash \text { Saphire } \\
8 \backslash \text { Debug } \backslash \text { source } \backslash \text { DelphiSource } \backslash \text { RiskApi } \backslash\end{array}$ & 2 & \\
\hline API_TreeRead.pas & $\begin{array}{l}\text { C: } \backslash \text { Saphire } \\
\text { 8\Debug } \backslash \text { source } \backslash \text { DelphiSource } \backslash \text { RiskApi } \backslash\end{array}$ & 1 & \\
\hline API_Uncertainty.pas & $\begin{array}{l}\text { C:|Saphire } \\
\text { 8\Debug|source } \backslash \text { DelphiSource } \backslash \text { RiskApi } \backslash\end{array}$ & 1 & \\
\hline API_Update.pas & $\begin{array}{l}\text { C: } \backslash \text { Saphire } \\
\text { 8\Debug } \backslash \text { source } \backslash \text { DelphiSource } \backslash \text { RiskApi }\end{array}$ & 0 & \\
\hline API_WorkSpace.pas & C:ISaphire 8\Debug \source \DelphiSource $\backslash$ & 1 & \\
\hline API_WorkSpace.pas & $\begin{array}{l}\text { C: } \backslash \text { Saphire } \\
\text { 8\Debug } \backslash \text { source } \backslash \text { DelphiSource } \backslash \text { RiskApi } \backslash\end{array}$ & 1 & \\
\hline APIX_XOverMain.pas & $\begin{array}{l}\text { C: } \backslash \text { Saphire } \\
8 \backslash \text { Debug } \backslash \text { source } \backslash \text { DelphiSource } \backslash \text { RiskApi } \backslash\end{array}$ & 3 & \\
\hline ApplicableTypes.pas & C:ISaphire 8\Debug \source\DelphiSource $\backslash$ & 3 & \\
\hline ApplicableTypes.pas & 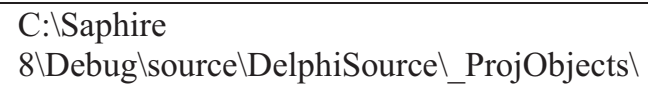 & 3 & \\
\hline ASCIIX.pas & C:|Saphire 8\Debug|source $\backslash$ & 0 & \\
\hline AscRptLib.pas & C:ISaphire 8\Debug|source\} $&{6} &{ } \\
{\hline \text { AutoFT.pas }} &{\begin{array}{l}\text { C: } \mid \text { Saphire } \\
8 \backslash \text { Debug } \backslash \text { source } \backslash \text { DelphiSource } \backslash \text { BddItems } \backslash\end{array}} &{14} &{ } \\
{\hline \text { AuxSpec.pas }} &{\text { C:ISaphire 8\Debug|source\} } &{0} &{ } \\
{\hline \text { BaseDef.pas }} &{\begin{array}{l}\text { C: } \backslash \text { Saphire } \\
8 \backslash \text { Debug } \backslash \text { source } \backslash \text { DelphiSource } \backslash \text { PlugIns } \backslash\end{array}} &{1} &{ } \\
{\hline \text { BaseDefs.pas }} &{\text { C: \Saphire 8\Debug \source\DelphiSource } \backslash} &{3} &{ } \\
{\hline \text { BasicFieldClass.pas }} &{\text { C:|Saphire 8\Debug } \mid \text { source } \backslash} &{2} &{ } \\
\hline\end{array}$
\end{tabular}




\begin{tabular}{|c|c|c|c|}
\hline \multirow[t]{2}{*}{ File Name } & \multirow[t]{2}{*}{ File Path } & \multicolumn{2}{|c|}{$\begin{array}{l}\text { Cyclomatic } \\
\text { Complexity }\end{array}$} \\
\hline & & v8.06.01 & v8.07.17 \\
\hline BasicRelationClass.pas & C:|Saphire $8 \backslash$ Debug $\mid$ source $\backslash$ & 2 & \\
\hline BasicSchemaClass.pas & C: $\mid$ Saphire $8 \backslash$ Debug $\mid$ source $\backslash$ & 2 & \\
\hline BENodeEditor.pas & C:ISaphire 8\Debug \source \DelphiSource $\backslash$ & 13 & \\
\hline BENodeEditor.pas & $\begin{array}{l}\text { C: } \backslash \text { Saphire } \\
8 \backslash \text { Debug } \backslash \text { source } \backslash \text { DelphiSource } \backslash \text { EventEditing } \backslash\end{array}$ & 5 & \\
\hline BEObject.pas & C:ISaphire 8\Debug \source\DelphiSource $\backslash$ & 4 & \\
\hline BEObject.pas & $\begin{array}{l}\text { C: } \backslash \text { Saphire } \\
\text { 8\Debug } \backslash \text { source } \backslash \text { DelphiSource } \backslash \text { ProjObjects } \backslash\end{array}$ & 4 & \\
\hline BEProbability.pas & C:ISaphire 8\Debug \source $\backslash$ DelphiSource $\backslash$ & 14 & \\
\hline BEQuick.pas & C:ISaphire 8\Debug \source $\mid$ DelphiSource $\backslash$ & 3 & \\
\hline BEQuick.pas & $\begin{array}{l}\text { C: } \backslash \text { Saphire } \\
\text { 8\Debug } \backslash \text { source \DelphiSource\_ProjObjects } \backslash\end{array}$ & 3 & \\
\hline BESelectList.pas & C: $\backslash$ Saphire $8 \backslash$ Debug $\backslash$ source $\backslash$ DelphiSource $\backslash$ & 2 & \\
\hline BinLib.pas & C: Saphire 8\Debug $\mid$ source $\backslash$ & 3 & \\
\hline BitLib.pas & C:ISaphire 8\Debug|source $\backslash$ & 2 & \\
\hline BitMapFont.pas & C:ISaphire 8\Debug|source\} $&{5} &{ } \\
{\hline \text { BitSetObj.pas }} &{\text { C:ISaphire 8\Debug \source\DelphiSource } \backslash} &{2} &{ } \\
{\hline \text { BlkEdit.pas }} &{\text { C: } \mid \text { Saphire } 8 \backslash \text { Debug } \mid \text { source } \backslash} &{3} &{ } \\
{\hline \text { BlockDataLib.pas }} &{\text { C:ISaphire 8\Debug|source\} } &{4} &{ } \\
{\hline \text { BlockIO.pas }} &{\text { C: } \mid \text { Saphire } 8 \backslash \text { Debug } \mid \text { source } \backslash} &{8} &{ } \\
{\hline \text { BMPLib.pas }} &{\text { C:ISaphire 8\Debug|source\} } &{9} &{ } \\
{\hline \text { BookmarksObj.pas }} &{\text { C:ISaphire 8\Debug \source\DelphiSource } \backslash} &{3} &{ } \\
{\hline \text { Btree.pas }} &{\text { C:|Saphire 8\Debug|source\} } &{6} &{ } \\
{\hline \text { BtreeBuf.pas }} &{\text { C: } \mid \text { Saphire } 8 \backslash \text { Debug } \mid \text { source } \backslash} &{7} &{ } \\
{\hline \text { BtrList.pas }} &{\text { C:ISaphire 8\Debug|source\} } &{4} &{ } \\
{\hline \text { BtrListClass.pas }} &{\text { C:ISaphire 8\Debug } \mid \text { source } \backslash} &{2} &{ } \\
{\hline \text { BtrStack.pas }} &{\text { C:ISaphire 8\Debug|source\} } &{4} &{ } \\
{\hline \text { BufIO.pas }} &{\text { C:ISaphire 8\Debug } \mid \text { source } \backslash} &{6} &{ } \\
{\hline \text { Cache.pas }} &{\text { C: } \mid \text { Saphire } 8 \backslash \text { Debug } \mid \text { source } \backslash} &{6} &{ } \\
{\hline \text { CatAPI.pas }} &{\text { C:ISaphire 8\Debug \source \DelphiSource } \backslash} &{0} &{ } \\
{\hline \text { CategoryObj.pas }} &{\text { C:ISaphire 8\Debug \source } \backslash \text { DelphiSource } \backslash} &{3} &{ } \\
{\hline \text { CategoryObj.pas }} &{\begin{array}{l}\text { C: } \backslash \text { Saphire } \\
\text { 8\Debug } \backslash \text { source } \backslash \text { DelphiSource } \backslash \text { ProjObjects } \backslash\end{array}} &{3} &{ } \\
{\hline \text { CatLib.pas }} &{\text { C: } \backslash \text { Saphire } 8 \backslash \text { Debug } \backslash \text { source } \backslash \text { DelphiSource } \backslash} &{3} &{ } \\
{\hline \text { CCFData.pas }} &{\text { C:ISaphire 8\Debug \source } \backslash \text { DelphiSource } \backslash} &{3} &{ } \\
{\hline \text { CCFEvLib.pas }} &{\text { C:ISaphire 8\Debug \source } \backslash \text { DelphiSource } \backslash} &{4} &{ } \\
{\hline \text { CCFRAlpha.pas }} &{\text { C:ISaphire }} &{4} &{ } \\
\hline\end{array}$
\end{tabular}




\begin{tabular}{|c|c|c|c|}
\hline \multirow[t]{2}{*}{ File Name } & \multirow[t]{2}{*}{ File Path } & \multicolumn{2}{|c|}{$\begin{array}{l}\text { Cyclomatic } \\
\text { Complexity }\end{array}$} \\
\hline & & v8.06.01 & v8.07.17 \\
\hline & $8 \backslash$ Debug $\backslash$ source $\backslash$ DelphiSource $\backslash$ RaspCCF $\backslash$ & & \\
\hline CCFRBase.pas & $\begin{array}{l}\text { C: } \backslash \text { Saphire } \\
8 \backslash \text { Debug } \backslash \text { source } \backslash \text { DelphiSource } \backslash \text { EventEditing } \backslash\end{array}$ & 4 & \\
\hline CCFRBeta.pas & $\begin{array}{l}\text { C: } \backslash \text { Saphire } \\
\text { 8\Debug } \backslash \text { source } \backslash \text { DelphiSource } \backslash \text { RaspCCF } \backslash\end{array}$ & 3 & \\
\hline CCFRCalc.pas & $\begin{array}{l}\text { C: } \backslash \text { Saphire } \\
8 \backslash \text { Debug } \backslash \text { source } \backslash \text { DelphiSource } \backslash \text { RaspCCF } \backslash\end{array}$ & 1 & \\
\hline CCFRCommon.pas & $\begin{array}{l}\text { C: } \backslash \text { Saphire } \\
\text { 8\Debug } \backslash \text { source } \backslash \text { DelphiSource } \backslash \text { RaspCCF } \backslash\end{array}$ & 2 & \\
\hline CCFRData.pas & $\begin{array}{l}\text { C: } \backslash \text { Saphire } \\
8 \backslash \text { Debug } \backslash \text { source } \backslash \text { DelphiSource } \backslash \text { RaspCCF } \backslash\end{array}$ & 4 & \\
\hline CCFRDebug.pas & $\begin{array}{l}\text { C: } \backslash \text { Saphire } \\
8 \backslash \text { Debug } \backslash \text { source } \backslash \text { DelphiSource } \backslash \text { RaspCCF } \backslash\end{array}$ & 3 & \\
\hline CCFRDLL.dpr & $\begin{array}{l}\text { C: } \backslash \text { Saphire } \\
8 \backslash \text { Debug } \backslash \text { source } \backslash \text { DelphiSource } \backslash \text { RaspCCF } \backslash\end{array}$ & 2 & \\
\hline CCFRInterface.pas & $\begin{array}{l}\text { C: } \backslash \text { Saphire } \\
8 \backslash \text { Debug } \backslash \text { source } \backslash \text { DelphiSource } \backslash \text { RaspCCF } \backslash\end{array}$ & 1 & \\
\hline CCFRLib.pas & $\begin{array}{l}\text { C: } \backslash \text { Saphire } \\
8 \backslash \text { Debug } \backslash \text { source } \backslash \text { DelphiSource } \backslash \text { RaspCCF } \backslash\end{array}$ & 2 & \\
\hline CCFRMGL.pas & $\begin{array}{l}\text { C: } \backslash \text { Saphire } \\
8 \backslash \text { Debug } \backslash \text { source } \backslash \text { DelphiSource } \backslash \text { RaspCCF } \backslash\end{array}$ & 4 & \\
\hline CCFRObj.pas & $\begin{array}{l}\mathrm{C}: \backslash \text { Saphire } \\
8 \backslash \text { Debug } \backslash \text { source } \backslash \text { DelphiSource } \backslash \text { EventEditing } \backslash\end{array}$ & 4 & \\
\hline CCFROutput.pas & $\begin{array}{l}\text { C: } \backslash \text { Saphire } \\
8 \backslash \text { Debug } \backslash \text { source } \backslash \text { DelphiSource } \backslash \text { RaspCCF } \backslash\end{array}$ & 3 & \\
\hline CCFRReport.pas & $\begin{array}{l}\text { C: } \backslash \text { Saphire } \\
8 \backslash \text { Debug } \backslash \text { source } \backslash \text { DelphiSource } \backslash \text { RaspCCF } \backslash\end{array}$ & 2 & \\
\hline CCFRStag.pas & $\begin{array}{l}\text { C: } \backslash \text { Saphire } \\
\text { 8\Debug } \backslash \text { source } \backslash \text { DelphiSource } \backslash \text { RaspCCF } \backslash\end{array}$ & 4 & \\
\hline CCFRUtil.pas & $\begin{array}{l}\text { C: } \backslash \text { Saphire } \\
\text { 8\Debug } \backslash \text { source } \backslash \text { DelphiSource } \backslash \text { RaspCCF } \backslash\end{array}$ & 5 & \\
\hline CCFRVBE.pas & $\begin{array}{l}\text { C: } \backslash \text { Saphire } \\
8 \backslash \text { Debug } \backslash \text { source } \backslash \text { DelphiSource } \backslash \text { RaspCCF } \backslash\end{array}$ & 2 & \\
\hline CFStag.pas & $\begin{array}{l}\mathrm{C}: \backslash \text { Saphire } \\
8 \backslash \text { Debug } \backslash \text { source } \backslash \text { DelphiSource } \backslash \text { PlugIns } \backslash\end{array}$ & 2 & \\
\hline CCLib.pas & C:\Saphire $8 \backslash$ Debug $\backslash$ source $\backslash$ & 2 & \\
\hline CCProcs.pas & $\mathrm{C}: \backslash$ Saphire $8 \backslash$ Debug $\backslash$ source $\backslash$ & 15 & \\
\hline CFGLib.pas & C: \Saphire 8\Debug $\backslash$ source $\backslash$ & 6 & \\
\hline CGMWin.pas & $\mathrm{C}: \backslash$ Saphire $8 \backslash$ Debug $\backslash$ source $\backslash$ & 10 & \\
\hline ChangeSet.pas & C:\Saphire $8 \backslash$ Debug $\backslash$ source $\backslash$ DelphiSource $\backslash$ & 3 & \\
\hline ChangeSet.pas & $\begin{array}{l}\text { C: } \backslash \text { Saphire } \\
8 \backslash \text { Debug } \backslash \text { source } \backslash \text { DelphiSource } \backslash \text { ProjObjects } \backslash\end{array}$ & 4 & \\
\hline
\end{tabular}




\begin{tabular}{|c|c|c|c|}
\hline \multirow[t]{2}{*}{ File Name } & \multirow[t]{2}{*}{ File Path } & \multicolumn{2}{|c|}{$\begin{array}{l}\text { Cyclomatic } \\
\text { Complexity }\end{array}$} \\
\hline & & v8.06.01 & v8.07.17 \\
\hline CheckLib.pas & C:|Saphire 8\Debug|source $\backslash$ & 1 & \\
\hline chgset.pas & C:|Saphire 8\Debug $\backslash$ source $\backslash$ DelphiSource $\backslash$ & 0 & \\
\hline ChgSetNodeEditor.pas & C:|Saphire 8\Debug \source $\mid$ DelphiSource $\backslash$ & 2 & \\
\hline ChgSetNodeEditor.pas & $\begin{array}{l}\text { C: } \backslash \text { Saphire } \\
8 \backslash \text { Debug } \backslash \text { source } \backslash \text { DelphiSource } \backslash \text { EventEditing } \backslash\end{array}$ & 3 & \\
\hline ClassCngTreeNode.pas & $\begin{array}{l}\text { C: } \backslash \text { Saphire } \\
8 \backslash \text { Debug } \backslash \text { source } \backslash \text { DelphiSource } \backslash \text { EventEditing } \backslash\end{array}$ & 6 & \\
\hline ClipBoard.pas & C:|Saphire 8\Debug|source $\backslash$ & 3 & \\
\hline CmndLine.pas & C: $\mid$ Saphire $8 \backslash$ Debug $\mid$ source $\backslash$ & 7 & \\
\hline CodeGenCommon.pas & C:|Saphire 8\Debug $\mid$ source $\backslash$ & 6 & \\
\hline CodeGenerator.pas & C: $\mid$ Saphire $8 \backslash$ Debug $\mid$ source $\backslash$ & 3 & \\
\hline CodeGenerator.pas & C: $\backslash$ Saphire $8 \backslash$ Debug $\backslash$ source $\backslash$ schemaObjects $\backslash$ & 3 & \\
\hline CodeLib.pas & C: $\mid$ Saphire $8 \backslash$ Debug $\mid$ source $\backslash$ & 13 & \\
\hline CodeMerg.pas & C:\Saphire 8\Debug $\backslash$ source $\backslash$ & 11 & \\
\hline Coder.pas & C: $\mid$ Saphire $8 \backslash$ Debug $\mid$ source $\backslash$ & 3 & \\
\hline CompareBE.pas & C: $\mid$ Saphire 8\Debug $\backslash$ source $\backslash$ DelphiSource $\backslash$ & 2 & \\
\hline CompareCS.pas & C: \Saphire 8\Debug \source\DelphiSource $\backslash$ & 2 & \\
\hline CompareDflt.pas & C: $\mid$ Saphire $8 \backslash$ Debug $\backslash$ source $\backslash$ DelphiSource $\backslash$ & 2 & \\
\hline CompareES.pas & C: \Saphire 8\Debug \source\DelphiSource $\backslash$ & 2 & \\
\hline CompareET.pas & C:ISaphire 8\Debug \source\DelphiSource $\backslash$ & 2 & \\
\hline CompareFT.pas & C: $\mid$ Saphire $8 \backslash$ Debug $\backslash$ source $\backslash$ DelphiSource $\backslash$ & 2 & \\
\hline CompareGT.pas & C:ISaphire 8\Debug \source $\backslash$ DelphiSource $\backslash$ & 2 & \\
\hline CompareHtml.pas & C:ISaphire 8\Debug \source\DelphiSource $\backslash$ & 9 & \\
\hline Compress.pas & C:|Saphire 8\Debug|source\} $&{6} &{ } \\
{\hline \text { Conversions.pas }} &{\text { C: } \mid \text { Saphire } 8 \backslash \text { Debug } \backslash \text { source } \backslash} &{6} &{ } \\
{\hline \text { Convert.pas }} &{\text { C:ISaphire 8\Debug|source\} } &{4} &{ } \\
{\hline \text { ConvertReal.pas }} &{\text { C: } \mid \text { Saphire } 8 \backslash \text { Debug } \backslash \text { source } \backslash} &{11} &{ } \\
{\hline \text { Crypto.pas }} &{\text { C: } \mid \text { Saphire } 8 \backslash \text { Debug } \backslash \text { source } \backslash} &{13} &{ } \\
{\hline \text { CtrlBttn.pas }} &{\text { C: } \backslash \text { Saphire } 8 \backslash \text { Debug } \backslash \text { source } \backslash} &{2} &{ } \\
{\hline \text { CtrlCBox.pas }} &{\text { C: } \mid \text { Saphire } 8 \backslash \text { Debug } \backslash \text { source } \backslash} &{2} &{ } \\
{\hline \text { CtrlComb.pas }} &{\text { C:\Saphire 8\Debug } \backslash \text { source } \backslash} &{2} &{ } \\
{\hline \text { CtrlEdit.pas }} &{\text { C: } \mid \text { Saphire } 8 \backslash \text { Debug } \backslash \text { source } \backslash} &{2} &{ } \\
{\hline \text { CtrlGrid.pas }} &{\text { C: } \backslash \text { Saphire } 8 \backslash \text { Debug } \backslash \text { source } \backslash} &{3} &{ } \\
{\hline \text { CtrlList.pas }} &{\text { C:\Saphire 8\Debug } \backslash \text { source } \backslash} &{3} &{ } \\
{\hline \text { CtrlLV.pas }} &{\text { C: } \mid \text { Saphire } 8 \backslash \text { Debug } \backslash \text { source } \backslash} &{2} &{ } \\
{\hline \text { CtrlRBtn.pas }} &{\text { C:|Saphire } 8 \backslash \text { Debug } \backslash \text { source } \backslash} &{2} &{ } \\
\hline\end{array}$
\end{tabular}

Page 10 


\begin{tabular}{|c|c|c|c|}
\hline \multirow[t]{2}{*}{ File Name } & \multirow[t]{2}{*}{ File Path } & \multicolumn{2}{|c|}{$\begin{array}{l}\text { Cyclomatic } \\
\text { Complexity }\end{array}$} \\
\hline & & v8.06.01 & v8.07.17 \\
\hline CtrlRich.pas & C:|Saphire $8 \backslash$ Debug $\mid$ source $\backslash$ & 3 & \\
\hline CtrlSBar.pas & C:|Saphire $8 \backslash$ Debug $\mid$ source $\backslash$ & 2 & \\
\hline CtrlSppt.pas & C:|Saphire 8\Debug|source\} $&{3} &{ } \\
{\hline \text { CtrlStat.pas }} &{\text { C: } \mid \text { Saphire } 8 \backslash \text { Debug } \mid \text { source } \backslash} &{1} &{ } \\
{\hline \text { CtrlTree.pas }} &{\text { C:|Saphire 8\Debug|source } \backslash} &{3} &{ } \\
{\hline \text { CtrlWin.pas }} &{\text { C:|Saphire 8\Debug|source\} } &{2} &{ } \\
{\hline \text { CustCntl.pas }} &{\text { C:|Saphire 8\Debug|source\} } &{0} &{ } \\
{\hline \text { CustRptLib.pas }} &{\text { C:ISaphire 8\Debug \source \DelphiSource } \backslash} &{37} &{ } \\
{\hline \text { DataPack.pas }} &{\text { C:\Saphire 8\Debug \source \hfdllı }} &{0} &{ } \\
{\hline \text { DBLoad.pas }} &{\text { C:|Saphire 8\Debug|source } \backslash} &{9} &{ } \\
{\hline \text { DBMerge.pas }} &{\text { C:|Saphire 8\Debug|source\} } &{6} &{ } \\
{\hline \text { DCompile.pas }} &{\text { C:|Saphire } 8 \backslash \text { Debug } \mid \text { source } \backslash} &{10} &{ } \\
{\hline \text { DDAccess.pas }} &{\text { C:ISaphire 8\Debug|source\} } &{9} &{ } \\
{\hline \text { DDUniversal.pas }} &{\text { C:|Saphire 8\Debug|source\} } &{4} &{ } \\
{\hline \text { DesignOperation.pas }} &{\begin{array}{l}\text { C: } \mid \text { Saphire } \\
8 \backslash \text { Debug } \backslash \text { source } \backslash \text { DelphiSource } \backslash \text { GraphicalEditi } \\
\text { ng } \backslash\end{array}} &{1} &{ } \\
{\hline \text { Dialog.pas }} &{\text { C:|Saphire 8\Debug|source\} } &{2} &{ } \\
{\hline \text { Disklib.pas }} &{\text { C:ISaphire 8\Debug } \mid \text { source } \backslash} &{4} &{ } \\
{\hline \text { Display.pas }} &{\text { C:ISaphire 8\Debug|source\} } &{5} &{ } \\
{\hline \text { DisplayW.pas }} &{\text { C: } \mid \text { Saphire } 8 \backslash \text { Debug } \backslash \text { source } \backslash} &{7} &{ } \\
{\hline \text { dlgConfirmReplace.pas }} &{\begin{array}{l}\text { C: } \backslash \text { Saphire } \\
\text { 8\Debug } \backslash \text { source } \backslash \text { DelphiSource } \backslash \text { RulesEditor } \backslash\end{array}} &{2} &{ } \\
{\hline \text { dlgReplaceText.pas }} &{\begin{array}{l}\text { C:\Saphire } \\
\text { 8\Debug } \backslash \text { source } \backslash \text { DelphiSource } \backslash \text { RulesEditor } \backslash\end{array}} &{3} &{ } \\
{\hline \text { DlgSageSTMain.pas }} &{\text { C:ISaphire 8\Debug|source\} } &{0} &{ } \\
{\hline \text { dlgSearchText.pas }} &{\begin{array}{l}\text { C: } \mid \text { Saphire } \\
\text { 8\Debug } \backslash \text { source } \backslash \text { DelphiSource } \backslash \text { RulesEditor } \backslash\end{array}} &{2} &{ } \\
{\hline \text { DMCat.pas }} &{\text { C: } \mid \text { Saphire } 8 \backslash \text { Debug } \backslash \text { source } \backslash \text { DelphiSource } \backslash} &{2} &{ } \\
{\hline \text { DMRegExpMatching.pas }} &{\text { C:ISaphire 8\Debug \source\DelphiSource } \backslash} &{1} &{ } \\
{\hline \text { DosLib.pas }} &{\text { C:|Saphire 8\Debug } \mid \text { source } \backslash} &{4} &{ } \\
{\hline \text { DTstGen.pas }} &{\begin{array}{l}\text { C: } \backslash \text { Saphire } \\
\text { 8\Debug } \backslash \text { source } \backslash \text { DelphiSource } \backslash \text { BddItems } \backslash \text { Has } \\
\text { hTableLib } \backslash\end{array}} &{3} &{ } \\
{\hline \text { DWLib.pas }} &{\text { C: } \mid \text { Saphire } 8 \backslash \text { Debug } \mid \text { source } \backslash} &{4} &{ } \\
{\hline \text { DWSpec.pas }} &{\text { C:|Saphire 8\Debug|source\} } &{0} &{ } \\
{\hline \text { EditAnalysisPoint.pas }} &{\begin{array}{l}\text { C: } \mid \text { Saphire } \\
8 \backslash \text { Debug } \backslash \text { source } \backslash \text { DelphiSource } \backslash \text { GraphicalEditi } \\
\text { ng } \backslash\end{array}} &{3} &{ } \\
\hline\end{array}$
\end{tabular}




\begin{tabular}{|c|c|c|c|}
\hline \multirow[t]{2}{*}{ File Name } & \multirow[t]{2}{*}{ File Path } & \multicolumn{2}{|c|}{$\begin{array}{l}\text { Cyclomatic } \\
\text { Complexity }\end{array}$} \\
\hline & & v8.06.01 & v8.07.17 \\
\hline EditETAnalysisPoint.pas & $\begin{array}{l}\text { C: } \backslash \text { Saphire } \\
8 \backslash \text { Debug } \backslash \text { source } \backslash \text { DelphiSource } \backslash \text { GraphicalEditi } \\
n g \backslash\end{array}$ & 3 & \\
\hline EditEvent.pas & $\begin{array}{l}\text { C: } \backslash \text { Saphire } \\
8 \backslash \text { Debug } \backslash \text { source } \backslash \text { DelphiSource } \backslash \text { GraphicalEditi } \\
\text { ng } \backslash\end{array}$ & 3 & \\
\hline EditEventEndstate.pas & $\begin{array}{l}\text { C: } \backslash \text { Saphire } \\
8 \backslash \text { Debug } \backslash \text { source } \backslash \text { DelphiSource } \backslash \text { GraphicalEditi } \\
\text { ng } \backslash\end{array}$ & 3 & \\
\hline EditEventEndstateCol.pas & $\begin{array}{l}\text { C: } \backslash \text { Saphire } \\
8 \backslash \text { Debug } \backslash \text { source } \backslash \text { DelphiSource } \backslash \text { GraphicalEditi } \\
\text { ng }\end{array}$ & 2 & \\
\hline EditEventTree.pas & $\begin{array}{l}\text { C: } \backslash \text { Saphire } \\
8 \backslash \text { Debug } \backslash \text { source } \backslash \text { DelphiSource } \backslash \text { GraphicalEditi } \\
\text { ng }\end{array}$ & 3 & \\
\hline EditFaultTreeObject.pas & $\begin{array}{l}\text { C: } \backslash \text { Saphire } \\
8 \backslash \text { Debug } \backslash \text { source } \backslash \text { DelphiSource } \backslash \text { GraphicalEditi } \\
\text { ng }\end{array}$ & 6 & \\
\hline EditLib.pas & $\mathrm{C}: \backslash$ Saphire $8 \backslash$ Debug $\backslash$ source $\backslash$ & 6 & \\
\hline EditRBlockObject.pas & C: $\backslash$ Saphire $8 \backslash$ Debug $\backslash$ source $\backslash$ DelphiSource $\backslash$ & 2 & \\
\hline EnvironmentX.pas & $\mathrm{C}: \backslash$ Saphire $8 \backslash$ Debug $\backslash$ source $\backslash$ & 4 & \\
\hline ErrorLog.pas & C:\Saphire $8 \backslash$ Debug $\backslash$ source $\backslash$ & 7 & \\
\hline ESCatEdit.pas & C:\Saphire $8 \backslash$ Debug $\backslash$ source $\backslash$ DelphiSource $\backslash$ & 5 & \\
\hline ETGroupObj.pas & $\begin{array}{l}\text { C: } \backslash \text { Saphire } \\
8 \backslash \text { Debug } \backslash \text { source } \backslash \text { DelphiSource } \backslash \text { ProjObjects } \backslash\end{array}$ & 3 & \\
\hline ETObject.pas & $\begin{array}{l}\text { C: } \backslash \text { Saphire } \\
8 \backslash \text { Debug } \backslash \text { source } \backslash \text { DelphiSource } \backslash \text { ProjObjects } \backslash\end{array}$ & 3 & \\
\hline EventEndObject.pas & $\begin{array}{l}\text { C: } \backslash \text { Saphire } \\
8 \backslash \text { Debug } \backslash \text { source } \backslash \text { DelphiSource } \backslash \text { GraphicalEditi } \\
\text { ng } \backslash\end{array}$ & 3 & \\
\hline EventIns.pas & $\mathrm{C}: \backslash$ Saphire $8 \backslash$ Debug $\backslash$ source $\backslash$ & 6 & \\
\hline EventObject.pas & $\begin{array}{l}\text { C: } \backslash \text { Saphire } \\
\text { 8\Debug } \backslash \text { source } \backslash \text { DelphiSource } \backslash \text { GraphicalEditi } \\
\text { ng }\end{array}$ & 2 & \\
\hline EventScheduler.pas & C:\Saphire $8 \backslash$ Debug $\backslash$ source $\backslash$ DelphiSource $\backslash$ & 9 & \\
\hline EventSetObj.pas & C:\Saphire $8 \backslash$ Debug $\backslash$ source $\backslash$ DelphiSource $\backslash$ & 3 & \\
\hline EventTreeObject.pas & $\begin{array}{l}\text { C: } \backslash \text { Saphire } \\
8 \backslash \text { Debug } \backslash \text { source } \backslash \text { DelphiSource } \backslash \text { GraphicalEditi } \\
\text { ng }\end{array}$ & 5 & \\
\hline EventView1.pas & $\mathrm{C}: \backslash$ Saphire $8 \backslash$ Debug $\backslash$ source $\backslash$ DelphiSource $\backslash$ & 0 & \\
\hline EvProbList.pas & $\mathrm{C}: \backslash$ Saphire $8 \backslash$ Debug $\backslash$ source $\backslash$ DelphiSource $\backslash$ & 3 & \\
\hline EvTCatObj.pas & C:\Saphire $8 \backslash$ Debug $\backslash$ source $\backslash$ DelphiSource $\backslash$ & 2 & \\
\hline ExcelOut.pas & C:\Saphire 8\Debug $\backslash$ source $\backslash$ DelphiSource $\backslash$ & 2 & \\
\hline
\end{tabular}




\begin{tabular}{|c|c|c|c|}
\hline \multirow[t]{2}{*}{ File Name } & \multirow[t]{2}{*}{ File Path } & \multicolumn{2}{|c|}{$\begin{array}{l}\text { Cyclomatic } \\
\text { Complexity }\end{array}$} \\
\hline & & v8.06.01 & v8.07.17 \\
\hline Exec.pas & $\mathrm{C}: \backslash$ Saphire $8 \backslash$ Debug $\backslash$ source $\backslash$ & 1 & \\
\hline ExpOpt.pas & $\mathrm{C}: \backslash$ Saphire $8 \backslash$ Debug $\backslash$ source $\backslash$ & 8 & \\
\hline ExStorageX.pas & $\mathrm{C}: \backslash$ Saphire $8 \backslash$ Debug $\backslash$ source $\backslash$ & 4 & \\
\hline EZDSLCTS.PAS & $\begin{array}{l}\text { C: } \backslash \text { Saphire } \\
8 \backslash \text { Debug } \backslash \text { source } \backslash \text { DelphiSource } \backslash B d d I t e m s \backslash \text { Has } \\
\text { hTableLib } \backslash\end{array}$ & 0 & \\
\hline FastMM4.pas & C: $\backslash$ Saphire $8 \backslash$ Debug $\backslash$ source $\backslash$ fastmm $\backslash$ & 6 & \\
\hline FastMM4Messages.pas & C:\Saphire $8 \backslash$ Debug $\backslash$ source $\backslash$ fastmm $\backslash$ & 0 & \\
\hline FaultTreeObject.pas & $\begin{array}{l}\text { C: } \backslash \text { Saphire } \\
8 \backslash \text { Debug } \backslash \text { source } \backslash \text { DelphiSource } \backslash \text { GraphicalEditi } \\
\text { ng } \backslash\end{array}$ & 5 & \\
\hline FileEdit.pas & $\mathrm{C}: \backslash$ Saphire $8 \backslash$ Debug $\backslash$ source $\backslash$ & 3 & \\
\hline FileFunc.pas & C: $\backslash$ Saphire $8 \backslash$ Debug $\backslash$ source $\backslash$ & 6 & \\
\hline FileQuery.pas & $\mathrm{C}: \backslash$ Saphire $8 \backslash$ Debug $\backslash$ source $\backslash$ & 2 & \\
\hline Files.pas & C: $\mid$ Saphire $8 \backslash$ Debug $\backslash$ source $\backslash$ & 4 & \\
\hline FilesOp.pas & C:\Saphire $8 \backslash$ Debug $\backslash$ source $\backslash$ DelphiSource $\backslash$ & 4 & \\
\hline FileUtil.pas & $\mathrm{C}: \backslash$ Saphire $8 \backslash$ Debug $\backslash$ source $\backslash$ & 7 & \\
\hline FilterFunctions.pas & C:\Saphire $8 \backslash$ Debug $\backslash$ source $\backslash$ DelphiSource $\backslash$ & 3 & \\
\hline FixLib.pas & C:\Saphire $8 \backslash$ Debug $\backslash$ source $\backslash$ & 9 & \\
\hline FlagLib.pas & C: \Saphire $8 \backslash$ Debug $\backslash$ source $\backslash$ & 4 & \\
\hline FldFlags.pas & C:\Saphire $8 \backslash$ Debug $\backslash$ source $\backslash$ & 8 & \\
\hline Fonts.pas & C:\Saphire $8 \backslash$ Debug $\backslash$ source $\backslash$ & 4 & \\
\hline Form_BDD_FT_Solve.pas & $\begin{array}{l}\text { C: } \backslash \text { Saphire } \\
8 \backslash \text { Debug } \backslash \text { source } \backslash \text { DelphiSource } \backslash B d d I t e m s \backslash\end{array}$ & 3 & \\
\hline Form_ErrorLog.pas & C:\Saphire $8 \backslash$ Debug $\backslash$ source $\backslash$ DelphiSource $\backslash$ & 2 & \\
\hline Form_Histograms.pas & C: $\backslash$ Saphire $8 \backslash$ Debug $\backslash$ source $\backslash$ DelphiSource $\backslash$ & 3 & \\
\hline Form_SeqProperties.pas & C:\Saphire $8 \backslash$ Debug $\backslash$ source $\backslash$ DelphiSource $\backslash$ & 2 & \\
\hline FormAccidentMatrix.pas & C:\Saphire $8 \backslash$ Debug $\backslash$ source $\backslash$ DelphiSource $\backslash$ & 5 & \\
\hline FormAnalysisType.pas & C:\Saphire $8 \backslash$ Debug $\backslash$ source $\backslash$ DelphiSource $\backslash$ & 5 & \\
\hline FormAnalysisTypeEdit.pas & C:\Saphire $8 \backslash$ Debug $\backslash$ source $\backslash$ DelphiSource $\backslash$ & 1 & \\
\hline FormASP.pas & C:\Saphire $8 \backslash$ Debug $\backslash$ source $\backslash$ DelphiSource $\backslash$ & 7 & \\
\hline FormASP1.pas & $\mathrm{C}: \backslash$ Saphire $8 \backslash$ Debug $\backslash$ source $\backslash$ DelphiSource $\backslash$ & 4 & \\
\hline FormASP2.pas & C: $\backslash$ Saphire $8 \backslash$ Debug $\backslash$ source $\backslash$ DelphiSource $\backslash$ & 2 & \\
\hline FormASP3.pas & C:\Saphire $8 \backslash$ Debug $\backslash$ source $\backslash$ DelphiSource $\backslash$ & 4 & \\
\hline FormBE.pas & C:\Saphire $8 \backslash$ Debug $\backslash$ source $\backslash$ DelphiSource $\backslash$ & 2 & \\
\hline FormBEData.pas & C: \Saphire 8\Debug \source \hfdll \} $&{5} &{ } \\
{\hline \text { FormBEDataEdit.pas }} &{\begin{array}{l}\mathrm{C}: \backslash \text { Saphire } \\
8 \backslash \text { Debug } \backslash \text { source } \backslash \text { DelphiSource } \backslash \text { EventEditing } \backslash\end{array}} &{5} &{ } \\
$\hline
\end{tabular}




\begin{tabular}{|c|c|c|c|}
\hline \multirow[t]{2}{*}{ File Name } & \multirow[t]{2}{*}{ File Path } & \multicolumn{2}{|c|}{$\begin{array}{l}\text { Cyclomatic } \\
\text { Complexity }\end{array}$} \\
\hline & & v8.06.01 & v8.07.17 \\
\hline FormBookmarkAdd.pas & C:\Saphire $8 \backslash$ Debug $\backslash$ source $\backslash$ DelphiSource $\backslash$ & 1 & \\
\hline FormCategories.pas & C:\Saphire $8 \backslash$ Debug $\backslash$ source $\backslash$ DelphiSource $\backslash$ & 3 & \\
\hline FormCategoryEdit.pas & C:\Saphire $8 \backslash$ Debug $\backslash$ source $\backslash$ DelphiSource $\backslash$ & 3 & \\
\hline FormChangeBE.pas & $\mathrm{C}: \backslash$ Saphire $8 \backslash$ Debug $\backslash$ source $\backslash$ DelphiSource $\backslash$ & 2 & \\
\hline FormChangeSet.pas & $\begin{array}{l}C: \backslash \text { Saphire } \\
8 \backslash \text { Debug } \backslash \text { source } \backslash \text { DelphiSource } \backslash \text { EventEditing } \backslash\end{array}$ & 3 & \\
\hline FormChangeSetEdit.pas & $\begin{array}{l}\text { C: } \backslash \text { Saphire } \\
8 \backslash \text { Debug } \backslash \text { source } \backslash \text { DelphiSource } \backslash \text { EventEditing } \backslash\end{array}$ & 2 & \\
\hline FormChk.pas & C:\Saphire $8 \backslash$ Debug $\backslash$ source $\backslash$ & 1 & \\
\hline FormCompareDBs.pas & C:\Saphire $8 \backslash$ Debug $\backslash$ source $\backslash$ DelphiSource $\backslash$ & 3 & \\
\hline FormCustomPrint.pas & $\begin{array}{l}\text { C: } \backslash \text { Saphire } \\
8 \backslash \text { Debug } \backslash \text { source } \backslash \text { DelphiSource } \backslash \text { GraphicalEditi } \\
\text { ng } \backslash\end{array}$ & 5 & \\
\hline FormCustReportList.pas & C:\Saphire $8 \backslash$ Debug $\backslash$ source $\backslash$ DelphiSource $\backslash$ & 3 & \\
\hline FormCustRptEdit.pas & C:\Saphire $8 \backslash$ Debug $\backslash$ source $\backslash$ DelphiSource $\backslash$ & 3 & \\
\hline FormCutSetViewer.pas & C:\Saphire $8 \backslash$ Debug $\backslash$ source $\backslash$ DelphiSource $\backslash$ & 5 & \\
\hline FormCutsetViewerES.pas & C:\Saphire $8 \backslash$ Debug $\backslash$ source $\backslash$ DelphiSource $\backslash$ & 4 & \\
\hline FormCutSetViewerET.pas & C:\Saphire $8 \backslash$ Debug $\backslash$ source $\backslash$ DelphiSource $\backslash$ & 4 & \\
\hline FormCutSetViewerFT.pas & C:\Saphire $8 \backslash$ Debug $\backslash$ source $\backslash$ DelphiSource $\backslash$ & 3 & \\
\hline FormDesignEdtBase.pas & $\begin{array}{l}\text { C: } \backslash \text { Saphire } \\
8 \backslash \text { Debug } \backslash \text { source } \backslash \text { DelphiSource } \backslash \text { GraphicalEditi } \\
n g \backslash\end{array}$ & 2 & \\
\hline FormDocManager.pas & C:\Saphire $8 \backslash$ Debug $\backslash$ source $\backslash$ DelphiSource $\backslash$ & 6 & \\
\hline FormEditBookmarks.pas & C:\Saphire $8 \backslash$ Debug $\backslash$ source $\backslash$ DelphiSource $\backslash$ & 3 & \\
\hline FormEditETGroups.pas & C:\Saphire $8 \backslash$ Debug $\backslash$ source $\backslash$ DelphiSource $\backslash$ & 3 & \\
\hline FormEditEventGroups.pas & C:\Saphire $8 \backslash$ Debug $\backslash$ source $\backslash$ DelphiSource $\backslash$ & 2 & \\
\hline FormEndStateEdit.pas & C:\Saphire $8 \backslash$ Debug $\backslash$ source $\backslash$ DelphiSource $\backslash$ & 4 & \\
\hline FormESCategory.pas & $\mathrm{C}: \backslash$ Saphire $8 \backslash$ Debug $\backslash$ source $\backslash$ DelphiSource $\backslash$ & 2 & \\
\hline FormET.pas & C:\Saphire $8 \backslash$ Debug $\backslash$ source $\backslash$ DelphiSource $\backslash$ & 2 & \\
\hline FormETEditor.pas & $\begin{array}{l}\text { C: } \backslash \text { Saphire } \\
8 \backslash \text { Debug } \backslash \text { source } \backslash \text { DelphiSource } \backslash \text { GraphicalEditi } \\
\text { ng } \backslash\end{array}$ & 4 & \\
\hline FormETGraphicalDisp.pas & $\begin{array}{l}\text { C: } \backslash \text { Saphire } \\
8 \backslash \text { Debug } \backslash \text { source } \backslash \text { DelphiSource } \backslash \text { GraphicalEditi } \\
\text { ng } \backslash\end{array}$ & 6 & \\
\hline FormETGraphicOnly.pas & $\begin{array}{l}\text { C: } \backslash \text { Saphire } \\
8 \backslash \text { Debug } \backslash \text { source } \backslash \text { DelphiSource } \backslash \text { GraphicalEditi } \\
\text { ng } \backslash\end{array}$ & 2 & \\
\hline FormExtractProject.pas & C:\Saphire $8 \backslash$ Debug $\backslash$ source $\backslash$ DelphiSource $\backslash$ & 4 & \\
\hline FormFlagSetEdit.pas & C:ISaphire & 2 & \\
\hline
\end{tabular}




\begin{tabular}{|c|c|c|c|}
\hline \multirow[t]{2}{*}{ File Name } & \multirow[t]{2}{*}{ File Path } & \multicolumn{2}{|c|}{$\begin{array}{l}\text { Cyclomatic } \\
\text { Complexity }\end{array}$} \\
\hline & & v8.06.01 & v8.07.17 \\
\hline & $8 \backslash$ Debug $\backslash$ source $\backslash$ DelphiSource $\backslash$ EventEditing $\backslash$ & & \\
\hline FormFlagSets.pas & C:\Saphire $8 \backslash$ Debug $\backslash$ source $\backslash$ DelphiSource $\backslash$ & 3 & \\
\hline FormFT.pas & C:\Saphire $8 \backslash$ Debug $\backslash$ source $\backslash$ DelphiSource $\backslash$ & 4 & \\
\hline FormFTCSGraphicalDisp.pas & $\begin{array}{l}\text { C: } \backslash \text { Saphire } \\
8 \backslash \text { Debug } \backslash \text { source } \backslash \text { DelphiSource } \backslash \text { GraphicalEditi } \\
\text { ng } \backslash\end{array}$ & 3 & \\
\hline FormFTCSPathViewer.pas & $\begin{array}{l}\text { C: } \backslash \text { Saphire } \\
8 \backslash \text { Debug } \backslash \text { source } \backslash \text { DelphiSource } \backslash \text { GraphicalEditi } \\
\text { ng } \backslash\end{array}$ & 1 & \\
\hline FormFTEditor.pas & $\begin{array}{l}\text { C: } \backslash \text { Saphire } \\
8 \backslash \text { Debug } \backslash \text { source } \backslash \text { DelphiSource } \backslash \text { GraphicalEditi } \\
\text { ng } \backslash\end{array}$ & 3 & \\
\hline FormFTGraphicalDisp.pas & $\begin{array}{l}\text { C: } \backslash \text { Saphire } \\
8 \backslash \text { Debug } \backslash \text { source } \backslash \text { DelphiSource } \backslash \text { GraphicalEditi } \\
n g \backslash\end{array}$ & 5 & \\
\hline FormFTGraphicOnly.pas & $\begin{array}{l}\text { C: } \backslash \text { Saphire } \\
8 \backslash \text { Debug } \backslash \text { source } \backslash \text { DelphiSource } \backslash \text { GraphicalEditi } \\
\text { ng } \backslash\end{array}$ & 2 & \\
\hline FormGenAn.pas & C:\Saphire 8\Debug $\backslash$ source $\backslash$ DelphiSource $\backslash$ & 3 & \\
\hline FormGenAn1.pas & C:\Saphire $8 \backslash$ Debug $\backslash$ source $\backslash$ DelphiSource $\backslash$ & 3 & \\
\hline FormGenAn2.pas & C:\Saphire $8 \backslash$ Debug $\backslash$ source $\backslash$ DelphiSource $\backslash$ & 2 & \\
\hline FormGenAn3.pas & C:\Saphire 8\Debug $\backslash$ source $\backslash$ DelphiSource $\backslash$ & 9 & \\
\hline FormGenAn4.pas & C:\Saphire $8 \backslash$ Debug $\backslash$ source $\backslash$ DelphiSource $\backslash$ & 4 & \\
\hline FormGraphicalDispBase.pas & $\begin{array}{l}\text { C: } \backslash \text { Saphire } \\
8 \backslash \text { Debug } \backslash \text { source } \backslash \text { DelphiSource } \backslash \text { GraphicalEditi } \\
\text { ng } \backslash\end{array}$ & 4 & \\
\hline FormHistogramEdit.pas & $\mathrm{C}: \backslash$ Saphire $8 \backslash$ Debug $\backslash$ source $\backslash$ DelphiSource $\backslash$ & 4 & \\
\hline FormHistograms.pas & C:\Saphire $8 \backslash$ Debug $\backslash$ source $\backslash$ DelphiSource $\backslash$ & 3 & \\
\hline FormImpExp.pas & C:\Saphire $8 \backslash$ Debug $\backslash$ source $\backslash$ DelphiSource $\backslash$ & 2 & \\
\hline FormImport.pas & C:\Saphire $8 \backslash$ Debug $\backslash$ source $\backslash$ DelphiSource $\backslash$ & 1 & \\
\hline FormImportanceM.pas & C:\Saphire $8 \backslash$ Debug $\backslash$ source $\backslash$ DelphiSource $\backslash$ & 5 & \\
\hline FormLib.pas & C: $\backslash$ Saphire $8 \backslash$ Debug $\backslash$ source $\backslash$ & 7 & \\
\hline FormLink.pas & C:\Saphire $8 \backslash$ Debug $\backslash$ source $\backslash$ DelphiSource $\backslash$ & 4 & \\
\hline FormLinkProgress.pas & C:\Saphire $8 \backslash$ Debug $\backslash$ source $\backslash$ DelphiSource $\backslash$ & 2 & \\
\hline FormLinkResults.pas & C:\Saphire $8 \backslash$ Debug $\backslash$ source $\backslash$ DelphiSource $\backslash$ & 3 & \\
\hline FormLoadExtProb.pas & $\begin{array}{l}\text { C: } \backslash \text { Saphire } \\
8 \backslash \text { Debug } \backslash \text { source } \backslash \text { DelphiSource } \backslash I m p o r t E x p o r t \backslash\end{array}$ & 2 & \\
\hline FormLoadExtract.pas & C:\Saphire $8 \backslash$ Debug $\backslash$ source $\backslash$ DelphiSource $\backslash$ & 15 & \\
\hline FormLoadExtractProbs.pas & C:\Saphire $8 \backslash$ Debug $\backslash$ source $\backslash$ DelphiSource $\backslash$ & 1 & \\
\hline FormMacroManager.pas & C:\Saphire $8 \backslash$ Debug $\backslash$ source $\backslash$ DelphiSource $\backslash$ & 5 & \\
\hline
\end{tabular}




\begin{tabular}{|c|c|c|c|}
\hline \multirow[t]{2}{*}{ File Name } & \multirow[t]{2}{*}{ File Path } & \multicolumn{2}{|c|}{$\begin{array}{l}\text { Cyclomatic } \\
\text { Complexity }\end{array}$} \\
\hline & & v8.06.01 & v8.07.17 \\
\hline FormMacroRunner.pas & C:|Saphire $8 \backslash$ Debug $\backslash$ source $\backslash$ DelphiSource $\backslash$ & 19 & \\
\hline FormMain.pas & C: $\mid$ Saphire $8 \backslash$ Debug $\backslash$ source $\backslash$ DelphiSource $\backslash$ & 4 & \\
\hline FormMinMax.pas & C:|Saphire 8\Debug $\backslash$ source $\backslash$ DelphiSource $\backslash$ & 3 & \\
\hline FormModelDataEdit.pas & $\begin{array}{l}\text { C: } \backslash \text { Saphire } \\
\text { 8\Debug } \backslash \text { source } \backslash \text { DelphiSource } \backslash \text { EventEditing } \backslash\end{array}$ & 3 & \\
\hline FormModelDataTab.pas & $\begin{array}{l}\text { C: } \backslash \text { Saphire } \\
8 \backslash \text { Debug } \backslash \text { source } \backslash \text { DelphiSource } \backslash \text { EventEditing } \backslash\end{array}$ & 3 & \\
\hline FormModelType.pas & C: $\mid$ Saphire $8 \backslash$ Debug $\backslash$ source $\backslash$ DelphiSource $\backslash$ & 4 & \\
\hline FormModelTypeEdit.pas & C: $\mid$ Saphire $8 \backslash$ Debug $\backslash$ source $\backslash$ DelphiSource $\backslash$ & 2 & \\
\hline FormNameDesc.pas & C:|Saphire 8\Debug $\mid$ source $\backslash$ DelphiSource $\backslash$ & 0 & \\
\hline FormNewItemData.pas & $\begin{array}{l}\text { C: } \backslash \text { Saphire } \\
8 \backslash \text { Debug } \backslash \text { source } \backslash \text { DelphiSource } \backslash \text { GraphicalEditi } \\
\text { ng } \backslash\end{array}$ & 1 & \\
\hline FormNewProject.pas & C: $\backslash$ Saphire $8 \backslash$ Debug $\backslash$ source $\backslash$ DelphiSource $\backslash$ & 3 & \\
\hline FormPhase.pas & C: $\mid$ Saphire $8 \backslash$ Debug $\backslash$ source $\backslash$ DelphiSource $\backslash$ & 4 & \\
\hline FormPhaseEdit.pas & C: \Saphire 8\Debug \source \DelphiSource $\backslash$ & 2 & \\
\hline FormPID.pas & C: $\mid$ Saphire $8 \backslash$ Debug $\backslash$ source $\backslash$ DelphiSource $\backslash$ & 1 & \\
\hline FormProjCheck.pas & C:ISaphire 8\Debug \source \DelphiSource $\backslash$ & 7 & \\
\hline FormProjectUpdate.pas & C: $\mid$ Saphire $8 \backslash$ Debug $\mid$ source $\backslash$ DelphiSource $\backslash$ & 4 & \\
\hline FormPublish.pas & C: \Saphire 8\Debug \source \DelphiSource $\backslash$ & 5 & \\
\hline FormPublish2.pas & C:|Saphire 8\Debug $\mid$ source $\backslash$ DelphiSource $\backslash$ & 7 & \\
\hline FormQuickEditEventES.pas & $\begin{array}{l}\text { C: } \backslash \text { Saphire } \\
8 \backslash \text { Debug } \backslash \text { source } \backslash \text { DelphiSource } \backslash \text { GraphicalEditi } \\
\text { ng } \backslash\end{array}$ & 2 & \\
\hline FormQuickEditEventESCol.pas & $\begin{array}{l}\text { C: } \backslash \text { Saphire } \\
\text { 8\Debug } \backslash \text { source } \backslash \text { DelphiSource } \backslash \text { GraphicalEditi } \\
\text { ng } \backslash\end{array}$ & 2 & \\
\hline FormQuickEditEventTree.pas & $\begin{array}{l}\text { C: } \backslash \text { Saphire } \\
8 \backslash \text { Debug } \backslash \text { source } \backslash \text { DelphiSource } \backslash \text { GraphicalEditi } \\
\text { ng } \backslash\end{array}$ & 2 & \\
\hline FormQuickEditFaultTree.pas & $\begin{array}{l}\text { C: } \mid \text { Saphire } \\
8 \backslash \text { Debug } \backslash \text { source } \backslash \text { DelphiSource } \backslash \text { GraphicalEditi } \\
\text { ng } \backslash\end{array}$ & 2 & \\
\hline FormQuickEditTopEvent.pas & $\begin{array}{l}\text { C: } \backslash \text { Saphire } \\
8 \backslash \text { Debug } \backslash \text { source } \backslash \text { DelphiSource } \backslash \text { GraphicalEditi } \\
\text { ng } \backslash\end{array}$ & 2 & \\
\hline FormQuickSearch.pas & $\begin{array}{l}\text { C: } \backslash \text { Saphire } \\
8 \backslash \text { Debug } \backslash \text { source } \backslash \text { DelphiSource } \backslash \text { GraphicalEditi } \\
\text { ng } \backslash\end{array}$ & 1 & \\
\hline FormRaspCmpd.pas & C: $\mid$ Saphire 8\Debug $\backslash$ source $\backslash$ DelphiSource $\backslash$ & 3 & \\
\hline FormRebuildUpgrade.pas & C: $\mid$ Saphire $8 \backslash$ Debug $\backslash$ source $\backslash$ DelphiSource $\backslash$ & 2 & \\
\hline
\end{tabular}




\begin{tabular}{|c|c|c|c|}
\hline \multirow[t]{2}{*}{ File Name } & \multirow[t]{2}{*}{ File Path } & \multicolumn{2}{|c|}{$\begin{array}{l}\text { Cyclomatic } \\
\text { Complexity }\end{array}$} \\
\hline & & v8.06.01 & v8.07.17 \\
\hline FormResultsViewer.pas & $\mathrm{C}: \backslash$ Saphire $8 \backslash$ Debug $\backslash$ source $\backslash$ DelphiSource $\backslash$ & 2 & \\
\hline FormResultsViewer.pas & $\begin{array}{l}\text { C: } \backslash \text { Saphire } \\
8 \backslash \text { Debug } \backslash \text { source } \backslash \text { DelphiSource } \backslash \text { ResultsViewer } \\
\backslash\end{array}$ & 3 & \\
\hline FormResultsViewerES.pas & $\begin{array}{l}\text { C: } \backslash \text { Saphire } \\
8 \backslash \text { Debug } \backslash \text { source } \backslash \text { DelphiSource } \backslash \text { ResultsViewer } \\
\backslash\end{array}$ & 3 & \\
\hline FormResultsViewerET.pas & $\begin{array}{l}\text { C: } \backslash \text { Saphire } \\
8 \backslash \text { Debug } \backslash \text { source } \backslash \text { DelphiSource } \backslash \text { ResultsViewer } \\
\backslash\end{array}$ & 4 & \\
\hline FormResultsViewerFT.pas & $\begin{array}{l}\text { C: } \backslash \text { Saphire } \\
8 \backslash \text { Debug } \backslash \text { source } \backslash \text { DelphiSource } \backslash \text { ResultsViewer } \\
\backslash\end{array}$ & 2 & \\
\hline FormRuleEdETLink.pas & $\begin{array}{l}C: \backslash \text { Saphire } \\
8 \backslash \text { Debug } \backslash \text { source } \backslash \text { DelphiSource } \backslash \text { RulesEditor } \backslash\end{array}$ & 2 & \\
\hline FormRuleEdETPart.pas & $\begin{array}{l}\text { C:\Saphire } \\
8 \backslash \text { Debug } \backslash \text { source } \backslash \text { DelphiSource } \backslash \text { RulesEditor } \backslash\end{array}$ & 1 & \\
\hline FormRuleEdETRec.pas & $\begin{array}{l}\text { C:\Saphire } \\
8 \backslash \text { Debug } \backslash \text { source } \backslash \text { DelphiSource } \backslash \text { RulesEditor } \backslash\end{array}$ & 1 & \\
\hline FormRuleEdFTRec.pas & $\begin{array}{l}\text { C:\Saphire } \\
8 \backslash \text { Debug } \backslash \text { source } \backslash \text { DelphiSource } \backslash \text { RulesEditor } \backslash\end{array}$ & 1 & \\
\hline FormRuleEdProjETRec.pas & $\begin{array}{l}\text { C: } \backslash \text { Saphire } \\
8 \backslash \text { Debug } \backslash \text { source } \backslash \text { DelphiSource } \backslash \text { RulesEditor } \backslash\end{array}$ & 1 & \\
\hline FormRuleEdProjFTRec.pas & $\begin{array}{l}\text { C: } \backslash \text { Saphire } \\
8 \backslash \text { Debug } \backslash \text { source } \backslash \text { DelphiSource } \backslash \text { RulesEditor } \backslash\end{array}$ & 1 & \\
\hline FormRuleEdProjPart.pas & $\begin{array}{l}\text { C:\Saphire } \\
8 \backslash \text { Debug } \backslash \text { source } \backslash \text { DelphiSource } \backslash \text { RulesEditor } \backslash\end{array}$ & 1 & \\
\hline FormRuleEdSlice.pas & $\begin{array}{l}\text { C: } \backslash \text { Saphire } \\
8 \backslash \text { Debug } \backslash \text { source } \backslash \text { DelphiSource } \backslash \text { RulesEditor } \backslash\end{array}$ & 1 & \\
\hline FormRuleEdSQPart.pas & $\begin{array}{l}\text { C: } \backslash \text { Saphire } \\
8 \backslash \text { Debug } \backslash \text { source } \backslash \text { DelphiSource } \backslash \text { RulesEditor } \backslash\end{array}$ & 1 & \\
\hline FormRuleEdSQRec.pas & $\begin{array}{l}\text { C: } \backslash \text { Saphire } \\
8 \backslash \text { Debug } \backslash \text { source } \backslash \text { DelphiSource } \backslash \text { RulesEditor } \backslash\end{array}$ & 1 & \\
\hline FormSampleOps.pas & C:\Saphire $8 \backslash$ Debug $\backslash$ source $\backslash$ DelphiSource $\backslash$ & 2 & \\
\hline FormSaveWorkspace.pas & C:\Saphire $8 \backslash$ Debug $\backslash$ source $\backslash$ DelphiSource $\backslash$ & 1 & \\
\hline FormSDP.pas & C:\Saphire $8 \backslash$ Debug $\backslash$ source $\backslash$ DelphiSource $\backslash$ & 4 & \\
\hline FormSDP1.pas & C:\Saphire $8 \backslash$ Debug $\backslash$ source $\backslash$ DelphiSource $\backslash$ & 5 & \\
\hline FormSDP2.pas & C:\Saphire $8 \backslash$ Debug $\backslash$ source $\backslash$ DelphiSource $\backslash$ & 4 & \\
\hline FormSDP3.pas & C:\Saphire $8 \backslash$ Debug $\backslash$ source $\backslash$ DelphiSource $\backslash$ & 3 & \\
\hline FormSDP4.pas & C:\Saphire $8 \backslash$ Debug $\backslash$ source $\backslash$ DelphiSource $\backslash$ & 5 & \\
\hline FormSDPAdvancedRpt.pas & C:\Saphire $8 \backslash$ Debug $\backslash$ source $\backslash$ DelphiSource $\backslash$ & 4 & \\
\hline FormSDPLerfFactors.pas & C:\Saphire $8 \backslash$ Debug $\backslash$ source $\backslash$ DelphiSource $\backslash$ & 4 & \\
\hline
\end{tabular}




\begin{tabular}{|c|c|c|c|}
\hline \multirow[t]{2}{*}{ File Name } & \multirow[t]{2}{*}{ File Path } & \multicolumn{2}{|c|}{$\begin{array}{l}\text { Cyclomatic } \\
\text { Complexity }\end{array}$} \\
\hline & & v8.06.01 & v8.07.17 \\
\hline FormSearch.pas & C:\Saphire 8\Debug $\backslash$ source $\backslash$ DelphiSource $\backslash$ & 4 & \\
\hline FormSearchAdv.pas & C:\Saphire 8\Debug $\backslash$ source $\backslash$ DelphiSource $\backslash$ & 3 & \\
\hline FormSetChangesBase.pas & $\begin{array}{l}\mathrm{C}: \backslash \text { Saphire } \\
8 \backslash \text { Debug } \backslash \text { source } \backslash \text { DelphiSource } \backslash \text { EventEditing } \backslash\end{array}$ & 4 & \\
\hline FormSimulation.pas & C: $\backslash$ Saphire $8 \backslash$ Debug $\backslash$ source $\backslash$ DelphiSource $\backslash$ & 5 & \\
\hline FormSimulationEdit.pas & C:\Saphire 8\Debug $\backslash$ source $\backslash$ DelphiSource $\backslash$ & 4 & \\
\hline FormSimulationEvent.pas & C:\Saphire 8\Debug $\backslash$ source $\backslash$ DelphiSource $\backslash$ & 4 & \\
\hline FormSimulationName.pas & C: $\backslash$ Saphire $8 \backslash$ Debug $\backslash$ source $\backslash$ DelphiSource $\backslash$ & 3 & \\
\hline FormSimulationState.pas & C:\Saphire $8 \backslash$ Debug $\backslash$ source $\backslash$ DelphiSource $\backslash$ & 5 & \\
\hline FormSimulationTrigger.pas & C: $\backslash$ Saphire $8 \backslash$ Debug $\backslash$ source $\backslash$ DelphiSource $\backslash$ & 2 & \\
\hline FormSliceBy.pas & C:\Saphire $8 \backslash$ Debug $\backslash$ source $\backslash$ DelphiSource $\backslash$ & 3 & \\
\hline FormSolveResults.pas & C:\Saphire $8 \backslash$ Debug $\backslash$ source $\backslash$ DelphiSource $\backslash$ & 4 & \\
\hline FormSolveSettings.pas & C:\Saphire $8 \backslash$ Debug $\backslash$ source $\backslash$ DelphiSource $\backslash$ & 4 & \\
\hline FormStartup.pas & C:\Saphire $8 \backslash$ Debug $\backslash$ source $\backslash$ DelphiSource $\backslash$ & 4 & \\
\hline FormStepManager.pas & C: $\backslash$ Saphire $8 \backslash$ Debug $\backslash$ source $\backslash$ DelphiSource $\backslash$ & 3 & \\
\hline FormTemplate.pas & C:\Saphire $8 \backslash$ Debug $\backslash$ source $\backslash$ DelphiSource $\backslash$ & 6 & \\
\hline FormToolBar.pas & C:\Saphire $8 \backslash$ Debug $\backslash$ source $\backslash$ DelphiSource $\backslash$ & 1 & \\
\hline FormUncertainty.pas & C: $\backslash$ Saphire $8 \backslash$ Debug $\backslash$ source $\backslash$ DelphiSource $\backslash$ & 6 & \\
\hline FormUserSetting.pas & C:\Saphire $8 \backslash$ Debug $\backslash$ source $\backslash$ DelphiSource $\backslash$ & 4 & \\
\hline FormViewHtml.pas & C:\Saphire $8 \backslash$ Debug $\backslash$ source $\backslash$ DelphiSource $\backslash$ & 2 & \\
\hline FormViewPdf.pas & C:\Saphire $8 \backslash$ Debug $\backslash$ source $\backslash$ DelphiSource $\backslash$ & 2 & \\
\hline FormWarnSPAR.pas & C: $\backslash$ Saphire $8 \backslash$ Debug $\backslash$ source $\backslash$ DelphiSource $\backslash$ & 2 & \\
\hline FormWManager.pas & $\mathrm{C}: \backslash$ Saphire $8 \backslash$ Debug $\backslash$ source $\backslash$ DelphiSource $\backslash$ & 5 & \\
\hline FormWorkspace.pas & C: $\backslash$ Saphire $8 \backslash$ Debug $\backslash$ source $\backslash$ DelphiSource $\backslash$ & 2 & \\
\hline FormXReference.pas & C:\Saphire $8 \backslash$ Debug $\backslash$ source $\backslash$ DelphiSource $\backslash$ & 9 & \\
\hline FrameDuration.pas & C: $\backslash$ Saphire $8 \backslash$ Debug $\backslash$ source $\backslash$ DelphiSource $\backslash$ & 3 & \\
\hline FrameETGraphicalDisp.pas & $\begin{array}{l}\text { C: } \backslash \text { Saphire } \\
8 \backslash \text { Debug } \backslash \text { source } \backslash \text { DelphiSource } \backslash \text { GraphicalEditi } \\
\text { ng } \backslash\end{array}$ & 6 & \\
\hline FrameFTCSGraphicalDisp.pas & $\begin{array}{l}\text { C: } \backslash \text { Saphire } \\
8 \backslash \text { Debug } \backslash \text { source } \backslash \text { DelphiSource } \backslash \text { GraphicalEditi } \\
\text { ng } \backslash\end{array}$ & 3 & \\
\hline FrameFTGraphicalDisp.pas & $\begin{array}{l}\text { C: } \backslash \text { Saphire } \\
8 \backslash \text { Debug } \backslash \text { source } \backslash \text { DelphiSource } \backslash \text { GraphicalEditi } \\
\text { ng }\end{array}$ & 5 & \\
\hline FrameGraphicalDispBase.pas & $\begin{array}{l}\text { C: } \backslash \text { Saphire } \\
8 \backslash \text { Debug } \backslash \text { source } \backslash \text { DelphiSource } \backslash \text { GraphicalEditi } \\
\text { ng } \backslash\end{array}$ & 4 & \\
\hline FrameHdrModelInfo.pas & C: $\mid$ Saphire $8 \backslash$ Debug $\backslash$ source $\backslash$ DelphiSource $\backslash$ & 4 & \\
\hline
\end{tabular}




\begin{tabular}{|c|c|c|c|}
\hline \multirow[t]{2}{*}{ File Name } & \multirow[t]{2}{*}{ File Path } & \multicolumn{2}{|c|}{$\begin{array}{l}\text { Cyclomatic } \\
\text { Complexity }\end{array}$} \\
\hline & & $\mathbf{v 8 . 0 6 . 0 1}$ & v8.07.17 \\
\hline FrameHdrProjectInfo.pas & C:\Saphire $8 \backslash$ Debug $\backslash$ source $\backslash$ DelphiSource $\backslash$ & 2 & \\
\hline FrameNotes.pas & C:\Saphire $8 \backslash$ Debug $\backslash$ source $\backslash$ DelphiSource $\backslash$ & 2 & \\
\hline frmRulesEditor.pas & $\begin{array}{l}\text { C:\Saphire } \\
8 \backslash \text { Debug } \backslash \text { source } \backslash \text { DelphiSource } \backslash \text { RulesEditor } \backslash\end{array}$ & 3 & \\
\hline FTObject.pas & C:\Saphire $8 \backslash$ Debug $\backslash$ source $\backslash$ DelphiSource $\backslash$ & 2 & \\
\hline FTObject.pas & $\begin{array}{l}\text { C: } \backslash \text { Saphire } \\
8 \backslash \text { Debug } \backslash \text { source } \backslash \text { DelphiSource\_ProjObjects } \backslash\end{array}$ & 3 & \\
\hline FX.pas & $\mathrm{C}: \backslash$ Saphire $8 \backslash$ Debug $\backslash$ source $\backslash$ & 8 & \\
\hline GateObj.pas & $\begin{array}{l}\text { C: } \backslash \text { Saphire } \\
8 \backslash \text { Debug } \backslash \text { source } \backslash \text { DelphiSource } \backslash \text { ProjObjects } \backslash\end{array}$ & 3 & \\
\hline GdiPrint.pas & $\mathrm{C}: \backslash$ Saphire $8 \backslash$ Debug $\backslash$ source $\backslash$ & 10 & \\
\hline GdiUtil.pas & $\mathrm{C}: \backslash$ Saphire $8 \backslash$ Debug $\backslash$ source $\backslash$ & 1 & \\
\hline GdiView.pas & C:\Saphire $8 \backslash$ Debug $\backslash$ source $\backslash$ & 9 & \\
\hline GemH.pas & C:\Saphire $8 \backslash$ Debug $\backslash$ source $\backslash$ DelphiSource $\backslash$ & 0 & \\
\hline GenAn_Editors.pas & C:\Saphire $8 \backslash$ Debug $\backslash$ source $\backslash$ DelphiSource $\backslash$ & 4 & \\
\hline GenAnSupport.pas & $\mathrm{C}: \backslash$ Saphire $8 \backslash$ Debug $\backslash$ source $\backslash$ DelphiSource $\backslash$ & 3 & \\
\hline GIFImage.pas & C:\Saphire $8 \backslash$ Debug $\backslash$ source $\backslash$ DelphiSource $\backslash$ & 4 & \\
\hline GrafDef.pas & C: $\backslash$ Saphire $8 \backslash$ Debug $\backslash$ source $\backslash$ & 0 & \\
\hline Graph2D.pas & C: \Saphire $8 \backslash$ Debug $\backslash$ source $\backslash$ & 6 & \\
\hline GraphicalModelObj.pas & $\begin{array}{l}\text { C: } \backslash \text { Saphire } \\
8 \backslash \text { Debug } \backslash \text { source } \backslash \text { DelphiSource } \backslash \text { GraphicalEditi } \\
n g \backslash\end{array}$ & 4 & \\
\hline GroupData.pas & $\mathrm{C}: \backslash$ Saphire $8 \backslash$ Debug $\backslash$ source $\backslash$ & 6 & \\
\hline HashLib.pas & $\mathrm{C}: \backslash$ Saphire $8 \backslash$ Debug $\backslash$ source $\backslash$ & 5 & \\
\hline HashTable.pas & $\begin{array}{l}\text { C: } \backslash \text { Saphire } \\
8 \backslash \text { Debug } \backslash \text { source } \backslash \text { DelphiSource } \backslash B d d I t e m s \backslash \text { Has } \\
\text { hTableLib } \backslash\end{array}$ & 4 & \\
\hline HelpLib.pas & $\mathrm{C}: \backslash$ Saphire $8 \backslash$ Debug $\backslash$ source $\backslash$ & 1 & \\
\hline HelpTopics.pas & C:\Saphire $8 \backslash$ Debug $\backslash$ source $\backslash$ DelphiSource $\backslash$ & 51 & \\
\hline HRADef.pas & C:\Saphire $8 \backslash$ Debug $\backslash$ source $\backslash$ DelphiSource $\backslash$ & 3 & \\
\hline HRADLL.dpr & C:\Saphire $8 \backslash$ Debug $\backslash$ source $\backslash h f d l l \backslash$ & 5 & \\
\hline HTML.pas & C:\Saphire $8 \backslash$ Debug $\backslash$ source $\backslash$ & 7 & \\
\hline HtmlHelp.pas & $\mathrm{C}: \backslash$ Saphire $8 \backslash$ Debug $\backslash$ source $\backslash$ & 2 & \\
\hline HyperTxt.pas & $\mathrm{C}: \backslash$ Saphire $8 \backslash$ Debug $\backslash$ source $\backslash$ & 10 & \\
\hline ImportCSPathDataObj.pas & $\begin{array}{l}\text { C: } \backslash \text { Saphire } \\
8 \backslash \text { Debug } \backslash \text { source } \backslash \text { DelphiSource } \backslash \text { GraphicalEditi } \\
\text { ng }\end{array}$ & 2 & \\
\hline ImportETDataObject.pas & $\begin{array}{l}\text { C: } \backslash \text { Saphire } \\
8 \backslash \text { Debug } \backslash \text { source } \backslash \text { DelphiSource } \backslash \text { GraphicalEditi }\end{array}$ & 4 & \\
\hline
\end{tabular}




\begin{tabular}{|c|c|c|c|}
\hline \multirow[t]{2}{*}{ File Name } & \multirow[t]{2}{*}{ File Path } & \multicolumn{2}{|c|}{$\begin{array}{l}\text { Cyclomatic } \\
\text { Complexity }\end{array}$} \\
\hline & & v8.06.01 & v8.07.17 \\
\hline & $\mathrm{ng} \backslash$ & & \\
\hline ImportExpBE.pas & $\begin{array}{l}\text { C: } \backslash \text { Saphire } \\
8 \backslash \text { Debug } \backslash \text { source } \backslash \text { DelphiSource } \backslash I m p o r t E x p o r t \backslash\end{array}$ & 6 & \\
\hline ImportFTDataObj.pas & $\begin{array}{l}\text { C: \Saphire } \\
8 \backslash \text { Debug } \backslash \text { source } \backslash \text { DelphiSource } \backslash \text { GraphicalEditi } \\
\text { ng } \backslash\end{array}$ & 4 & \\
\hline InBios.pas & C: $\backslash$ Saphire $8 \backslash$ Debug $\backslash$ source $\backslash$ & 3 & \\
\hline InfoLib.pas & C:\Saphire $8 \backslash$ Debug $\backslash$ source $\backslash$ & 6 & \\
\hline IniDefs.pas & $\mathrm{C}: \backslash$ Saphire $8 \backslash$ Debug $\backslash$ source $\backslash$ DelphiSource $\backslash$ & 3 & \\
\hline Inputs.pas & C: $\backslash$ Saphire $8 \backslash$ Debug $\backslash$ source $\backslash$ & 8 & \\
\hline IOUniversal.pas & C:\Saphire $8 \backslash$ Debug $\backslash$ source $\backslash$ & 3 & \\
\hline LangMap.pas & C: $\backslash$ Saphire $8 \backslash$ Debug $\backslash$ source $\backslash$ & 4 & \\
\hline LibSequence.pas & C:\Saphire $8 \backslash$ Debug $\backslash$ source $\backslash$ & 6 & \\
\hline LinkList.pas & C:\Saphire $8 \backslash$ Debug $\backslash$ source $\backslash$ & 3 & \\
\hline LinkObject.pas & $\mathrm{C}: \backslash$ Saphire $8 \backslash$ Debug $\backslash$ source $\backslash$ & 6 & \\
\hline LinksEd.pas & C:\Saphire $8 \backslash$ Debug $\backslash$ source $\backslash$ & 4 & \\
\hline ListObj.pas & C:\Saphire $8 \backslash$ Debug $\backslash$ source $\backslash$ & 7 & \\
\hline Literal.pas & $\begin{array}{l}\text { C:\Saphire } \\
8 \backslash \text { Debug } \backslash \text { source } \backslash \text { DelphiSource } \backslash \text { PlugIns } \backslash\end{array}$ & 1 & \\
\hline LitTypes.pas & $\begin{array}{l}\text { C:\Saphire } \\
8 \backslash \text { Debug } \backslash \text { source } \backslash \text { DelphiSource } \backslash \text { PlugIns } \backslash\end{array}$ & 0 & \\
\hline Logger.pas & C:\Saphire $8 \backslash$ Debug $\backslash$ source $\backslash$ DelphiSource $\backslash$ & 2 & \\
\hline LogLib.pas & $\mathrm{C}:\lfloor$ Saphire $8 \backslash$ Debug $\backslash$ source $\backslash$ & 9 & \\
\hline LowLongX.pas & C:\Saphire $8 \backslash$ Debug $\backslash$ source $\backslash$ & 4 & \\
\hline MainForm.pas & C:\Saphire $8 \backslash$ Debug $\backslash$ source $\backslash$ schemaObjects $\backslash$ & 3 & \\
\hline Manager.pas & C:\Saphire $8 \backslash$ Debug $\backslash$ source $\backslash$ & 4 & \\
\hline MathL.pas & C:\Saphire $8 \backslash$ Debug $\backslash$ source $\backslash$ & 4 & \\
\hline MathLib.pas & C: \Saphire $8 \backslash$ Debug $\backslash$ source $\backslash$ & 2 & \\
\hline MaxFiles.pas & C:\Saphire $8 \backslash$ Debug $\backslash$ source $\backslash$ & 2 & \\
\hline MBlkEdit.pas & C: $\backslash$ Saphire $8 \backslash$ Debug $\backslash$ source $\backslash$ & 3 & \\
\hline MEditLib.pas & C:\Saphire $8 \backslash$ Debug $\backslash$ source $\backslash$ & 7 & \\
\hline MemCheck.pas & C:\Saphire $8 \backslash$ Debug $\backslash$ source $\backslash$ DelphiSource $\backslash$ & 4 & \\
\hline MenuLib.pas & $\mathrm{C}: \mid$ Saphire $8 \backslash$ Debug $\backslash$ source $\backslash$ & 7 & \\
\hline MergeAPI.pas & C:\Saphire $8 \backslash$ Debug $\backslash$ source $\backslash$ DelphiSource $\backslash$ & 0 & \\
\hline MMTools.pas & C:\Saphire $8 \backslash$ Debug $\backslash$ source $\backslash$ & 2 & \\
\hline ModelData.pas & C:\Saphire $8 \backslash$ Debug $\backslash$ source $\backslash$ DelphiSource $\backslash$ & 7 & \\
\hline ModelData.pas & $\begin{array}{l}\mathrm{C}: \backslash \text { Saphire } \\
8 \backslash \text { Debug } \backslash \text { source } \backslash \text { DelphiSource } \backslash \text { ProjObjects } \backslash\end{array}$ & 4 & \\
\hline
\end{tabular}




\begin{tabular}{|c|c|c|c|}
\hline \multirow[t]{2}{*}{ File Name } & \multirow[t]{2}{*}{ File Path } & \multicolumn{2}{|c|}{$\begin{array}{l}\text { Cyclomatic } \\
\text { Complexity }\end{array}$} \\
\hline & & v8.06.01 & v8.07.17 \\
\hline ModelDataCSTreeNode.pas & $\begin{array}{l}\text { C: } \backslash \text { Saphire } \\
8 \backslash \text { Debug } \backslash \text { source } \backslash \text { DelphiSource } \backslash \text { EventEditing } \backslash\end{array}$ & 3 & \\
\hline ModelDataDefs.pas & $\begin{array}{l}\text { C: } \backslash \text { Saphire } \\
\text { 8\Debug } \backslash \text { source } \backslash \text { DelphiSource } \backslash \text { ProjObjects } \backslash\end{array}$ & 23 & \\
\hline ModelDataTreeNode.pas & $\begin{array}{l}\text { C: } \backslash \text { Saphire } \\
8 \backslash \text { Debug } \backslash \text { source } \backslash \text { DelphiSource } \backslash \text { EventEditing } \backslash\end{array}$ & 6 & \\
\hline ModelLib.pas & C: $\mid$ Saphire $8 \backslash$ Debug $\backslash$ source $\backslash$ DelphiSource $\backslash$ & 3 & \\
\hline ModelTypeObj.pas & C:ISaphire 8\Debug \source\DelphiSource $\backslash$ & 2 & \\
\hline ModSys.pas & C:|Saphire 8\Debug|source\} $&{1} &{ } \\
{\hline \text { MoveLib.pas }} &{\text { C: } \mid \text { Saphire } 8 \backslash \text { Debug } \mid \text { source } \backslash} &{2} &{ } \\
{\hline \text { MSageLib.pas }} &{\text { C:ISaphire 8\Debug|source\} } &{7} &{ } \\
{\hline \text { MsgChk.pas }} &{\text { C:ISaphire 8\Debug } \mid \text { source } \backslash} &{1} &{ } \\
{\hline \text { MsgWrap.pas }} &{\text { C: } \mid \text { Saphire } 8 \backslash \text { Debug } \mid \text { source } \backslash} &{2} &{ } \\
{\hline \text { MultSage.pas }} &{\text { C: } \text { Saphire 8\Debug } \mid \text { source } \backslash} &{5} &{ } \\
{\hline \text { MultUser.pas }} &{\text { C:ISaphire 8\Debug|source\} } &{3} &{ } \\
{\hline \text { MusicLib.pas }} &{\text { C:ISaphire 8\Debug|sourcel }} &{10} &{ } \\
{\hline \text { MynerDFM.pas }} &{\begin{array}{l}\text { C: } \backslash \text { Saphire } \\
8 \backslash \text { Debug } \backslash \text { source } \backslash \text { DelphiSource } \backslash \text { PlugIns } \backslash\end{array}} &{2} &{ } \\
{\hline \text { NewFormFT.pas }} &{\text { C:ISaphire 8\Debug \source\DelphiSource } \backslash} &{2} &{ } \\
{\hline \text { NewProject.pas }} &{\text { C: } \mid \text { Saphire } 8 \backslash \text { Debug } \backslash \text { source } \backslash \text { DelphiSource } \backslash} &{3} &{ } \\
{\hline \text { NewSets.pas }} &{\text { C:ISaphire 8\Debug|source\} } &{3} &{ } \\
{\hline \text { NodePropertyForm.pas }} &{\text { C: } \mid \text { Saphire } 8 \backslash \text { Debug } \backslash \text { source } \backslash \text { DelphiSource } \backslash} &{0} &{ } \\
{\hline \text { NumberIO.pas }} &{\text { C: } \mid \text { Saphire } 8 \backslash \text { Debug } \mid \text { source } \backslash} &{2} &{ } \\
{\hline \text { ObjCalendar.pas }} &{\text { C:ISaphire 8\Debug } \mid \text { source } \backslash} &{7} &{ } \\
{\hline \text { ObjCommon.pas }} &{\text { C:ISaphire 8\Debug } \mid \text { source } \backslash} &{1} &{ } \\
{\hline \text { ObjDef.pas }} &{\text { C: } \mid \text { Saphire } 8 \backslash \text { Debug } \mid \text { source } \backslash} &{1} &{ } \\
{\hline \text { ObjDefault.pas }} &{\text { C:ISaphire 8\Debug } \mid \text { source } \backslash} &{1} &{ } \\
{\hline \text { ObjDialog.pas }} &{\text { C:ISaphire 8\Debug\source } \backslash} &{4} &{ } \\
{\hline \text { ObjEMail.pas }} &{\text { C:ISaphire 8\Debug } \mid \text { source } \backslash} &{7} &{ } \\
{\hline \text { ObjExec.pas }} &{\text { C:|Saphire 8\Debug|source }} &{4} &{ } \\
{\hline \text { ObjExExe.pas }} &{\text { C:ISaphire 8\Debug|source }} &{3} &{ } \\
{\hline \text { ObjExit.pas }} &{\text { C:|Saphire 8\Debug } \mid \text { source } \backslash} &{2} &{ } \\
{\hline \text { ObjFtp.pas }} &{\text { C:|Saphire 8\Debug } \mid \text { source } \backslash} &{5} &{ } \\
{\hline \text { ObjJustOne.pas }} &{\text { C:ISaphire 8\Debug } \mid \text { source } \backslash} &{3} &{ } \\
{\hline \text { ObjLib.pas }} &{\text { C:ISaphire 8\Debug } \mid \text { source } \backslash} &{5} &{ } \\
{\hline \text { ObjMenu.pas }} &{\text { C:ISaphire 8\Debug } \mid \text { source } \backslash} &{8} &{ } \\
{\hline \text { ObjMessage.pas }} &{\text { C:ISaphire 8\Debug|source }} &{4} &{ } \\
\hline\end{array}$
\end{tabular}

Page 21 


\begin{tabular}{|c|c|c|c|}
\hline \multirow[t]{2}{*}{ File Name } & \multirow[t]{2}{*}{ File Path } & \multicolumn{2}{|c|}{$\begin{array}{l}\text { Cyclomatic } \\
\text { Complexity }\end{array}$} \\
\hline & & v8.06.01 & v8.07.17 \\
\hline ObjPool.pas & C:|Saphire $8 \backslash$ Debug $\backslash$ source $\backslash$ & 4 & \\
\hline ObjPropSheet.pas & C: $\mid$ Saphire $8 \backslash$ Debug $\mid$ source $\backslash$ & 5 & \\
\hline ObjSage.pas & C:|Saphire $8 \backslash$ Debug $\backslash$ source $\backslash$ & 2 & \\
\hline ObjSequence.pas & C: $\mid$ Saphire $8 \backslash$ Debug $\mid$ source $\backslash$ & 2 & \\
\hline ObjSplash.pas & C:|Saphire 8\Debug|source $\backslash$ & 4 & \\
\hline ObjWindow.pas & C:|Saphire 8\Debug|source $\backslash$ & 2 & \\
\hline Parser.pas & $\begin{array}{l}\text { C: } \backslash \text { Saphire } \\
\text { 8\Debug } \backslash \text { source } \backslash \text { DelphiSource } \backslash \text { RulesEditor } \backslash\end{array}$ & 19 & \\
\hline ParseRtf.pas & C: \Saphire 8\Debug \source \DelphiSource $\backslash$ & 9 & \\
\hline PCXImage.pas & C:|Saphire 8\Debug|source $\backslash$ & 1 & \\
\hline PidDir2Xml.pas & C: $\mid$ Saphire $8 \backslash$ Debug $\backslash$ source $\backslash$ DelphiSource $\backslash$ & 6 & \\
\hline PlugCCFStag.dpr & $\begin{array}{l}\text { C: } \backslash \text { Saphire } \\
\text { 8\Debug } \backslash \text { source } \backslash \text { DelphiSource } \backslash \text { PlugIns } \backslash\end{array}$ & 2 & \\
\hline PlugCCFUtil.pas & $\begin{array}{l}\text { C: } \backslash \text { Saphire } \\
\text { 8\Debug } \backslash \text { source } \backslash \text { DelphiSource } \backslash \text { PlugIns } \backslash\end{array}$ & 20 & \\
\hline PlugDef.pas & $\begin{array}{l}\text { C: } \backslash \text { Saphire } \\
8 \backslash \text { Debug } \backslash \text { source } \backslash \text { DelphiSource } \backslash \text { PlugIns } \backslash\end{array}$ & 0 & \\
\hline PlugDFM.dpr & $\begin{array}{l}\text { C: } \backslash \text { Saphire } \\
\text { 8\Debug } \backslash \text { source } \backslash \text { DelphiSource } \backslash \text { PlugIns } \backslash\end{array}$ & 2 & \\
\hline PntrDev.pas & C:|Saphire 8\Debug|source $\backslash$ & 1 & \\
\hline Pool.pas & C:ISaphire 8\Debug|source\} $&{6} &{ } \\
{\hline \text { Profile.pas }} &{\text { C:|Saphire 8\Debug|source } \backslash} &{4} &{ } \\
{\hline \text { ProgCmnd.pas }} &{\text { C: } \mid \text { Saphire } 8 \backslash \text { Debug } \mid \text { source } \backslash} &{4} &{ } \\
{\hline \text { ProgramX.pas }} &{\text { C: } \mid \text { Saphire } 8 \backslash \text { Debug } \mid \text { source } \backslash} &{2} &{ } \\
{\hline \text { ProjChanges.pas }} &{\text { C:ISaphire 8\Debug \source\DelphiSource } \backslash} &{2} &{ } \\
{\hline \text { Project_Object.pas }} &{\text { C:ISaphire 8\Debug \source \DelphiSource } \backslash} &{4} &{ } \\
{\hline \text { Publish.pas }} &{\text { C: } \mid \text { Saphire } 8 \backslash \text { Debug } \backslash \text { source } \backslash \text { DelphiSource } \backslash} &{3} &{ } \\
{\hline \text { Qualify.pas }} &{\text { C: } \mid \text { Saphire } 8 \backslash \text { Debug } \mid \text { source } \backslash} &{9} &{ } \\
{\hline \text { QueryAux.pas }} &{\text { C:|Saphire 8\Debug|source\} } &{9} &{ } \\
{\hline \text { QueryLib.pas }} &{\text { C:|Saphire } 8 \backslash \text { Debug|source\} } &{9} &{ } \\
{\hline \text { QuickBE.pas }} &{\text { C: \Saphire 8\Debug \source\DelphiSource } \backslash} &{2} &{ } \\
{\hline \text { QuickEditObj.pas }} &{\begin{array}{l}\text { C: } \mid \text { Saphire } \\
8 \backslash \text { Debug } \backslash \text { source } \backslash \text { DelphiSource } \backslash \text { GraphicalEditi } \\
\text { ng } \backslash\end{array}} &{6} &{ } \\
{\hline \text { RANCACHE.pas }} &{\text { C: } \mid \text { Saphire } 8 \backslash \text { Debug } \mid \text { source } \backslash} &{6} &{ } \\
{\hline \text { Random.pas }} &{\text { C:|Saphire 8\Debug|source\} } &{2} &{ } \\
{\hline \text { RaspCCFObj.pas }} &{\text { C: \Saphire } 8 \backslash \text { Debug } \backslash \text { source } \backslash \text { DelphiSource } \backslash} &{4} &{ } \\
{\hline \text { RConversions.pas }} &{\text { C:|Saphire 8\Debug|source\} } &{7} &{ } \\
\hline\end{array}$
\end{tabular}

Page 22 


\begin{tabular}{|c|c|c|c|}
\hline \multirow[t]{2}{*}{ File Name } & \multirow[t]{2}{*}{ File Path } & \multicolumn{2}{|c|}{$\begin{array}{l}\text { Cyclomatic } \\
\text { Complexity }\end{array}$} \\
\hline & & v8.06.01 & v8.07.17 \\
\hline ReliabilityBlockObject.pas & C: $\mid$ Saphire $8 \backslash$ Debug $\backslash$ source $\backslash$ DelphiSource $\backslash$ & 2 & \\
\hline RelLock.pas & C:|Saphire $8 \backslash$ Debug $\mid$ source $\backslash$ & 5 & \\
\hline Reports.pas & C:|Saphire 8\Debug|source\} $&{5} &{ } \\
{\hline \text { ResDir.pas }} &{\text { C:|Saphire 8\Debug|source\} } &{4} &{ } \\
{\hline \text { ResLib.pas }} &{\text { C:|Saphire 8\Debug|source } \backslash} &{12} &{ } \\
{\hline \text { Resource.pas }} &{\text { C:|Saphire 8\Debug \source } \backslash} &{5} &{ } \\
{\hline \text { RptCutSets.pas }} &{\text { C:ISaphire 8\Debug \source \DelphiSource } \backslash} &{5} &{ } \\
{\hline \text { RptGraphics.pas }} &{\text { C: \Saphire 8\Debug \source \DelphiSource } \backslash} &{5} &{ } \\
{\hline \text { RptGroups.pas }} &{\text { C: \Saphire 8\Debug \source\DelphiSource } \backslash} &{4} &{ } \\
{\hline \text { RptImportance.pas }} &{\text { C: } \mid \text { Saphire } 8 \backslash \text { Debug } \backslash \text { source } \backslash \text { DelphiSource } \backslash} &{3} &{ } \\
{\hline \text { RptObjs.pas }} &{\text { C: \Saphire 8\Debug \source \DelphiSource } \backslash} &{4} &{ } \\
{\hline \text { RptTemplate.pas }} &{\text { C:ISaphire 8\Debug \source \DelphiSource } \backslash} &{2} &{ } \\
{\hline \text { RTF.pas }} &{\text { C: \Saphire 8\Debug|source\} } &{11} &{ } \\
{\hline \text { RulesItem.pas }} &{\begin{array}{l}\text { C: } \backslash \text { Saphire } \\
\text { 8\Debug } \backslash \text { source } \backslash \text { DelphiSource } \backslash \text { RulesEditor } \backslash\end{array}} &{4} &{ } \\
{\hline \text { RulesSchema.pas }} &{\begin{array}{l}\text { C: } \backslash \text { Saphire } \\
\text { 8\Debug } \backslash \text { source } \backslash \text { DelphiSource } \backslash \text { RulesEditor } \backslash\end{array}} &{1} &{ } \\
{\hline \text { RulesUtil.pas }} &{\text { C:ISaphire 8\Debug \source \DelphiSource } \backslash} &{14} &{ } \\
{\hline \text { RunProgX.pas }} &{\text { C: } \mid \text { Saphire } 8 \backslash \text { Debug } \mid \text { source } \backslash} &{4} &{ } \\
{\hline \text { Sage.pas }} &{\text { C:|Saphire 8\Debug|source }} &{4} &{ } \\
{\hline \text { SageBttn.pas }} &{\text { C:ISaphire 8\Debug|source }} &{10} &{ } \\
{\hline \text { SageCBox.pas }} &{\text { C:|Saphire 8\Debug|source\} } &{11} &{ } \\
{\hline \text { SageCC.pas }} &{\text { C: } \mid \text { Saphire } 8 \backslash \text { Debug } \mid \text { source } \backslash} &{0} &{ } \\
{\hline \text { SageComb.pas }} &{\text { C:|Saphire 8\Debug|source } \backslash} &{14} &{ } \\
{\hline \text { SageDialogs.pas }} &{\text { C:|Saphire } 8 \backslash \text { Debug } \mid \text { source } \backslash} &{9} &{ } \\
{\hline \text { SageDyn.pas }} &{\text { C:|Saphire 8\Debug \source } \backslash} &{7} &{ } \\
{\hline \text { SageEdit.pas }} &{\text { C:ISaphire 8\Debug|source }} &{16} &{ } \\
{\hline \text { SageErrs.pas }} &{\text { C:|Saphire 8\Debug \source } \backslash} &{2} &{ } \\
{\hline \text { SageFinal.pas }} &{\text { C:ISaphire 8\Debug|source } \backslash} &{1} &{ } \\
{\hline \text { SageGrid.pas }} &{\text { C:|Saphire 8\Debug|source\} } &{9} &{ } \\
{\hline \text { SageInit.pas }} &{\text { C:|Saphire } 8 \backslash \text { Debug } \backslash \text { source } \backslash} &{1} &{ } \\
{\hline \text { SageKey.pas }} &{\text { C:ISaphire 8\Debug|source\} } &{7} &{ } \\
{\hline \text { SageLib.pas }} &{\text { C:|Saphire 8\Debug|source } \backslash} &{7} &{ } \\
{\hline \text { SageList.pas }} &{\text { C:ISaphire 8\Debug|source }} &{9} &{ } \\
{\hline \text { SageLV.pas }} &{\text { C:|Saphire 8\Debug|source } \backslash} &{6} &{ } \\
{\hline \text { SageMask.pas }} &{\text { C:|Saphire } 8 \backslash \text { Debug } \backslash \text { source } \backslash} &{11} &{ } \\
\hline\end{array}$
\end{tabular}

Page 23 


\begin{tabular}{|c|c|c|c|}
\hline \multirow[t]{2}{*}{ File Name } & \multirow[t]{2}{*}{ File Path } & \multicolumn{2}{|c|}{$\begin{array}{l}\text { Cyclomatic } \\
\text { Complexity }\end{array}$} \\
\hline & & v8.06.01 & v8.07.17 \\
\hline SageMenu.pas & C:|Saphire 8\Debug|source\} $&{7} &{ } \\
{\hline \text { SageOpt.pas }} &{\text { C: } \mid \text { Saphire } 8 \backslash \text { Debug } \mid \text { source } \backslash} &{11} &{ } \\
{\hline \text { SageRBtn.pas }} &{\text { C:|Saphire 8\Debug|source\} } &{10} &{ } \\
{\hline \text { SageRich.pas }} &{\text { C:ISaphire 8\Debug|source\} } &{11} &{ } \\
{\hline \text { SageRisk2.pas }} &{\text { C: \Saphire 8\Debug \source \DelphiSource } \backslash} &{2} &{ } \\
{\hline \text { SageRiskUtil.pas }} &{\text { C: \Saphire 8\Debug \source\DelphiSource } \backslash} &{5} &{ } \\
{\hline \text { SageSBar.pas }} &{\text { C:|Saphire 8\Debug|source\} } &{7} &{ } \\
{\hline \text { SageSpec.pas }} &{\text { C: } \mid \text { Saphire } 8 \backslash \text { Debug } \mid \text { source } \backslash} &{2} &{ } \\
{\hline \text { SageSplash.pas }} &{\text { C:ISaphire 8\Debug|source\} } &{2} &{ } \\
{\hline \text { SageST.dpr }} &{\text { C:|Saphire } 8 \backslash \text { Debug } \mid \text { source } \backslash} &{1} &{ } \\
{\hline \text { SageStat.pas }} &{\text { C:\Saphire 8\Debug|source\} } &{8} &{ } \\
{\hline \text { SageSTDataSet.pas }} &{\text { C:|Saphire 8\Debug } \mid \text { source } \backslash} &{3} &{ } \\
{\hline \text { SageSTObject.pas }} &{\text { C: \Saphire 8\Debug|source\} } &{2} &{ } \\
{\hline \text { SageSTPackage.dpk }} &{\text { C:|Saphire 8\Debug|source\} } &{0} &{ } \\
{\hline \text { SageSTSchemaObjects.dpr }} &{\text { C: } \mid \text { Saphire } 8 \backslash \text { Debug } \backslash \text { source } \backslash \text { schemaObjects } \backslash} &{1} &{ } \\
{\hline \text { SageTerm.pas }} &{\text { C:|Saphire 8\Debug|source\} } &{4} &{ } \\
{\hline \text { SageTree.pas }} &{\text { C:|Saphire 8\Debug|source\} } &{14} &{ } \\
{\hline \text { SaphCache.pas }} &{\text { C: \Saphire } 8 \backslash \text { Debug } \backslash \text { source } \backslash \text { DelphiSource } \backslash} &{2} &{ } \\
{\hline \text { SaphDataM.pas }} &{\text { C:ISaphire 8\Debug \source\DelphiSource } \backslash} &{0} &{ } \\
{\hline \text { SAPHIRE.dpr }} &{\text { C: \Saphire 8\Debug \source \DelphiSource } \backslash} &{5} &{ } \\
{\hline \text { SAPHIRE_Project.dpr }} &{\text { C: \Saphire 8\Debug \source \DelphiSource } \backslash} &{9} &{ } \\
{\hline \text { SAPHIRE8.pas }} &{\text { C: \Saphire 8\Debug \source\DelphiSource } \backslash} &{3} &{ } \\
{\hline \text { SaphireAnalysis.pas }} &{\text { C: \Saphire } 8 \backslash \text { Debug } \backslash \text { source } \backslash \text { DelphiSource } \backslash} &{5} &{ } \\
{\hline \text { saphmacro.pas }} &{\text { C: } \mid \text { Saphire } 8 \backslash \text { Debug } \backslash \text { source } \backslash \text { DelphiSource } \backslash} &{24} &{ } \\
{\hline \text { SaphSet.pas }} &{\text { C: \Saphire 8\Debug \source\DelphiSource } \backslash} &{2} &{ } \\
{\hline \text { ScanLib.pas }} &{\text { C:ISaphire 8\Debug|source\} } &{1} &{ } \\
{\hline \text { Scroll.pas }} &{\text { C:|Saphire 8\Debug|source\} } &{9} &{ } \\
{\hline \text { SDP_Editors.pas }} &{\text { C: } \backslash \text { Saphire } 8 \backslash \text { Debug } \backslash \text { source } \backslash \text { DelphiSource } \backslash} &{4} &{ } \\
{\hline \text { SDP_Insights.pas }} &{\text { C:ISaphire 8\Debug \source \DelphiSource } \backslash} &{4} &{ } \\
{\hline \text { SDPReports.pas }} &{\text { C: \Saphire 8\Debug \source\DelphiSource } \backslash} &{5} &{ } \\
{\hline \text { SearchObj.pas }} &{\text { C: } \mid \text { Saphire } 8 \backslash \text { Debug } \backslash \text { source } \backslash \text { DelphiSource } \backslash} &{4} &{ } \\
{\hline \text { SecurLib.pas }} &{\text { C:ISaphire 8\Debug|source\} } &{3} &{ } \\
{\hline \text { Select.pas }} &{\text { C:|Saphire 8\Debug|source\} } &{2} &{ } \\
{\hline \text { SelectModel.pas }} &{\text { C: \Saphire 8\Debug \source \DelphiSource } \backslash} &{2} &{ } \\
{\hline \text { Semaphore.pas }} &{\text { C: } \mid \text { Saphire } 8 \backslash \text { Debug } \mid \text { source } \backslash} &{4} &{ } \\
\hline\end{array}$
\end{tabular}




\begin{tabular}{|c|c|c|c|}
\hline \multirow[t]{2}{*}{ File Name } & \multirow[t]{2}{*}{ File Path } & \multicolumn{2}{|c|}{$\begin{array}{l}\text { Cyclomatic } \\
\text { Complexity }\end{array}$} \\
\hline & & v8.06.01 & v8.07.17 \\
\hline SetLib.pas & C:|Saphire 8\Debug|source $\backslash$ & 3 & \\
\hline SGK.pas & C: $\mid$ Saphire $8 \backslash$ Debug $\mid$ source $\backslash$ & 5 & \\
\hline SGKDevice.pas & C: $\backslash$ Saphire $8 \backslash$ Debug $\backslash$ source $\backslash$ & 0 & \\
\hline SGKDriver.pas & C: $\mid$ Saphire $8 \backslash$ Debug $\mid$ source $\backslash$ & 3 & \\
\hline SGKErrors.pas & C:\Saphire $8 \backslash$ Debug $\backslash$ source $\backslash$ & 2 & \\
\hline SGKFill.pas & C:|Saphire 8\Debug|source $\backslash$ & 11 & \\
\hline SgkFinal.pas & C:\Saphire $8 \backslash$ Debug $\backslash$ source $\backslash$ & 1 & \\
\hline SGKFont.pas & C:\Saphire $8 \backslash$ Debug $\backslash$ source $\backslash$ & 6 & \\
\hline SGKGraphics.pas & C:|Saphire $8 \backslash$ Debug $\backslash$ source $\backslash$ & 4 & \\
\hline SGKInBios.pas & C:\Saphire 8\Debug $\backslash$ source $\backslash$ & 3 & \\
\hline SgkInit.pas & C: $\mid$ Saphire $8 \backslash$ Debug $\backslash$ source $\backslash$ & 1 & \\
\hline SGKInputs.pas & C:\Saphire 8\Debug $\backslash$ source $\backslash$ & 8 & \\
\hline SGKLine.pas & C:\Saphire $8 \backslash$ Debug $\backslash$ source $\backslash$ & 8 & \\
\hline SGKMarker.pas & C:\Saphire $8 \backslash$ Debug $\backslash$ source $\backslash$ & 2 & \\
\hline SGKPCX.pas & C:\Saphire $8 \backslash$ Debug $\backslash$ source $\backslash$ & 1 & \\
\hline SGKSupport.pas & C:|Saphire $8 \backslash$ Debug $\backslash$ source $\backslash$ & 1 & \\
\hline SGKUtil.pas & C:\Saphire $8 \backslash$ Debug $\backslash$ source $\backslash$ & 5 & \\
\hline SGKWin.pas & C:|Saphire 8\Debug \source\} $&{4} &{ } \\
{\hline \text { SGMenu.pas }} &{\text { C:\Saphire 8\Debug } \backslash \text { source } \backslash} &{7} &{ } \\
{\hline \text { Simulation_Main.pas }} &{\text { C: } \mid \text { Saphire } 8 \backslash \text { Debug } \backslash \text { source } \backslash \text { DelphiSource } \backslash} &{4} &{ } \\
{\hline \text { SimulationBaseObject.pas }} &{\text { C:ISaphire } 8 \backslash \text { Debug } \backslash \text { source } \backslash \text { DelphiSource } \backslash} &{3} &{ } \\
{\hline \text { SimulationEdit_Main.pas }} &{\text { C: } \mid \text { Saphire } 8 \backslash \text { Debug } \backslash \text { source } \backslash \text { DelphiSource } \backslash} &{5} &{ } \\
{\hline \text { SimulationObject.pas }} &{\text { C: } \backslash \text { Saphire } 8 \backslash \text { Debug } \backslash \text { source } \backslash \text { DelphiSource } \backslash} &{3} &{ } \\
{\hline \text { SortLibX.pas }} &{\text { C: } \mid \text { Saphire } 8 \backslash \text { Debug } \backslash \text { source } \backslash} &{4} &{ } \\
{\hline \text { SortList.pas }} &{\text { C:\Saphire 8\Debug } \backslash \text { source } \backslash} &{8} &{ } \\
{\hline \text { SortMrg.pas }} &{\text { C: } \mid \text { Saphire } 8 \backslash \text { Debug } \backslash \text { source } \backslash} &{6} &{ } \\
{\hline \text { Soundex.pas }} &{\text { C:\Saphire } 8 \backslash \text { Debug } \backslash \text { source } \backslash} &{11} &{ } \\
{\hline \text { Sounds.pas }} &{\text { C:\Saphire 8\Debug } \backslash \text { source } \backslash} &{3} &{ } \\
{\hline \text { SPARH.pas }} &{\text { C: } \mid \text { Saphire } 8 \backslash \text { Debug } \backslash \text { source } \backslash \text { DelphiSource } \backslash} &{7} &{ } \\
{\hline \text { SPARH.pas }} &{\text { C: \Saphire 8\Debug } \backslash \text { source } \backslash \text { hfdll } \backslash} &{7} &{ } \\
{\hline \text { SparRpt.pas }} &{\text { C: } \mid \text { Saphire } 8 \backslash \text { Debug } \backslash \text { source } \backslash \text { DelphiSource } \backslash} &{4} &{ } \\
{\hline \text { SPDLib.pas }} &{\text { C:\Saphire 8\Debug } \backslash \text { source } \backslash} &{6} &{ } \\
{\hline \text { SplashScreenForm.pas }} &{\text { C: \Saphire } 8 \backslash \text { Debug } \backslash \text { source } \backslash \text { DelphiSource } \backslash} &{1} &{ } \\
{\hline \text { SqCanvas.pas }} &{\text { C: } \backslash \text { Saphire } 8 \backslash \text { Debug } \backslash \text { source } \backslash} &{8} &{ } \\
{\hline \text { SqCheck.pas }} &{\text { C:|Saphire } 8 \backslash \text { Debug } \backslash \text { source } \backslash} &{5} &{ } \\
$\hline
\end{tabular}




\begin{tabular}{|c|c|c|c|}
\hline \multirow[t]{2}{*}{ File Name } & \multirow[t]{2}{*}{ File Path } & \multicolumn{2}{|c|}{$\begin{array}{l}\text { Cyclomatic } \\
\text { Complexity }\end{array}$} \\
\hline & & v8.06.01 & v8.07.17 \\
\hline SqDef.pas & C:|Saphire 8\Debug|source\} $&{0} &{ } \\
{\hline \text { SqEdDef.pas }} &{\text { C:ISaphire 8\Debug|source }} &{0} &{ } \\
{\hline \text { SqEdit.pas }} &{\text { C:|Saphire 8\Debug|source }} &{6} &{ } \\
{\hline \text { SqExec.pas }} &{\text { C:ISaphire 8\Debug } \mid \text { source } \backslash} &{6} &{ } \\
{\hline \text { SqGShow.pas }} &{\text { C:|Saphire 8\Debug \source } \backslash} &{3} &{ } \\
{\hline \text { SqGSmbl.pas }} &{\text { C:ISaphire 8\Debug|source }} &{4} &{ } \\
{\hline \text { SqGTop.pas }} &{\text { C:ISaphire 8\Debug|source }} &{1} &{ } \\
{\hline \text { SqGUtil.pas }} &{\text { C:|Saphire } 8 \backslash \text { Debug } \mid \text { source } \backslash} &{3} &{ } \\
{\hline \text { SQLAct.pas }} &{\text { C:ISaphire 8\Debug|source\} } &{25} &{ } \\
{\hline \text { SQLCheck.pas }} &{\text { C:|Saphire } 8 \backslash \text { Debug } \mid \text { source } \backslash} &{6} &{ } \\
{\hline \text { SQLExec.pas }} &{\text { C: } \mid \text { Saphire } 8 \backslash \text { Debug } \backslash \text { source } \backslash} &{4} &{ } \\
{\hline \text { SqLinks.pas }} &{\text { C:|Saphire 8\Debug|source\} } &{4} &{ } \\
{\hline \text { SQLParse.pas }} &{\text { C:\Saphire 8\Debug|source }} &{19} &{ } \\
{\hline \text { SQLRun.pas }} &{\text { C:ISaphire 8\Debug|source\} } &{8} &{ } \\
{\hline \text { SQLScan.pas }} &{\text { C:|Saphire 8\Debug|source }} &{5} &{ } \\
{\hline \text { SQLUtil.pas }} &{\text { C:ISaphire 8\Debug|source }} &{6} &{ } \\
{\hline \text { SqOccur.pas }} &{\text { C: } \mid \text { Saphire } 8 \backslash \text { Debug } \mid \text { source } \backslash} &{5} &{ } \\
{\hline \text { SqUtil.pas }} &{\text { C:|Saphire 8\Debug|source } \backslash} &{4} &{ } \\
{\hline \text { STBackup.pas }} &{\text { C:ISaphire 8\Debug|source }} &{3} &{ } \\
{\hline \text { STCustomDataSet.pas }} &{\text { C:|Saphire 8\Debug|source\} } &{2} &{ } \\
{\hline \text { STNative.pas }} &{\text { C:|Saphire } 8 \backslash \text { Debug } \backslash \text { source } \backslash} &{3} &{ } \\
{\hline \text { StorageX.pas }} &{\text { C:|Saphire 8\Debug|source }} &{2} &{ } \\
{\hline \text { STRegistry.pas }} &{\text { C:|Saphire 8\Debug|source } \backslash} &{3} &{ } \\
{\hline \text { STResourceObjects.pas }} &{\text { C:ISaphire 8\Debug|source\} } &{4} &{ } \\
{\hline \text { StringGridLib.pas }} &{\text { C:|Saphire } 8 \backslash \text { Debug } \mid \text { source } \backslash} &{5} &{ } \\
{\hline \text { StringsX.pas }} &{\text { C:ISaphire 8\Debug|source }} &{4} &{ } \\
{\hline \text { StrokeFont.pas }} &{\text { C:|Saphire 8\Debug|source\} } &{5} &{ } \\
{\hline \text { STSecurity.pas }} &{\text { C:ISaphire 8\Debug|source\} } &{9} &{ } \\
{\hline \text { SubUnorderedBDD.pas }} &{\begin{array}{l}\text { C: } \backslash \text { Saphire } \\
8 \backslash \text { Debug } \backslash \text { source } \backslash \text { DelphiSource } \backslash \text { BddItems } \backslash\end{array}} &{4} &{ } \\
{\hline \text { SuperIO.pas }} &{\text { C:ISaphire 8\Debug } \mid \text { source } \backslash} &{7} &{ } \\
{\hline \text { Supermsk.pas }} &{\text { C: \Saphire 8\Debug \source \DelphiSource } \backslash} &{18} &{ } \\
{\hline \text { SymParse.pas }} &{\text { C:ISaphire 8\Debug|source\} } &{6} &{ } \\
{\hline \text { SynEdit.pas }} &{\begin{array}{l}\text { C: } \backslash \text { Saphire } \\
\text { 8\Debug } \backslash \text { source } \backslash \text { DelphiSource } \backslash \text { RulesEditor } \backslash\end{array}} &{6} &{ } \\
{\hline \text { SynHighlighterMacMan.pas }} &{\text { C:\Saphire 8\Debug \source \DelphiSource } \backslash} &{3} &{ } \\
\hline\end{array}$
\end{tabular}




\begin{tabular}{|c|c|c|c|}
\hline \multirow[t]{2}{*}{ File Name } & \multirow[t]{2}{*}{ File Path } & \multicolumn{2}{|c|}{$\begin{array}{l}\text { Cyclomatic } \\
\text { Complexity }\end{array}$} \\
\hline & & v8.06.01 & v8.07.17 \\
\hline SynHighlighterSaphL.pas & $\begin{array}{l}\text { C: } \backslash \text { Saphire } \\
\text { 8\Debug } \backslash \text { source } \backslash \text { DelphiSource } \backslash \text { RulesEditor } \backslash\end{array}$ & 3 & \\
\hline SynHighlighterSaphP.pas & $\begin{array}{l}\text { C: } \mid \text { Saphire } \\
\text { 8\Debug } \backslash \text { source } \backslash \text { DelphiSource } \backslash \text { RulesEditor } \backslash\end{array}$ & 3 & \\
\hline SynHighlighterSaphR.pas & $\begin{array}{l}\text { C: } \backslash \text { Saphire } \\
\text { 8\Debug } \backslash \text { source } \backslash \text { DelphiSource } \backslash \text { RulesEditor } \backslash\end{array}$ & 3 & \\
\hline SynHighlighterSaphS.pas & $\begin{array}{l}\text { C: } \mid \text { Saphire } \\
\text { 8\Debug } \backslash \text { source } \backslash \text { DelphiSource } \backslash \text { RulesEditor } \backslash\end{array}$ & 3 & \\
\hline TermX.pas & C:\Saphire 8\Debug|source $\backslash$ & 4 & \\
\hline TextBase.pas & C:|Saphire 8\Debug|source\} $&{10} &{ } \\
{\hline \text { TextDB.pas }} &{\text { C:|Saphire 8\Debug \source } \backslash} &{5} &{ } \\
{\hline \text { TextEd2.pas }} &{\text { C:|Saphire 8\Debug|source\} } &{12} &{ } \\
{\hline \text { TextEdit.pas }} &{\text { C:\Saphire 8\Debug|source \} } &{6} &{ } \\
{\hline \text { TextShow.pas }} &{\text { C:ISaphire } 8 \backslash \text { Debug } \mid \text { source } \backslash} &{10} &{ } \\
{\hline \text { TextX.pas }} &{\text { C:\Saphire } 8 \backslash \text { Debug } \backslash \text { source } \backslash} &{4} &{ } \\
{\hline \text { ThorAdj.pas }} &{\text { C:|Saphire 8\Debug|source\} } &{12} &{ } \\
{\hline \text { ThorBld.pas }} &{\text { C:|Saphire 8\Debug|source\} } &{9} &{ } \\
{\hline \text { ThorEdit.pas }} &{\text { C:|Saphire } 8 \backslash \text { Debug } \mid \text { source } \backslash} &{11} &{ } \\
{\hline \text { ThorForm.pas }} &{\text { C: } \mid \text { Saphire } 8 \backslash \text { Debug } \mid \text { source } \backslash} &{7} &{ } \\
{\hline \text { ThorGraf.pas }} &{\text { C: } \backslash \text { Saphire } 8 \backslash \text { Debug } \backslash \text { source } \backslash} &{7} &{ } \\
{\hline \text { ThorLib.pas }} &{\text { C:|Saphire 8\Debug|source\} } &{9} &{ } \\
{\hline \text { ThorLibX.pas }} &{\text { C:\Saphire } 8 \backslash \text { Debug } \backslash \text { source } \backslash} &{8} &{ } \\
{\hline \text { ThorLoad.pas }} &{\text { C: } \mid \text { Saphire } 8 \backslash \text { Debug } \mid \text { source } \backslash} &{8} &{ } \\
{\hline \text { ThorPort.pas }} &{\text { C:ISaphire 8\Debug|source\} } &{3} &{ } \\
{\hline \text { ThorSer.pas }} &{\text { C:|Saphire 8\Debug|source\} } &{6} &{ } \\
{\hline \text { ThorShow.pas }} &{\text { C:|Saphire 8\Debug|source\} } &{8} &{ } \\
{\hline \text { ThorSpec.pas }} &{\text { C:|Saphire 8\Debug|source\} } &{1} &{ } \\
{\hline \text { ThorUtil.pas }} &{\text { C:|Saphire 8\Debug|source\} } &{1} &{ } \\
{\hline \text { TimeDate.pas }} &{\text { C:|Saphire 8\Debug|source\} } &{4} &{ } \\
{\hline \text { TimeLib.pas }} &{\text { C:|Saphire 8\Debug|source\} } &{5} &{ } \\
{\hline \text { TimeReal.pas }} &{\text { C:ISaphire 8\Debug|source }} &{3} &{ } \\
{\hline \text { TokenLib.pas }} &{\text { C:|Saphire 8\Debug|source } \backslash} &{3} &{ } \\
{\hline \text { Toolbar.pas }} &{\text { C:ISaphire 8\Debug|source\} } &{10} &{ } \\
{\hline \text { TPCVideo.pas }} &{\text { C:|Saphire 8\Debug|source\} } &{5} &{ } \\
{\hline \text { Trackit.pas }} &{\text { C: } \mid \text { Saphire } 8 \backslash \text { Debug } \mid \text { source } \backslash} &{3} &{ } \\
{\hline \text { Transact.pas }} &{\text { C:|Saphire 8\Debug|source }} &{6} &{ } \\
{\hline \text { TreeRiskAssessment.pas }} &{\text { C:ISaphire }} &{3} &{ } \\
\hline\end{array}$
\end{tabular}




\begin{tabular}{|c|c|c|c|}
\hline \multirow[t]{2}{*}{ File Name } & \multirow[t]{2}{*}{ File Path } & \multicolumn{2}{|c|}{$\begin{array}{l}\text { Cyclomatic } \\
\text { Complexity }\end{array}$} \\
\hline & & v8.06.01 & v8.07.17 \\
\hline & $\begin{array}{l}\text { 8\Debug } \backslash \text { source } \backslash \text { DelphiSource } \backslash \text { GraphicalEditi } \\
\text { ng } \backslash\end{array}$ & & \\
\hline TrueTypeFont.pas & C:|Saphire $8 \backslash$ Debug $\mid$ source $\backslash$ & 3 & \\
\hline TxtBLib.pas & C:|Saphire 8\Debug|source\} $&{9} &{ } \\
{\hline \text { UI_Utils.pas }} &{\text { C:ISaphire 8\Debug \source \DelphiSource } \backslash} &{9} &{ } \\
{\hline \text { Uncertainty.pas }} &{\text { C:ISaphire 8\Debug \source \DelphiSource } \backslash} &{0} &{ } \\
{\hline \text { UncertAreaChart.pas }} &{\text { C:ISaphire 8\Debug \source \DelphiSource } \backslash} &{3} &{ } \\
{\hline \text { UniqueID.pas }} &{\begin{array}{l}\text { C: } \mid \text { Saphire } \\
8 \backslash \text { Debug } \backslash \text { source } \backslash \text { DelphiSource } \backslash \text { GraphicalEditi } \\
\text { ng } \backslash\end{array}} &{2} &{ } \\
{\hline \text { Unit1.pas }} &{\text { C:ISaphire 8\Debug \source \DelphiSource } \backslash} &{1} &{ } \\
{\hline \text { dummyone.pas (Unit2) }} &{\text { C: \Saphire 8\Debug \source \DelphiSource } \backslash} &{0} &{ } \\
{\hline \text { dummy3.pas (Unit3) }} &{\text { C: \Saphire 8\Debug \source \DelphiSource } \backslash} &{0} &{ } \\
{\hline \text { UnorderedBDD.pas }} &{\begin{array}{l}\text { C: } \backslash \text { Saphire } \\
8 \backslash \text { Debug } \backslash \text { source } \backslash \text { DelphiSource } \backslash B d d I t e m s \backslash\end{array}} &{3} &{ } \\
{\hline \text { UpdLib.pas }} &{\text { C:|Saphire 8\Debug|source } \backslash} &{4} &{ } \\
{\hline \text { UpgradeCats.pas }} &{\text { C: \Saphire } 8 \backslash \text { Debug } \backslash \text { source } \backslash \text { DelphiSource } \backslash} &{4} &{ } \\
{\hline \text { UpgradeETLogic.pas }} &{\text { C: \Saphire 8\Debug \source \DelphiSource } \backslash} &{13} &{ } \\
{\hline \text { Variates.pas }} &{\text { C: \Saphire 8\Debug \source \DelphiSource } \backslash} &{3} &{ } \\
{\hline \text { VirtualNodeEditor.pas }} &{\text { C: \Saphire 8\Debug \source \DelphiSource } \backslash} &{2} &{ } \\
{\hline \text { VRPT.pas }} &{\text { C:|Saphire 8\Debug|source } \backslash} &{0} &{ } \\
{\hline \text { VRptLib.pas }} &{\text { C:ISaphire 8\Debug|source } \backslash} &{13} &{ } \\
{\hline \text { VSTreeStateObj.pas }} &{\text { C: \Saphire } 8 \backslash \text { Debug } \backslash \text { source } \backslash \text { DelphiSource } \backslash} &{10} &{ } \\
{\hline \text { VTEditors.pas }} &{\text { C: \Saphire 8\Debug \source \DelphiSource } \backslash} &{2} &{ } \\
{\hline \text { WebSiteLib.pas }} &{\text { C:ISaphire 8\Debug \source \DelphiSource } \backslash} &{4} &{ } \\
{\hline \text { WEditLib.pas }} &{\text { C:\Saphire } 8 \backslash \text { Debug } \backslash \text { source } \backslash} &{11} &{ } \\
{\hline \text { WinAux.pas }} &{\text { C:ISaphire 8\Debug|source\} } &{3} &{ } \\
{\hline \text { WinDevice.pas }} &{\text { C:|Saphire 8\Debug|source\} } &{4} &{ } \\
{\hline \text { Window.pas }} &{\text { C:ISaphire 8\Debug|source\} } &{2} &{ } \\
{\hline \text { WinDriver.pas }} &{\text { C:|Saphire 8\Debug \source\} } &{5} &{ } \\
{\hline \text { WinExec.pas }} &{\text { C:|Saphire 8\Debug|source\} } &{6} &{ } \\
{\hline \text { WinHelp.pas }} &{\text { C:|Saphire 8\Debug|source } \backslash} &{9} &{ } \\
{\hline \text { WinKey.pas }} &{\text { C:|Saphire 8\Debug|source\} } &{31} &{ } \\
{\hline \text { WinPrint.pas }} &{\text { C:|Saphire 8\Debug|source\} } &{5} &{ } \\
{\hline \text { WinRebuild.pas }} &{\text { C:|Saphire 8\Debug|source\} } &{9} &{ } \\
{\hline \text { WinReports.pas }} &{\text { C:ISaphire 8\Debug|source } \backslash} &{9} &{ } \\
{\hline \text { WinRpUtil.pas }} &{\text { C:|Saphire 8\Debug|source\} } &{9} &{ } \\
\hline\end{array}$
\end{tabular}

Page 28 


\begin{tabular}{|c|c|c|c|}
\hline \multirow[t]{2}{*}{ File Name } & \multirow[t]{2}{*}{ File Path } & \multicolumn{2}{|c|}{$\begin{array}{l}\text { Cyclomatic } \\
\text { Complexity }\end{array}$} \\
\hline & & v8.06.01 & v8.07.17 \\
\hline WinSpec.pas & C:|Saphire $8 \backslash$ Debug $\backslash$ source $\backslash$ & 0 & \\
\hline WinText.pas & C:|Saphire 8\Debuglsourcel & 6 & \\
\hline WinTools.pas & C:|Saphire $8 \backslash$ Debug $\backslash$ source $\backslash$ & 3 & \\
\hline WorkspaceUtils.pas & C:|Saphire $8 \backslash$ Debug $\backslash$ source $\backslash$ DelphiSource $\backslash$ & 5 & \\
\hline WorkspaceUtils.pas & C:|Saphire $8 \backslash$ Debug $\backslash$ source $\backslash$ DelphiSource $\backslash$ & 5 & \\
\hline WriteHTML.pas & C: $\backslash$ Saphire $8 \backslash$ Debug $\backslash$ source $\backslash$ DelphiSource $\backslash$ & 2 & \\
\hline XmlDataGenerator.pas & C:|Saphire 8\Debug|sourcel & 2 & \\
\hline XmlSchemaGenerator.pas & C:|Saphire 8\Debug \source $\backslash$ & 2 & \\
\hline
\end{tabular}

Figure 3-1 provides the SAPHIRE Version 8 Project Analysis using the Audits and Checks category options provided in Figure 3-2 and Figure 3-3. The analysis parses all source files into an internal tree to ensure that all symbols are available to be cross referenced and validated. The analysis results provide information on the following:

- Parsing errors

- Delphi directive names used as identifiers

- Delphi keywords used as identifiers

- Identifiers hiding others of the same name at a higher scope

- "if" statements followed by an empty statement

- Type assignment incompatibilities

- Undefined function return values

- Uninitialized variables

- Unreferenced global identifiers

- Unreferenced local identifiers

- Unused assigned values

The following provide the definitions for the messages as a result of the analysis:

A1001 Audit result message - The <item $>$ is declared as deprecated.

A1002 Audit result message - The identifier $<\mathrm{id}>$ is also a Delphi directive.

A1003 Audit result message - The $<$ item $>$ is declared as experimental.

A1004 Audit result message - The $<$ item $>$ hides the $<$ item $>$ of the same name declared on line $<\mathrm{n}>$ of $<$ filename $>$.

A1005 Audit result message - The identifier $<$ id $>$ is also a Delphi Keyword. 
A1006 Audit result message - The $<$ item $>$ is declared as library dependent.

A1007 Audit result message - The $<$ item $>$ is declared as platform dependent.

C1001 Check result message - The array index expression can be outside the bounds of the array.

C1002 Check result message - The destination of the assignment is a constant so should not be modified.

$\mathbf{C 1 0 0 3}$ Check result message - The "if" statement is followed by an empty statement.

C1004 Check result message - The constant expression is outside the range of valid values.

C1005 Check result message - The type $<$ type $>$ and $<$ type $>$ are not compatible.

C1006 Check result message - The return value of $<$ function $>$ is undefined in one or more code paths through its implementation.

C1007 Check result message - The <item $>$ has not been assigned a value in one or more code paths.

$\mathbf{C 1 0 0 8}$ Check result message - The source code at or before $<$ item $>$ is unreachable.

C1009 Check result message - The global <item $>$ is declared but is never referenced.

C1010 Check result message - The local $<$ item $>$ is declared but is never referenced.

C1011 Check result message - The value assigned to $<\mathrm{id}>$ is never used.

To access the file contents shown in Figure 7-1, refer to file "SAPHIRE8-Analysis-100405.pdf" included on the $\mathrm{CD}$ as an addendum to this report. To access the file:

1. Open Internet Explorer.

2. Select "Open" from the "File" menu.

3. The "Open" window will be displayed.

4. Select the "Browse..." button from the "Open" window.

5. The "Windows Internet Explorer" window will be displayed.

6. Navigate to the "SAPHIRE8-Analysis-100405.pdf" file located on the CD.

7. Select the "SAPHIRE8-Analysis-100405.pdf" file on the CD.

8. Select the "Open" button from the "Windows Internet Explorer" window.

9. The "Windows Internet Explorer" window will close.

10. Select the "OK" button from the "Open" window. 
File Edit view Options Help.

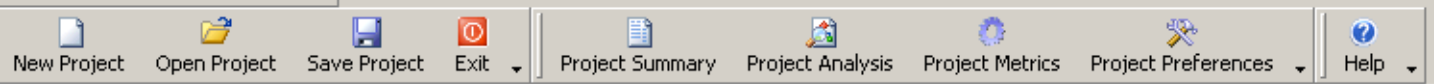

CodeHealer analysis for project SAPHIRE 8

I Analyse

Program entities

क. Source modules (683)

+ 8 <Library>

+6 <Library>

$+\&\langle$ Library>

+8 LLibrary $\rangle$

$+\$\langle$ Library>

3 〈Package $\rangle$

+3 <Program $\rangle$

+6 〈Program〉

+9 〈Program>

+6 <Unit> [Imp]

+2 <Unit> [Imp]

+3 <Unit> [Imp]

+t 3 <Unit> [lmp]

+2 <Unit> [lmp]

+4 〈Unit> [Imp]

+3 〈Unit> [Imp]

+ 3 〈Unit> [Imp] Name

Name

CCFRDLL

HRADLL

PlugCCFStag

PlugDFM

SageST

SageSTPackage

SageSTSchema0bjects

SAPHIRE

SAPHIRE_Project

AboutUnit

AdiListPanel

AnalysisPointETObject

AnalysisPointFTCSPathObject

AnalysisPointFTObject

AnalysisPoint0bject

AnalyzeGeneral

AnalyzeSDP

Analysis results

Check description

(9) Check failures (13204) * Parsing errors *

(t) Array index expressions out of bounds [150)

+ Delphi directive names used as identifiers [65]

[+. Delphi keywords used as identifiers [2]

(†) Identifiers hiding others of the same name at a higher scope [3249]

(1) If statements followed by an empty statement [87]

(†) Type assignment incompatibilities [2]

(t) Undefined function return values [106]

†. Uninitialised variables [823]

+ Unreferenced global identifiers [7929]

†+ Unreferenced local identifiers [374]

†. Unused assigned values [417]

(8) Parsing errors (7)

圈 Project Summary Project Analysis Project Metrics

Figure 3-1 Source Code Analysis for CodeHealer SAPHIRE 8 v8.06.01 


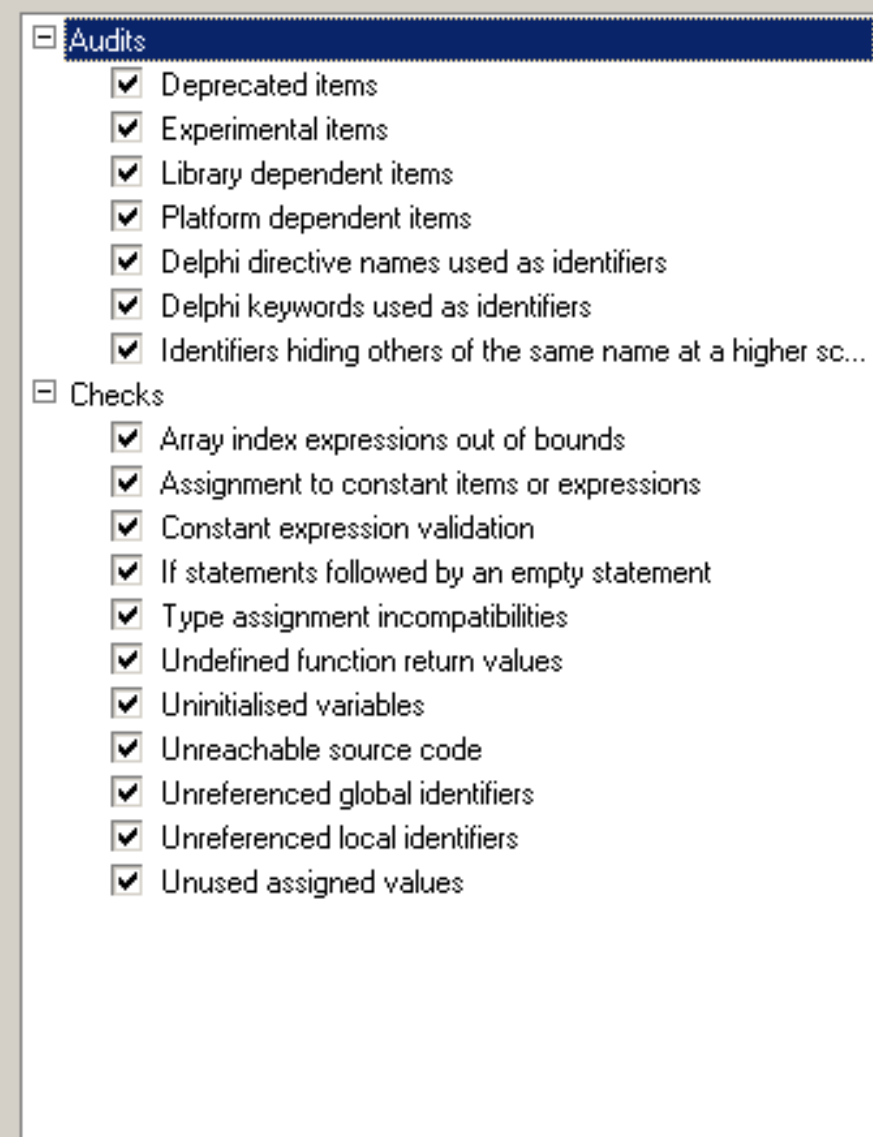

\section{Audits}

The Audits category consists of quality control issues such as unused or unreachable code, use of Delphi directive names and keywords as identifiers, identifiers hiding others of the same name at a higher scope, and more.

$\checkmark$ Delphi directive names used as identifiers

$\checkmark$ Delphi keywords used as identifiers

$\checkmark$ Identifiers hiding others of the same name at a higher sc...

$\checkmark$ Array index expressions out of bound

$\checkmark$ Assignment to constant items or expressions

$\checkmark$ Constant expression validation

If statements followed by an empty statement

$\checkmark$ Type assignment incompatibilities

$\checkmark$ Undefined function return values

$\checkmark$ Uninitialised variables

$\checkmark$ Unreachable source code

$\checkmark$ Unreferenced global identifiers

$\checkmark$ Unreferenced local identifier

$\checkmark$ Unused assigned values

Reset to default $\quad$ Cancel

Figure 3-2 Audits Category for CodeHealer Project SAPHIRE 8 Analysis 


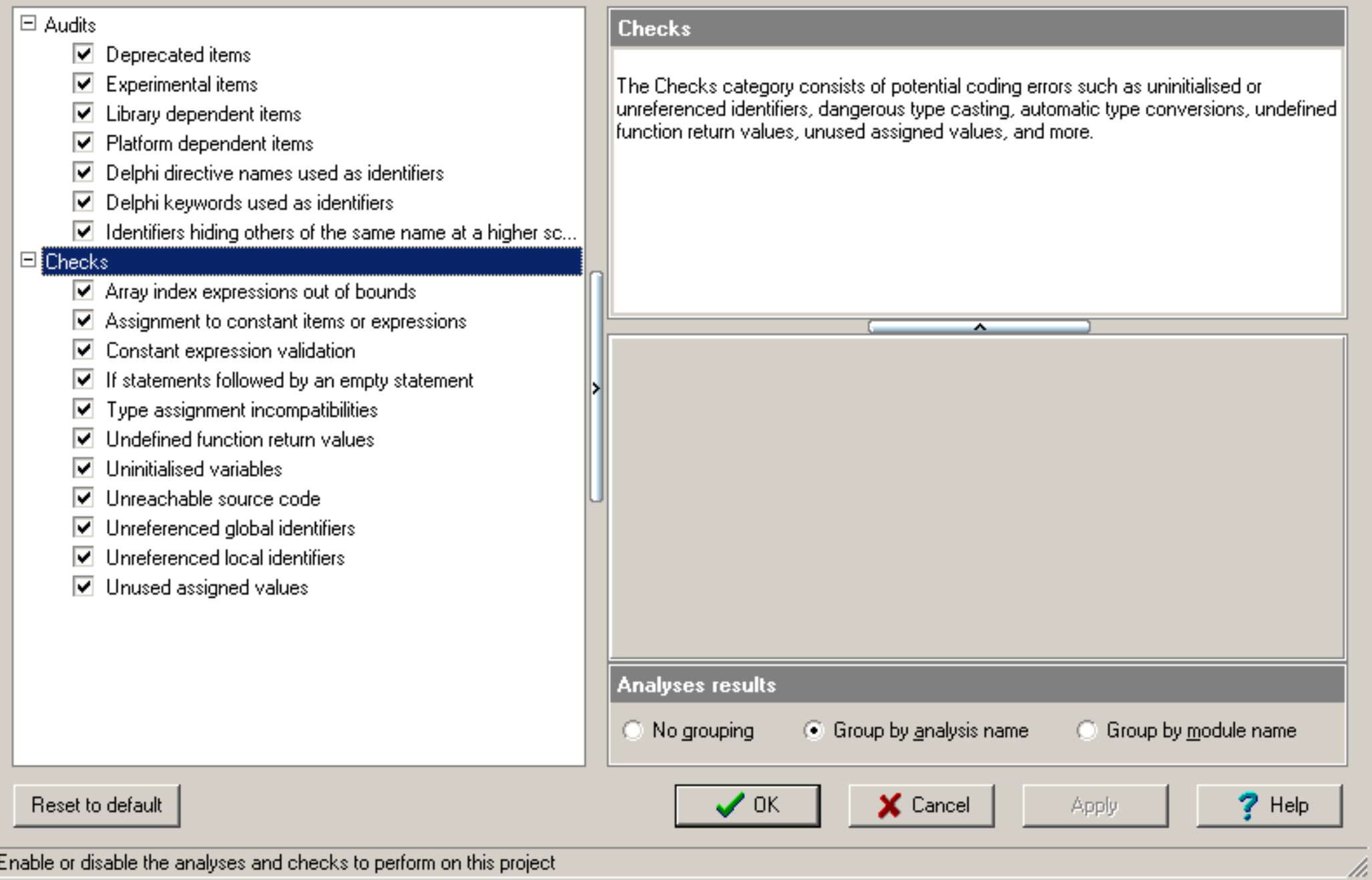

Figure 3-3 Checks Category Settings for CodeHealer Project SAPHIRE 8 Analysis 


\subsection{Coverage Validator}

Coverage Validator by Software Verification, Copyright Object Media Limited (C 2002-2006. Coverage Validator is an automatic source code coverage analyzer for Windows NT® 4.0 and above, running on the Intel i386 (and compatible) family of processors. Coverage Validator automatically detects which lines of your program have been executed, provides visit counts for each line and calculates visit percentages for files that are visited. Coverage Validator places a very low overhead on the performance of your application and does not require the target program to be recompiled or relinked. Coverage Validator can also be used for unit testing and as part of a regression testing strategy used by Quality Assurance teams.

\subsubsection{Coverage Validator Results}

Version 8.06.1 was used to run an SDP, ECA and GA in the IV\&V effort. We will run the same analyses types for v8.07.17 and present both of the results for the metrics report.

Figure 8-1, Figure 8-2 and Figure 8-3 provides the coverage summary for the SAPHIRE version 8 source code using the source code coverage analyzer Coverage Validator (C) Version 4.10. The coverage summary for a Significance Determination Process analysis (Figure 8-1), Events and Condition Assessment analysis (Figure 8-2) and a General analysis (Figure 8-3) have been provided.

The figures show various coverage statistics with percentages where appropriate. No unit testing has been performed. The statistics displayed are:

- Number of files

- Number of visited files

- Number of unvisited files

- Number of $100 \%$ visited files

- Number of functions

- Number of visited functions

- Number of unvisited functions

- Number of $100 \%$ visited functions

- Number of branches

- Number of visited branches

- Number of unvisited branches

- Number of functions containing branches

- Number of $100 \%$ branch functions 
- Number of lines

- Number of visited lines

- Number of unvisited lines

- Number of lines that could not be hooked

- Number of visits to lines

- Number of unit tests

- Number of successful unit tests

- Number of unsuccessful unit tests

- Number of unit test failures

- Number of unit test errors

\subsection{Memory Validator}

Memory Validator by Software Verification, Copyright Object Media Limited C 2002-2006.

Memory Validator is an advanced memory and resource leak detector for Windows NT® 4.0 and above, running on the Intel i386 (and compatible) family of processors. Memory Validator detects memory leaks, handle leaks, memory corruptions, uninitialized memory, buffer overruns and buffer under runs. Memory Validator is fast and does not require the target program to be recompiled or relinked. Memory Validator can also be used to detect performance problems related to memory and resource allocation and deallocation, and performance problems related to fragmented memory heaps. Memory Validator tracks the $\mathrm{C}$ runtime heaps, Win32 heaps, GlobalAlloc and LocalAlloc heaps, VirtualAlloc heaps, Win32 resource handles and COM object creation and destruction.

\subsubsection{Memory Validator Results}

Figure 3-4 provides the results for memory leak and handle leak detection for the SAPHIRE version 8 source code v8.06.01 using the memory and resource leak detector Memory Validator (C) Version 4.86. The option "Check pointers and handles on DWORD boundaries. Fastest option. This will find less pointers and handles, but also find less FALSE positives" was selected for the results provided. 


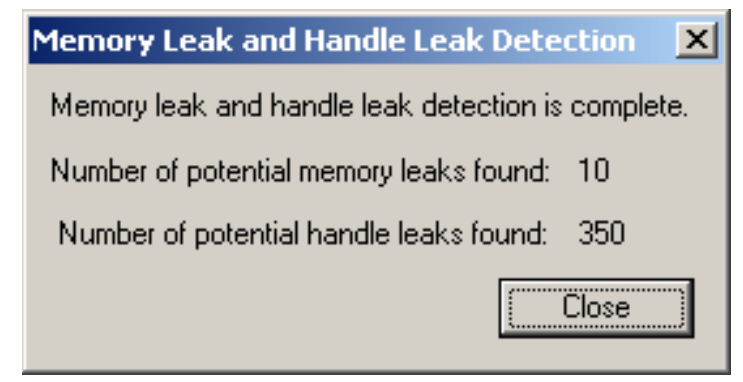

Figure 3-4 Memory Validator Memory Leak and Handle Leak Detection v8.06.01

Figure 3-5 provides the results for memory leak and handle leak detection for the SAPHIRE version 8 source code v8.07.17 using the memory and resource leak detector Memory Validator (C) Version 4.86.

Figure 3-5 Memory Validator Memory Leak and Handle Leak Detection v8.07.17

\subsection{Performance Validator}

Performance Validator by Software Verification, Copyright Object Media Limited C 2002-2006. Performance Validator is an automatic performance analyzer for Windows NT® 4.0 and above, running on the Intel i386 (and compatible) family of processors. Performance Validator automatically calculates performance data for your application as it executes. Performance Validator places a very low overhead on the performance of your application and does not require the target program to be recompiled or relinked. Performance Validator can also be used for unit testing and as part of a regression testing strategy used by Quality Assurance teams.

\subsubsection{Performance Validator Results}

Figure 3-6 through Figure 3-11 provides partial results for the performance statistics for the SAPHIRE version 8 source code using the performance analyzer Performance Validator (C) Version 2.63. The performance statistics for a Significance Determination Process analysis (Figure 3-6 and Figure 3-7), Events and Condition Assessment analysis (Figure 3-8 and Figure 3-9) and a General analysis (Figure 3-10 and Figure 3-11) have been provided.

The figures show various performance statistics with percentages where appropriate. The statistics displayed are:

Number of Children - The number of child functions of a function.

Address - The function address.

Class - The class name.

Method / Function Name - The method/function name.

File Name - The file name. 
Module Name - The module name.

Call Count - The number of times the function is called.

Call Count $\%$ - The number of times the function is called.

Function Time - The time a function takes to execute.

Function Time $\%$ - The time a function takes to execute.

Total Time - The time a function and its child functions take to execute.

Total Time $\%$ - The time a function and its child functions take to execute.

Average Time - The average time a function takes to execute.

Average Time $\%$ - The average time a function takes to execute.

Child Time - The time a function's child functions take to execute.

Child Time $\%$ - The time a function's child functions take to execute.

Longest Time - The longest time a function and its child functions take to execute.

Longest Time $\%$ - The longest time a function and its child functions take to execute.

Shortest Time - The shortest time a function and its child functions take to execute.

Shortest Time $\%$ - The shortest time a function and its child functions take to execute.

This report identifies functions and methods that are taking the most time, or which are visited more than other functions. 


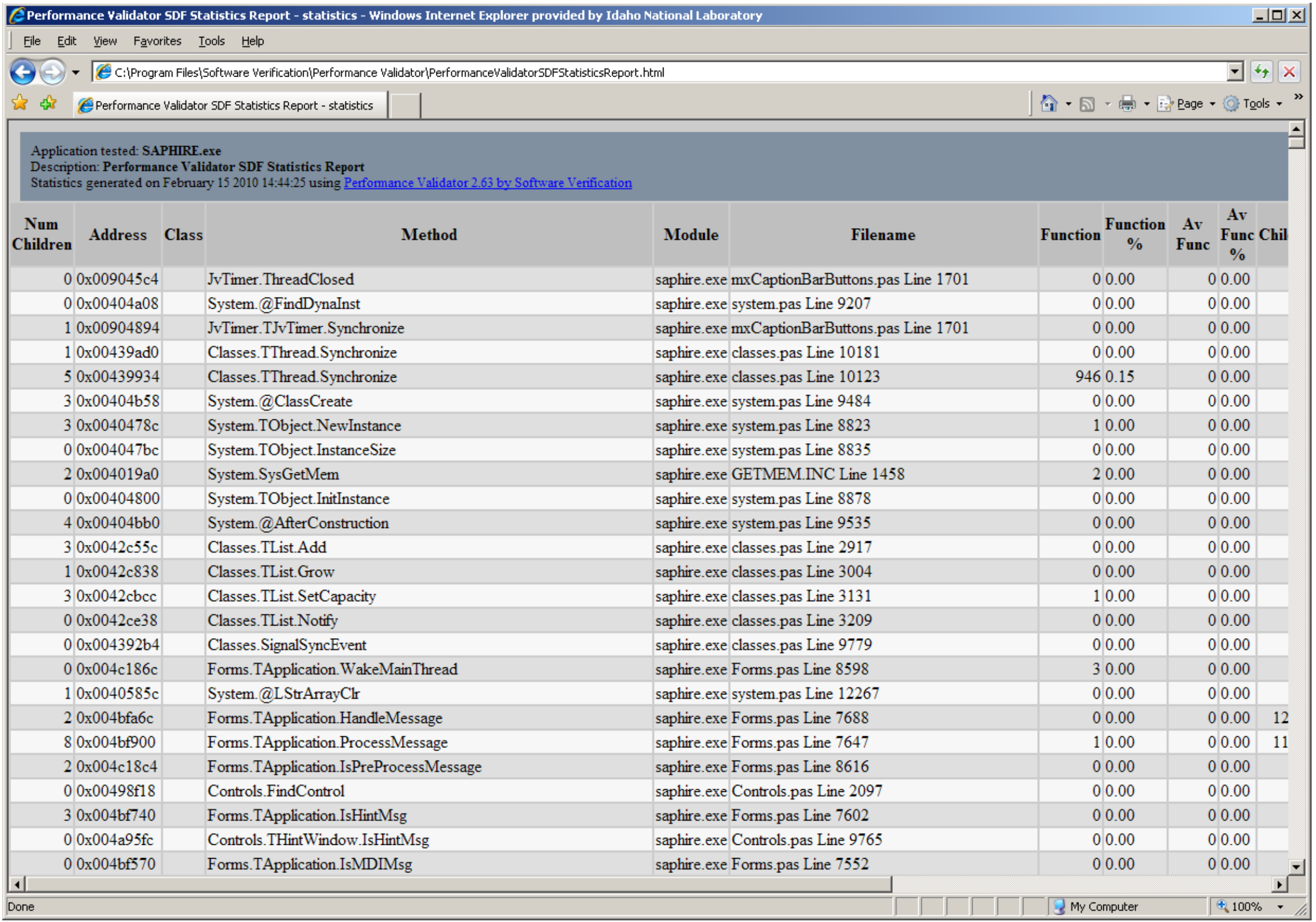

Figure 3-6 SAPHIRE 8 v8.06.01 Performance Validator SDP Statistics Report - Left 


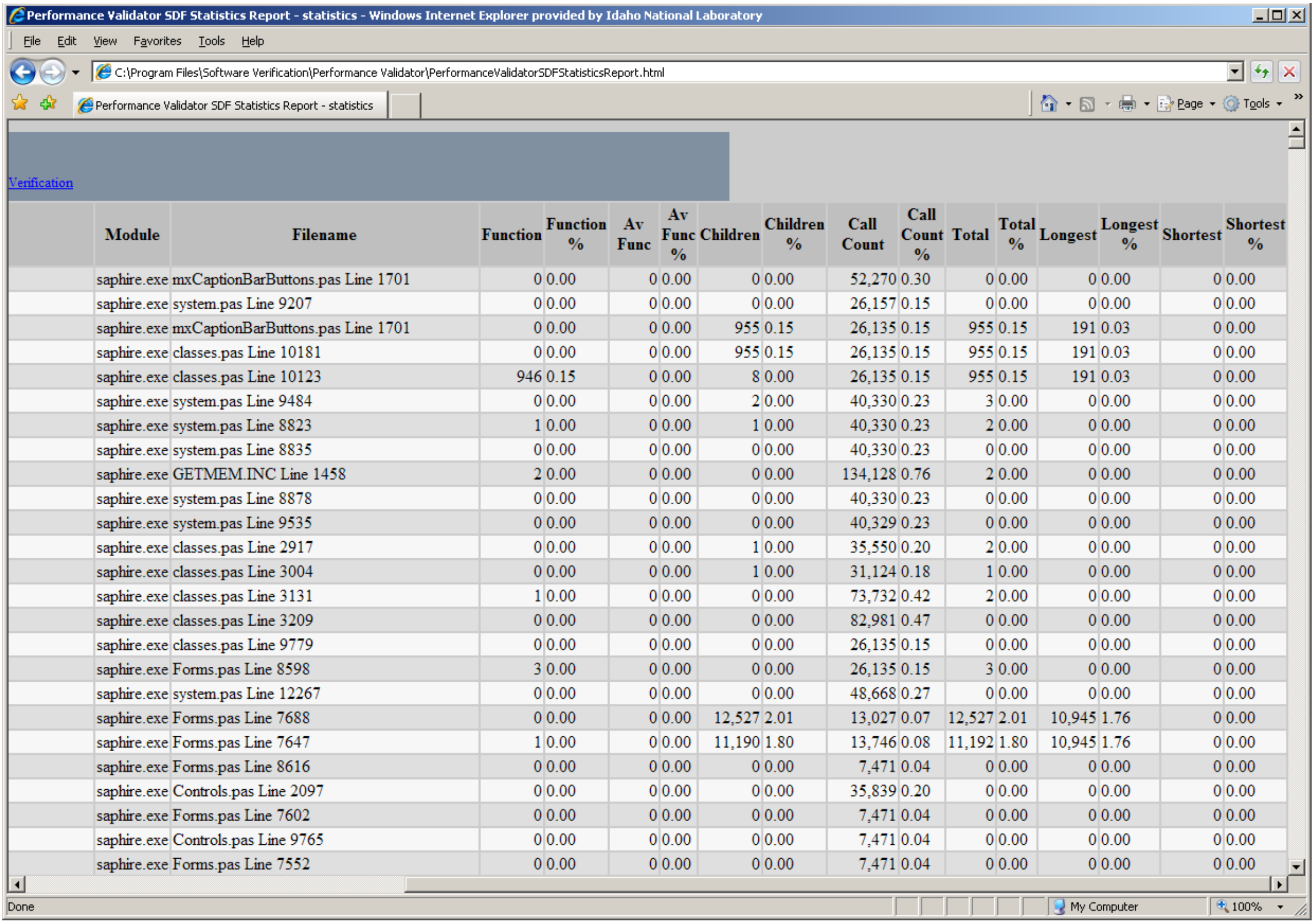

Figure 3-7 SAPHIRE 8 v8.06.01 Performance Validator SDP Statistics Report - Right 


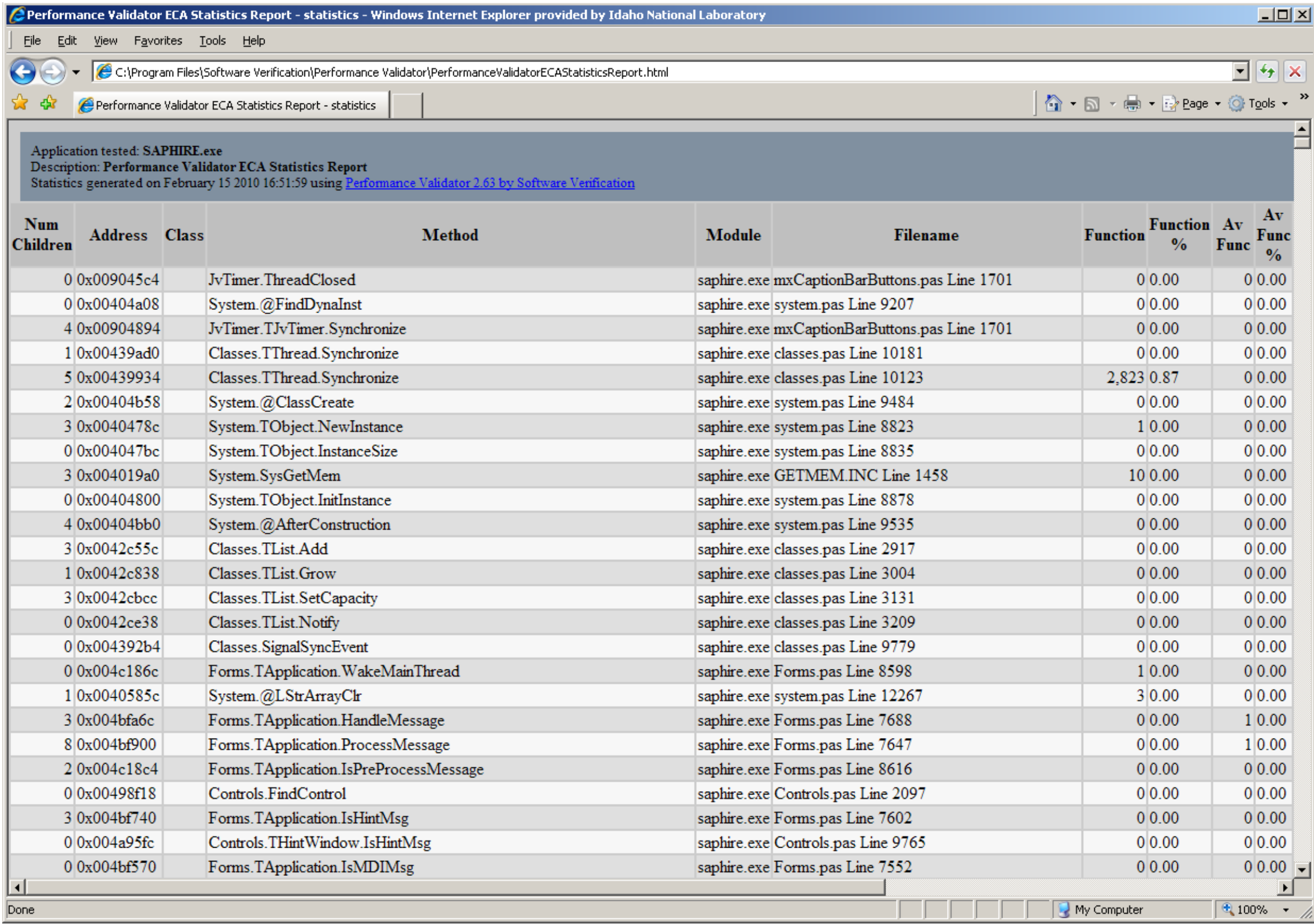

Figure 3-8 SAPHIRE 9 v8.06.01 Performance Validator ECA Statistics Report - Left 


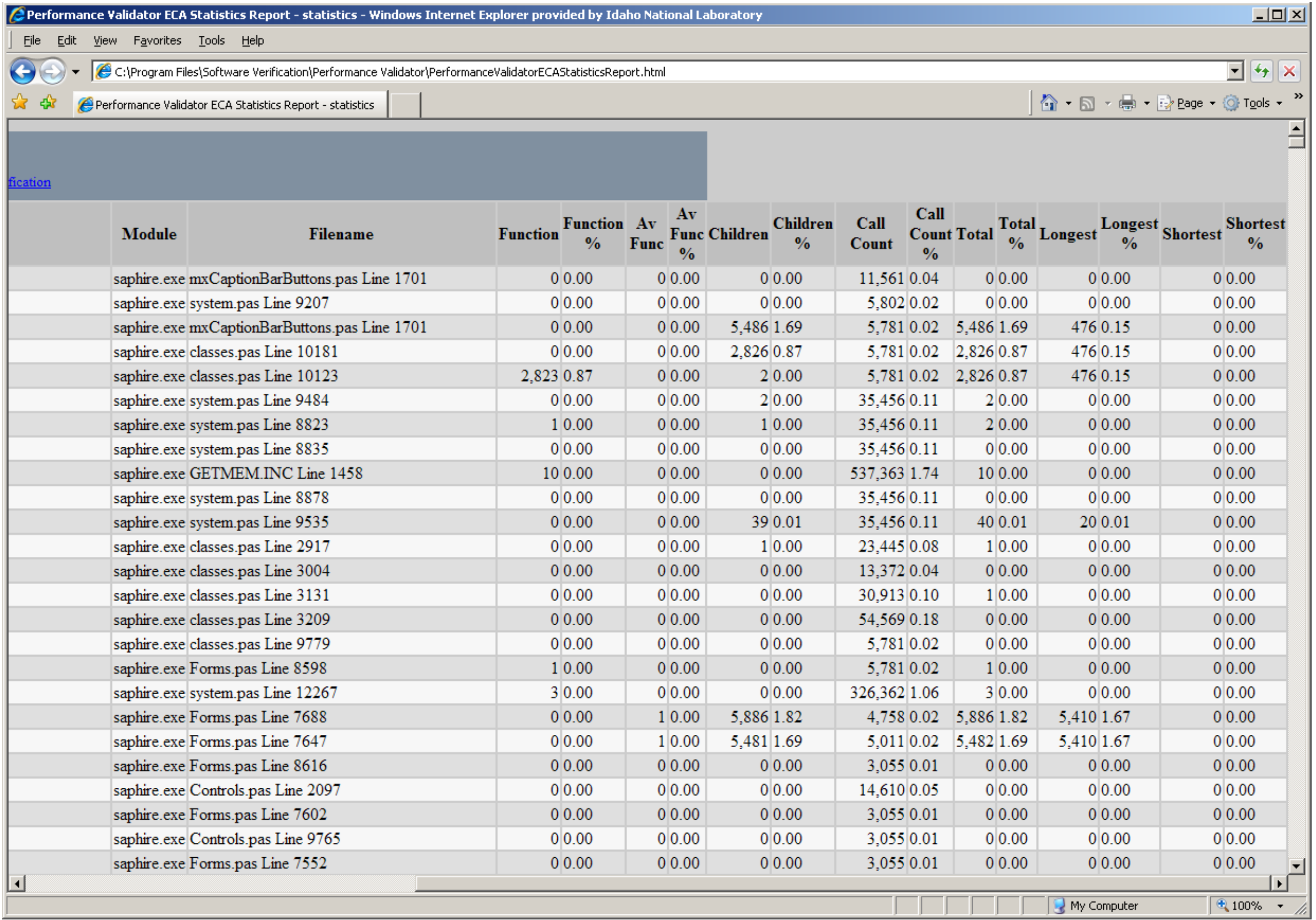

\section{Figure 3-9 SAPHIRE 8 v8.06.01 Performance Validator ECA Statistics Report - Right}




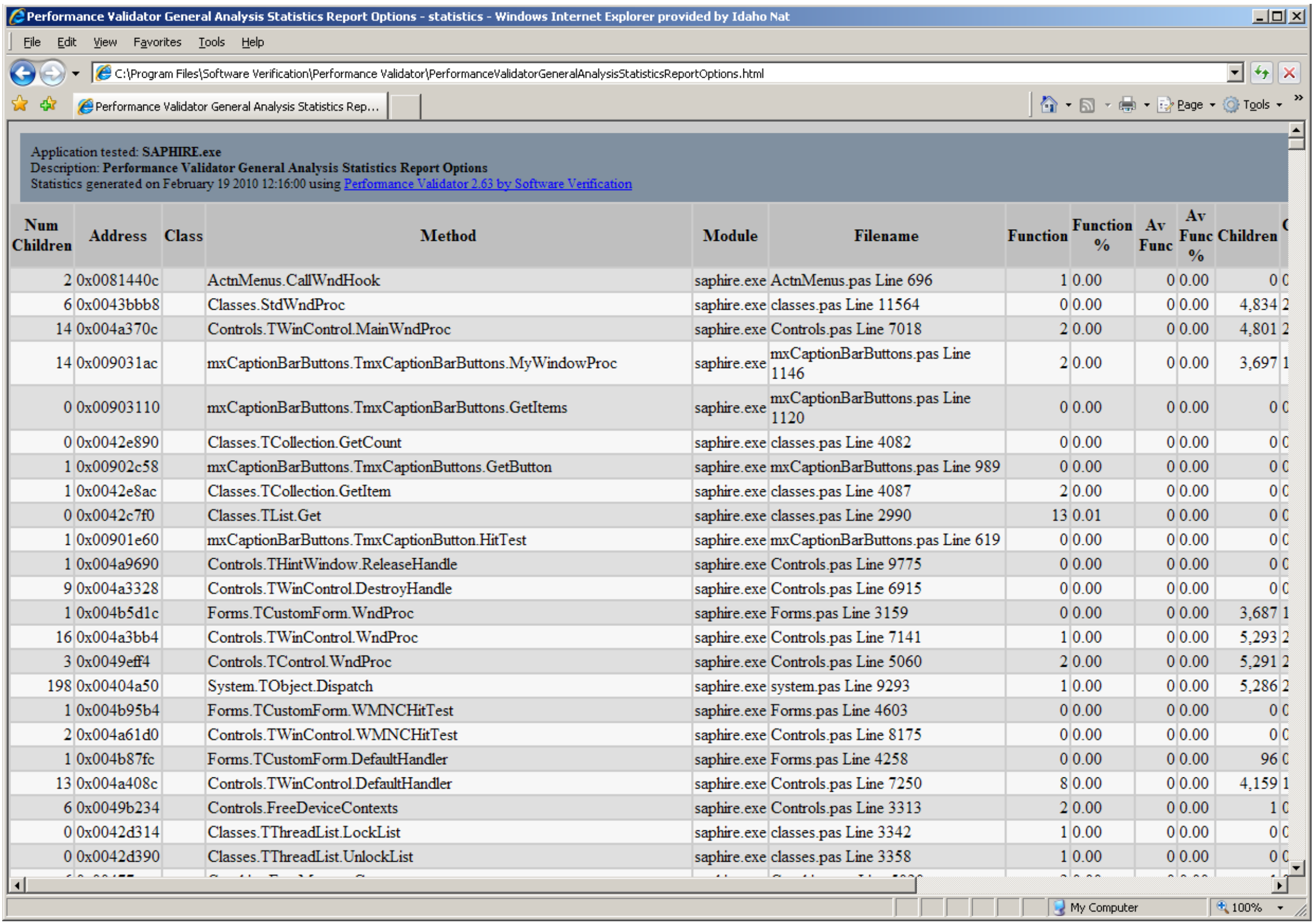

Figure 3-10 SAPHIRE 8 v8.06.01 Performance Validator General Analysis Statistics Report - Left 


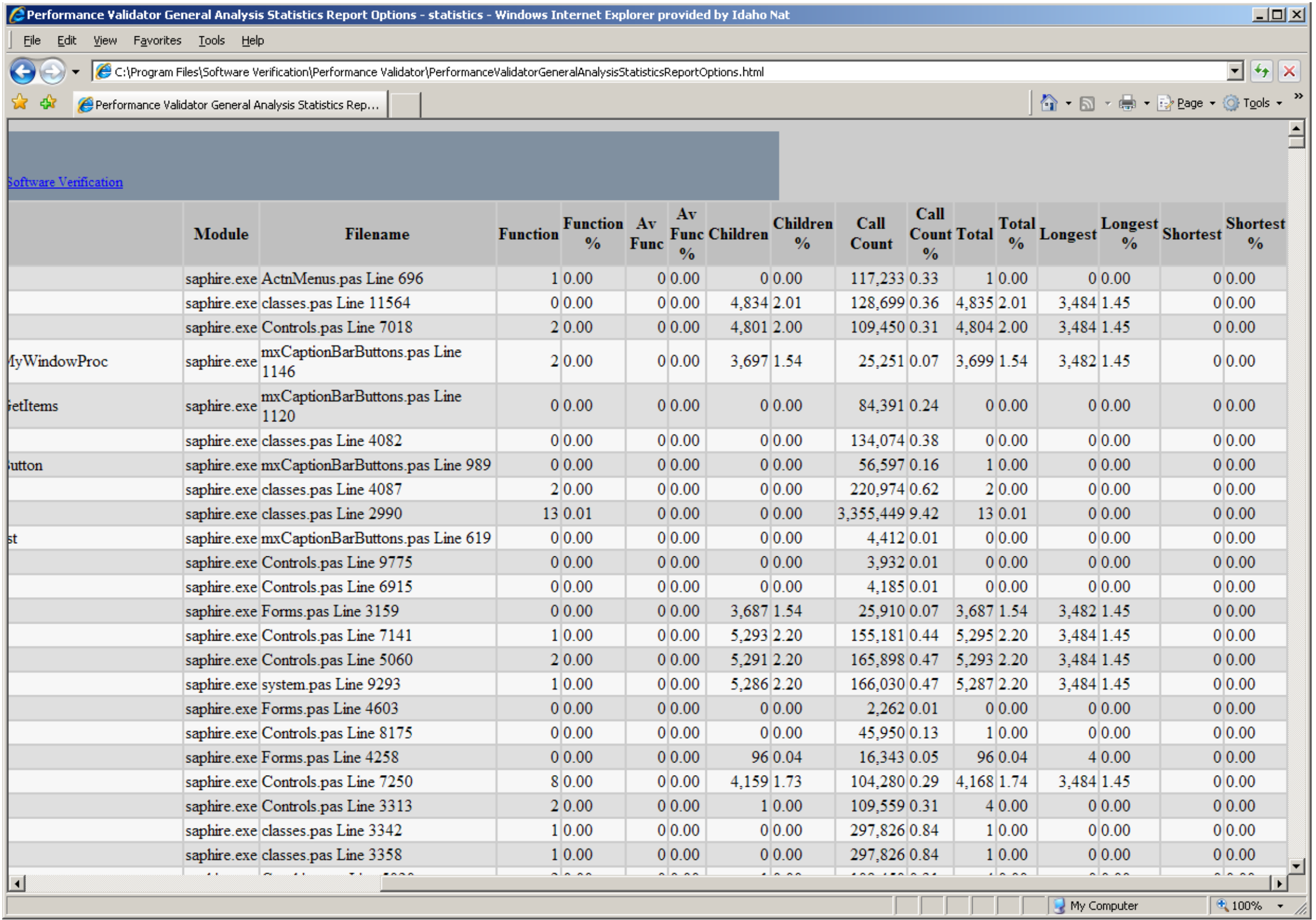

\section{Figure 3-11 SAPHIRE 8 v8.06.01 Performance Validator General Analysis Statistics Report - Right}




\subsection{Thread Validator}

Thread Validator by Software Verification, Copyright Object Media Limited (C) 2003-2006. Thread Validator is an automatic deadlock detector for Windows NT® 4.0 and above, running on the Intel i386 (and compatible) family of processors. Thread Validator detects deadlocks, potential deadlocks, bad lock strategies, and performs deadlock postmortem analysis. Thread Validator places a very low overhead on the performance of your application and does not require the target program to be recompiled or relinked.

\subsubsection{Thread Validator Results}

Figure 3-13, Figure 3-14 and Figure 3-15 provide the deadlocks, potential deadlocks, bad lock strategies, and deadlock postmortem analysis for the SAPHIRE version 8 source code using the deadlock detector Thread Validator Version 1.52. The partial results of deadlocks, potential deadlocks, bad lock strategies, and deadlock postmortem analysis for a Significance Determination Process analysis (Figure 3-13), Events and Condition Assessment analysis (Figure 3-14) and a General analysis (Figure 3-15) have been provided. Figure 8-16 provides the color code definitions for the memory and resource allocations that have not been de-allocated and any information about damaged memory presented in Figure 3-13 through Figure 3-15. 


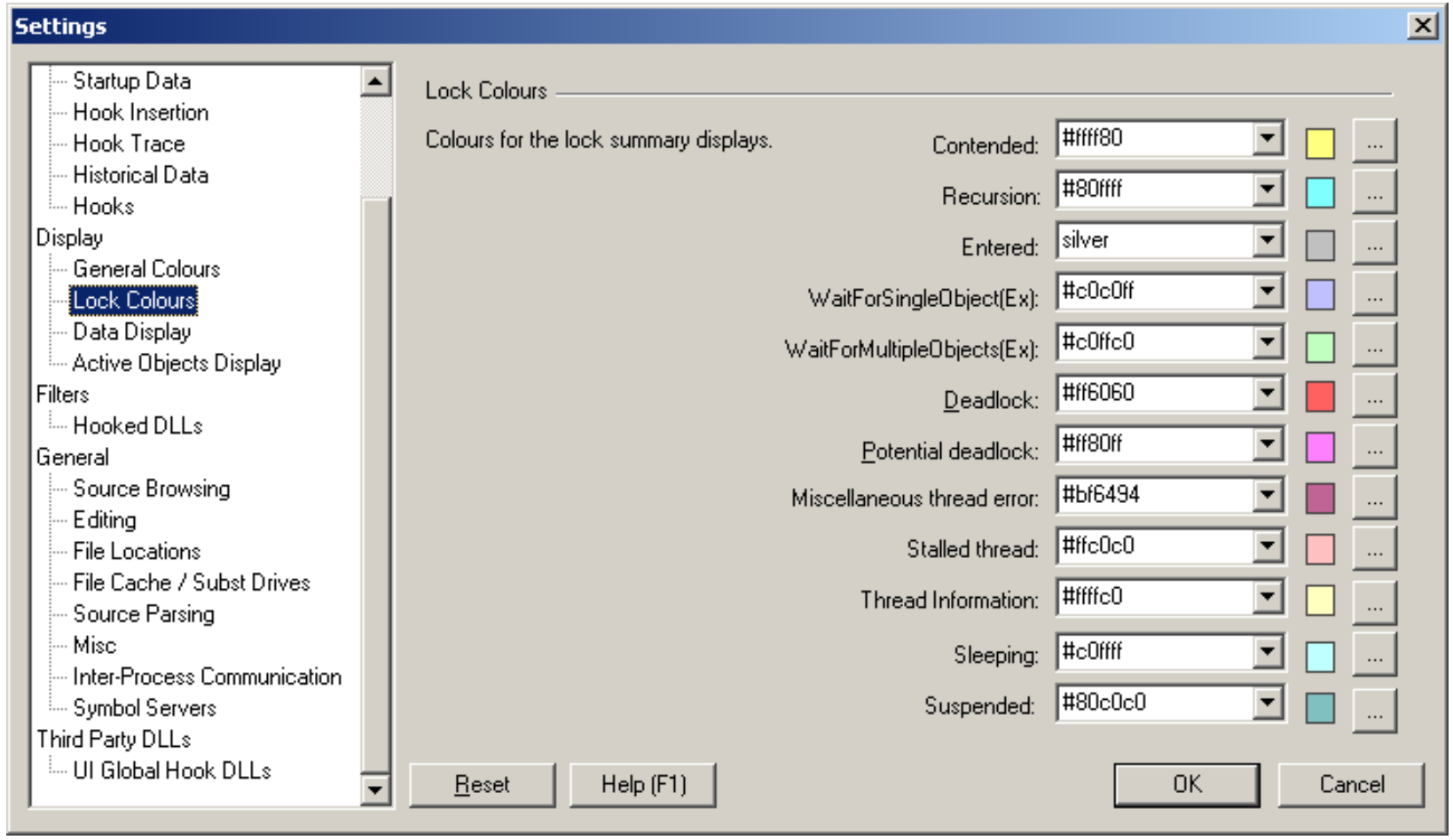

Figure 3-12 Thread Validator Lock Colors Definitions

Figure 3-13 through Figure 3-15 provide the following information about all memory and resource allocations that have not been deallocated and any information about damaged memory:

The first column provides the Critical Section Address.

The second column provides the Entered Count.

The third column provides the Recursion Count, the number of times the critical section has been re-entered while locked by the current thread.

The fourth column provides the Contended Count, the number of times that a thread has tried to acquire the critical section but has had to wait because another thread already owns the critical section.

The fifth column provides the Number of Waiting Processor Cycles.

The sixth column provides the Thread Id.

The seventh column provides the Number of Waiting Threads.

The eighth column provides the Location Where the Object Was Allocated, Locked or Waiting. 


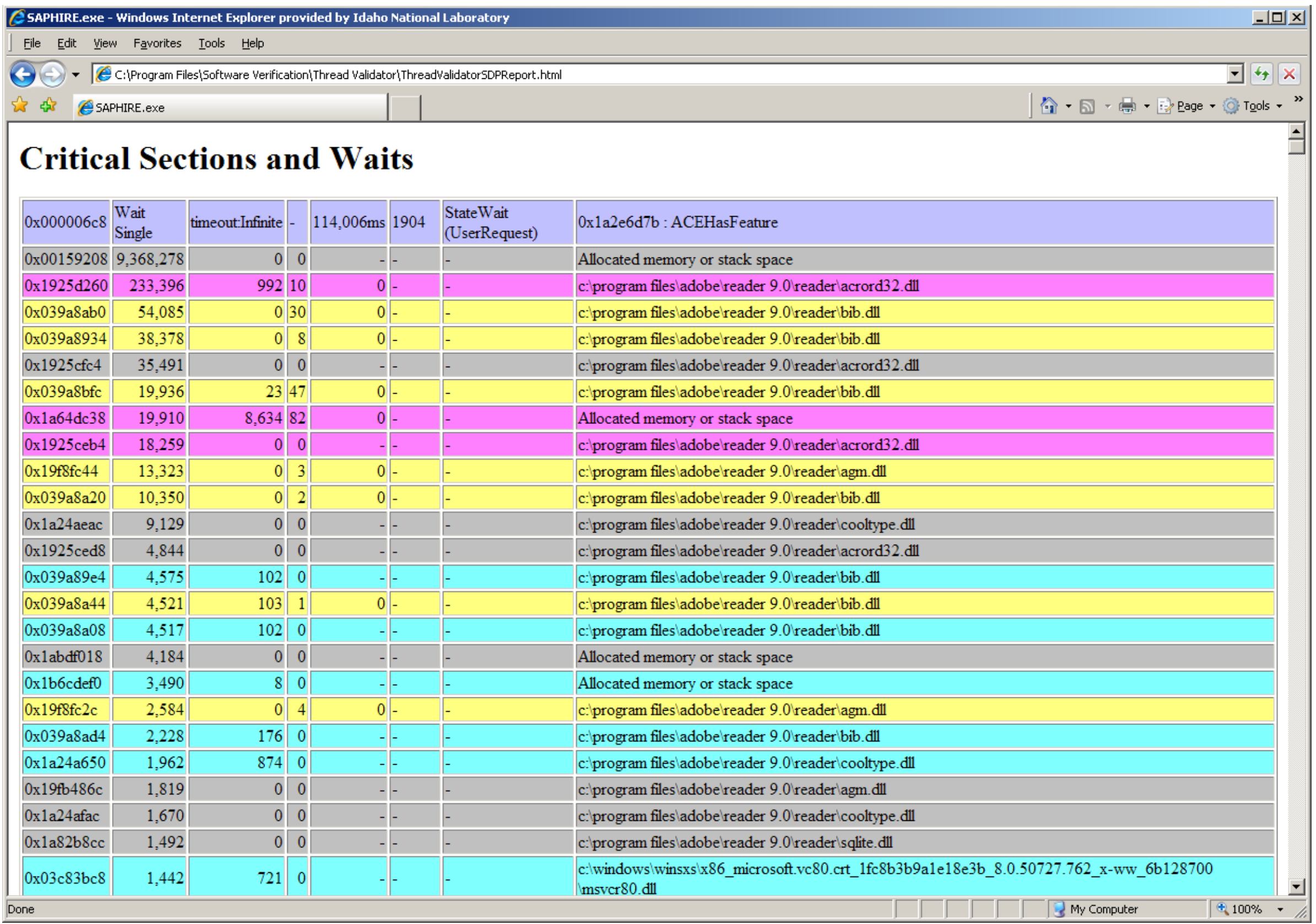

Figure 3-13 SAPHIRE 8 v8.06.01Thread Validator SDP Critical Sections and Waits Report 


\section{Critical Sections and Waits}

\begin{tabular}{|c|c|c|c|c|c|c|c|}
\hline $0 x 00000698$ & Wait Single & timeout:Infinite & - & $47,026,369 \mathrm{~ms}$ & 6140 & $\begin{array}{l}\text { StateWait } \\
\text { (UserRequest) }\end{array}$ & 0x19ae6d7b : ACEHasFeature \\
\hline 0x00159208 & $46,480,854$ & 0 & 0 & & - & - & Allocated memory or stack space \\
\hline $0 \mathrm{x} 18 \mathrm{a} 5 \mathrm{~d} 260$ & $31,134,745$ & 1,123 & 5 & 0 & - & - & c: program files ladobelreader 9.0 reader lacrord32. dll \\
\hline $0 \mathrm{x} 18 \mathrm{a} 5 \mathrm{cfc} 4$ & $9,995,464$ & 0 & 0 & - & - & - & c: program files ladobelreader 9.0 reader lacrord32. dll \\
\hline $0 \mathrm{x} 18 \mathrm{a} 5 \mathrm{ceb} 4$ & $4,849,360$ & 0 & 0 & & - & - & c: program files ladobelreader 9.0 reader lacrord32. dll \\
\hline $0 x 19 e 4 d c 38$ & $3,455,724$ & $1,477,598$ & 2 & 0 & - & - & Allocated memory or stack space \\
\hline 0x1ab3f018 & $3,005,905$ & 0 & 0 & & - & - & Allocated memory or stack space \\
\hline $0 \mathrm{x} 01 \mathrm{e} 08 \mathrm{ab} 0$ & 54,083 & 0 & 5 & 0 & - & - & c: program files ladobelreader 9.0 reader bib.dll \\
\hline $0 \mathrm{x} 01 \mathrm{e} 08934$ & 31,039 & 0 & 1 & 8 & - & - & c: program files ladobelreader 9.0 reader bib.dll \\
\hline $0 \mathrm{x} 01 \mathrm{e} 08 \mathrm{bfc}$ & 20,390 & 24 & 0 & & - & - & c: program files ladobelreader 9.0 reader bib.dll \\
\hline $0 \mathrm{x} 1978 \mathrm{fc} 44$ & 19,257 & 0 & 2 & 0 & - & - & c: program files ladobelreader 9.0 readerlagm.dll \\
\hline $0 \mathrm{x} 01 \mathrm{e} 08 \mathrm{a} 20$ & 10,385 & 0 & 0 & - & - & - & c: program files ladobelreader 9.0 reader bib.dll \\
\hline 0x19a4aeac & 6,911 & 0 & 0 & & - & - & c: program files $\mid$ adobelreader 9.0 reader cooltype.dll \\
\hline $0 \times 18 a 5 c e d 8$ & 4,844 & 0 & 0 & & - & - & c: program files ladobelreader 9.0 readerlacrord32. dll \\
\hline $0 \mathrm{x} 1978 \mathrm{fc} 2 \mathrm{c}$ & 3,518 & 0 & 1 & 0 & - & - & c: program files $\backslash$ adobelreader 9.0 reader lagm.dll \\
\hline $0 \mathrm{x} 01 \mathrm{e} 089 \mathrm{e} 4$ & 3,300 & 135 & 0 & & - & - & c: program files ladobelreader 9.0 reader bib.dll \\
\hline $0 \mathrm{x} 01 \mathrm{e} 08 \mathrm{a} 44$ & 3,236 & 135 & 0 & & - & - & c: program files ladobelreader 9.0 reader bib.dll \\
\hline $0 \mathrm{x} 01 \mathrm{e} 08 \mathrm{a} 08$ & 3,232 & 134 & 0 & & - & - & c: program files ladobelreader 9.0 reader bib.dll \\
\hline $0 x 197 b 486 c$ & 3,172 & 0 & 0 & & - & - & c: program files ladobelreader 9.0 reader lagm.dll \\
\hline $0 \times 24624840$ & 2,226 & 8 & 0 & & - & - & Allocated memory or stack space \\
\hline 0x01e08ad4 & 2,082 & 151 & 0 & & - & - & c: program files ladobelreader 9.0 reader bib.dll \\
\hline $0 \mathrm{x} 19 \mathrm{a} 4 \mathrm{a} 650$ & 1,897 & 833 & 1 & 1 & - & - & c: program files ladobelreader 9.0 reader cooltype.dll \\
\hline $0 \mathrm{x} 01 \mathrm{~d} 9 \mathrm{~b} 8 \mathrm{cc}$ & 1,492 & 0 & 0 & & - & - & c: program files ladobelreader 9.0 reader sqlite.dll \\
\hline $0 \times 179 \mathrm{~d} 3 \mathrm{bc} 8$ & 1,430 & 715 & 0 & & - & - & $\begin{array}{l}\text { c:lwindows } \mid \text { winsxsix86_microsoft.vc80.crt_1fc8b3b9ale18e3b_8.0.50727.762_x-ww_6b128700 } \\
\text { msvcr80.dll }\end{array}$ \\
\hline 0x19a4afac & 1.232 & 0 & 0 & & - & - & c: program files ladobelreader 9.0 reader cooltype.dll \\
\hline one & & & & & & & 8 My Computer \\
\hline
\end{tabular}

Figure 3-14 SAPHIRE 8 v8.06.01 Thread Validator ECA Critical Sections and Waits Report 
Eile E⿱ Edit View Favorites Iools Help

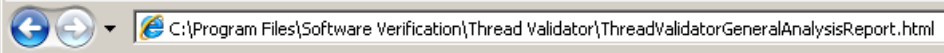

to the EAPHIRE, exe

\section{Critical Sections and Waits}

\begin{tabular}{|c|c|c|c|c|c|c|c|}
\hline numObjects:2 & \begin{tabular}{|l} 
Wait \\
Multiple
\end{tabular} & timeout:Infinite & $\begin{array}{l}0: 0 \mathrm{x} 00000 \mathrm{ae} 4 \\
1: 0 \mathrm{x} 00000 \mathrm{ad} 0\end{array}$ & $558,341 \mathrm{~ms}$ & 2088 & $\begin{array}{l}\text { StateWait } \\
\text { (UserRequest) }\end{array}$ & 0x72d2312a : midMessage \\
\hline $0 x 00159208$ & $55,706,048$ & 0 & 0 & & - & - & Allocated memory or stack space \\
\hline $0 \mathrm{x} 18 \mathrm{a} 5 \mathrm{~d} 260$ & $31,134,745$ & 1,123 & 5 & 0 & - & - & c: program files ladobelreader 9.0 readerlacrord 32 .dll \\
\hline $0 \mathrm{x} 18 \mathrm{a} 5 \mathrm{cfc} 4$ & $9,995,464$ & 0 & 0 & - & - & - & c: program files ladobelreader 9.0 |readerlacrord 32 dill \\
\hline $0 \times 18 a 5 c e b 4$ & $4,849,360$ & 0 & 0 & & - & - & c: program files ladobe reader 9.0 reader lacrord 32 .dll \\
\hline $0 \mathrm{x} 19 \mathrm{e} 4 \mathrm{dc} 38$ & $3,455,724$ & $1,477,598$ & 2 & 0 & - & - & Allocated memory or stack space \\
\hline 0x1ab3f018 & $3,005,905$ & 0 & 0 & & - & - & Allocated memory or stack space \\
\hline $0 \times 30 \operatorname{dcd} 260$ & 659,356 & 972 & 0 & & - & - & c: program filesladobelreader 9.0 readerlacrord32. dll \\
\hline $0 \mathrm{x} 3 \mathrm{dd} 2 \mathrm{dac} 0$ & 291,399 & 0 & 0 & & - & - & c:lwindows $\backslash$ system $32 \mathrm{msh}$ tml.dll \\
\hline $0 \times 30$ decfc 4 & 138,578 & 0 & 0 & - & - & - & c: program files ladobelreader 9.0 readerlacrord 32 .dll \\
\hline 0x188b8ab0 & 92,356 & 0 & 5 & 0 & - & - & c. program files ladobelreader 9.0 'readerlacrord 32 .dll \\
\hline 0x041a1738 & 62,723 & 16,087 & 0 & - & - & - & Allocated memory or stack space \\
\hline 0x188b8934 & 54,884 & 0 & 0 & & - & - & c: program files ladobelreader 9.0 readerlacrord 32 dill \\
\hline $0 \mathrm{x} 01 \mathrm{e} 08 \mathrm{ab} 0$ & 54,083 & 0 & 5 & 0 & - & - & c: program files ladobe treader 9.0 |reader bib.dll \\
\hline $0 \mathrm{x} 188 \mathrm{~b} 8 \mathrm{bfc}$ & 36,309 & 141 & 0 & & - & - & c.) program files ladobelreader 9.0 readerlacrord32. dll \\
\hline 0x1bec9c38 & 33,998 & 0 & 0 & & - & - & Allocated memory or stack space \\
\hline 0x30dcceb4 & 32,258 & 0 & 0 & & - & - & c: program filesladobelreader 9.0 readerlacrord32. dll \\
\hline $0 \mathrm{x} 01 \mathrm{e} 08934$ & 31,039 & 0 & 1 & 8 & $8-$ & - & c: program filesladobelreader 9.0 |reader bib.dll \\
\hline 0x0f244028 & 26,393 & 0 & 1 & 0 & - & - & Allocated memory or stack space \\
\hline 0x1be98208 & 23,249 & 10,774 & 0 & - & - & - & Allocated memory or stack space \\
\hline $0 \mathrm{x} 01 \mathrm{e} 08 \mathrm{bfc}$ & 20,390 & 24 & 0 & & - & - & c: program files ladobelreader 9.0 reader bib.dll \\
\hline 0x1978fc44 & 19,257 & 0 & 2 & 0 & - & - & c: program filesladobelreader 9.0 readerlagm.dll \\
\hline $0 \mathrm{x} 01 \mathrm{e} 08 \mathrm{a} 20$ & 10,385 & 0 & 0 & 0 & - & - & c: program files ladobelreader 9.0 reader bib.dll \\
\hline $0 x 188 b 8 a 20$ & 7,960 & 0 & 0 & & - & - & c: program files ladobelreader 9.0 readerlacrord32. dll \\
\hline \multirow[t]{2}{*}{ 0x19a4aeac } & 6,911 & 0 & 0 & & - & - & c: program files ladobelreader 9.0 reader cooltype.dill \\
\hline & & & & & & ! & 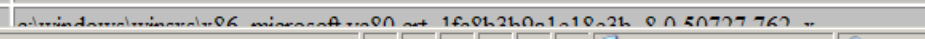 \\
\hline
\end{tabular}

Figure 3-15 SAPHIRE 8 v8.06.01 Thread Validator General Analysis Critical Sections and Waits Report 


\subsection{Bug Reporting and Trends}

This section will include a list of bugs reported by build and date, note any trends and provide recommendations.

\section{Conclusions}

We'll draw some conclusions from the latest metrics run and comparisons and provide some recommendations for future actions. 